\title{
THE INITIAL CONDITIONS FOR GRAVITATIONAL COLLAPSE OF A CORE: AN EXTREMELY YOUNG LOW-MASS CLASS 0 PROTOSTAR GF 9-2
}

\author{
RAY S. FuRUYA ${ }^{1}$ \\ Division of Physics, Mathematics, and Astronomy, California Institute of Technology, 1200 East California Boulevard \\ MS 105-24, Pasadena, CA 91125; rsf@subaru.naoj.org \\ Yoshimi Kitamura \\ Institute of Space and Astronautical Science, Japan Aerospace Exploration Agency, 3-1-1 Yoshinodai, \\ Sagamihara, Kanagawa 229-8510, Japan; kitamura@isas.jaxa.jp \\ AND \\ Hiroko ShinNaga \\ Caltech Submillimeter Observatory, California Institute of Technology, \\ 111 Nowelo Street, Hilo, HI 96720; shinnaga@submm.caltech.edu \\ Received 2005 November 22; accepted 2006 August 13
}

\begin{abstract}
We present a study of the natal core harboring the low-mass Class 0 protostar GF 9-2 in the filamentary dark cloud GF 9 using the Nobeyama $45 \mathrm{~m}$ and CSO $10.4 \mathrm{~m}$ telescopes and the OVRO millimeter array. GF 9-2 is unique in the sense that it shows $\mathrm{H}_{2} \mathrm{O}$ masers, a clear signpost of protostar formation, but does not have a high-velocity large-scale outflow. These facts indicate that the GF 9-2 core is early enough after star formation that it still retains some information of initial conditions for collapse. Our $350 \mu \mathrm{m}$ dust continuum image identified a protostellar envelope with an extent of $\simeq 5400 \mathrm{AU}$ in the center of the molecular core $\simeq 0.08 \mathrm{pc}$ in size. The envelope mass is estimated to be $\simeq 0.6 M_{\odot}$ from the $350 \mu \mathrm{m}$ flux density, while the LTE mass of the core is $\simeq 3 M_{\odot}$ from molecular lines. We found that the core has a radial density profile of $\rho(r) \propto r^{-2}$ for the $0.003 \lesssim r / \mathrm{pc} \lesssim 0.08$ region and that the velocity width of the core gas increases inward, while the outermost region maintains a velocity dispersion of a few times the ambient sound speed. If we interpret the broadened velocity width as infall, the core collapse can be well described by an extension of the Larson-Penston solution for the period after formation of a central star. We derived the mass accretion rate of $\simeq 3 \times 10^{-5} M_{\odot} \mathrm{yr}^{-1}$ from infall velocity of $\simeq 0.3 \mathrm{~km} \mathrm{~s}^{-1}$ at $r \simeq 7000 \mathrm{AU}$. Furthermore, we found evidence that a protobinary is being formed at the core center. All of the results suggest that the GF 9-2 core has been undergoing gravitational collapse for $\lesssim 5000 \mathrm{yr}$ since the protostar formation and that the unstable state initiated the collapse $\simeq 2 \times 10^{5} \mathrm{yr}$ (the free-fall time) ago.
\end{abstract}

Subject headings: ISM: clouds - ISM: evolution — ISM: individual (GF 9-2, L1082) — ISM: molecules — stars: formation - stars: pre-main-sequence

\section{INTRODUCTION}

Stars form inside dense $\left(\simeq 10^{5} \mathrm{~cm}^{-3}\right)$ and compact $(\simeq 0.1 \mathrm{pc})$ molecular cores (e.g., Myers \& Benson 1983; Mizuno et al. 1994). One of the roles of such cores is to provide material for newly forming stars through dynamical accretion caused by gravitational collapse of the cores. For isolated low-mass star formation, two extreme paradigms for collapse of a core have been proposed. One is a slow, quasi-static model, while the other extreme is the dynamic, turbulent one. The former is represented by the Shu similarity solution (Shu 1977), and the latter by the Larson-Penston solution (Larson 1969; Penston 1969), which was extended for the period after a protostar formation by Hunter (1977) and Whitworth \& Summers (1985). Hereafter we refer to the latter extended solution as the Larson-Penston solution. The two solutions are discriminated by radial density and velocity profiles of the core around $t=0$. The Shu solution (Shu 1977) sets the density profile of $\rho(r)=\left(c_{s}^{2} / 2 \pi G\right) r^{-2}$ and is static $[v(r)=0]$ at $t=0$, where $c_{s}$ and $G$ are the isothermal sound speed and the gravitational constant, respectively. At $t=0$, the collapse of the core begins at the center, which produces an expansion or rarefaction wave that propagates outward with the local sound speed. This

\footnotetext{
${ }^{1}$ Current address: Subaru Telescope, National Astronomical Observatory of Japan, 650 North A'ohoku Place, Hilo, HI 96720.
}

model is referred to as the "inside-out" collapse (Shu 1977; Shu et al. 1987). On the other hand, the Larson-Penston solution approaches the profile of $\rho(r) \simeq 4.4\left(c_{s}^{2} / 2 \pi G\right) r^{-2}$ and in infalling state $\left[v(r) \approx-3.28 c_{s}\right]$ when a protostar is about to be born $(t \rightarrow$ -0; Hunter 1977; Shu 1977; Foster \& Chevalier 1993). Thus, the Larson-Penston solution, referred to as the "runaway" collapse, predicts a 48 times higher mass infall rate than the Shu solution. At $t>0$, both solutions have a free-fall region of $\rho(r) \propto$ $r^{-3 / 2}$, which expands with sound speed for the Shu solution and with supersonic velocity for the Larson-Penston solution that accompanies no rarefaction wave.

Molecular line and dust continuum observations have identified a number of gravitationally bound starless cores (e.g., Mizuno et al. 1994), referred to as "preprotostellar" cores (WardThompson et al. 1994). Thermal dust continuum emission imaging at millimeter and submillimeter wavelengths revealed that radial density profiles of the preprotostellar cores have much flatter profiles than $\rho(r) \propto r^{-2}$ at radii smaller than a few thousand AU (e.g., Ward-Thompson et al. 1994; André et al. 1996). Molecular line studies measuring the velocity field of the cores are also powerful tools for verifying collapse models (e.g., Zhou 1992). For this purpose, it is most important to search for a starforming core that retains the initial conditions for the gravitational collapse, namely, a star-forming core at an extremely early evolutionary phase before dispersal by a large-scale molecular outflow. 
TABLE 1

Summary of Single-Dish Telescope Observations

\begin{tabular}{|c|c|c|c|c|c|c|c|c|c|}
\hline Emission & $\begin{array}{l}\text { Rest Frequency } \\
\quad(\mathrm{MHz})\end{array}$ & Telescope & Receiver & $\begin{array}{l}\theta_{\mathrm{HPBW}}{ }^{\mathrm{a}} \\
(\operatorname{arcsec})\end{array}$ & $\eta_{\mathrm{mb}}^{\mathrm{b}}$ & $\begin{array}{c}\Delta v_{\text {res }}{ }^{\mathrm{c}} \\
\left(\mathrm{km} \mathrm{s}^{-1}\right)\end{array}$ & $\begin{array}{l}\sigma_{T_{A}^{*}}{ }^{\mathrm{d}} \\
(\mathrm{mK})\end{array}$ & Map Type ${ }^{\mathrm{e}}$ & $\begin{array}{c}\text { Area }^{\mathrm{f}} \\
(\operatorname{arcmin})\end{array}$ \\
\hline $\mathrm{NH}_{3}(1,1) \ldots \ldots$ & 23694.495 & NRO & $\mathrm{H} 22$ & 78 & 0.82 & 0.477 & 46 & $\mathrm{M} / \mathrm{N}$ & $5.9 \times 5.9$ \\
\hline $\mathrm{NH}_{3}(3,3) \ldots \ldots \ldots \ldots \ldots \ldots \ldots$ & 23870.130 & NRO & $\mathrm{H} 22$ & 78 & 0.82 & 0.473 & 58 & $\mathrm{M} / \mathrm{N}$ & $5.9 \times 5.9$ \\
\hline $\mathrm{NH}_{3}(4,4) \ldots \ldots \ldots$ & 24139.417 & NRO & $\mathrm{H} 22$ & 78 & 0.82 & 0.469 & 62 & $\mathrm{M} / \mathrm{N}$ & $5.9 \times 5.9$ \\
\hline $\mathrm{CC}^{34} \mathrm{~S}_{4}-3_{2} \ldots \ldots \ldots \ldots$ & 44497.559 & NRO & $\mathrm{S} 40$ & 40 & 0.76 & 0.253 & 25 & $\mathrm{D}$ & $\ldots$ \\
\hline $\mathrm{C}_{3} \mathrm{H}_{2} 2_{12}-1_{01} \ldots \ldots \ldots \ldots \ldots \ldots \ldots$ & 85338.906 & NRO & S100 & 18.7 & 0.50 & 0.130 & 30 & $\mathrm{D}$ & $\ldots$ \\
\hline \multirow[t]{2}{*}{$\mathrm{H}^{13} \mathrm{CO}^{+}(1-0) \ldots \ldots \ldots \ldots \ldots \ldots$} & 86754.330 & NRO & $\mathrm{S} 80 / \mathrm{S} 100$ & 18.5 & 0.50 & 0.128 & 37 & $\mathrm{C} / \mathrm{F}$ & $3.7 \times 2.4$ \\
\hline & 86754.330 & NRO & BEARS & 20.3 & 0.49 & 0.108 & 48 & $\mathrm{M} / \mathrm{F}$ & $8.6 \times 8.6$ \\
\hline \multirow[t]{2}{*}{$\mathrm{SiO}(1-0) v=0 \ldots \ldots \ldots \ldots . . .}$. & 86846.998 & NRO & S80 & 18.5 & 0.50 & 0.128 & 42 & $\mathrm{M} / \mathrm{F}$ & $3.7 \times 2.4$ \\
\hline & 86846.998 & NRO & BEARS & 20.5 & 0.49 & 0.108 & 185 & $\mathrm{M} / \mathrm{F}$ & $8.6 \times 8.6$ \\
\hline $\mathrm{N}_{2} \mathrm{H}^{+}(1-0)$ & 93173.900 & NRO & $\mathrm{S} 80 / \mathrm{S} 100$ & 17.2 & 0.51 & 0.119 & 31 & $\mathrm{M} / \mathrm{F}$ & $2.0 \times 2.0$ \\
\hline${ }^{12} \mathrm{CO}(1-0) \ldots \ldots \ldots \ldots$ & 115271.204 & NRO & $\mathrm{S} 100$ & 14.8 & 0.49 & 0.0976 & 94 & $\mathrm{C} / \mathrm{F}$ & $2.8 \times 1.8$ \\
\hline
\end{tabular}

${ }^{\text {a }}$ Half-power beamwidth for a circular Gaussian beam.

${ }^{b}$ Main-beam efficiency.

c Effective velocity resolution.

d Typical rms noise level of the spectrum. For detection observations, an rms noise level of the spectrum is given. For mapping observations, a mean rms noise level for all of the spectra is given.

e Observing modes for the molecular line emission; D denotes "detection" observations toward the center position of R.A. $=20^{\mathrm{h}} 51^{\mathrm{m}} 22^{\mathrm{s}} .5$, decl. $=60^{\circ} 18^{\prime} 38^{\prime \prime} 0$ (J2000.0), M/F and M/N denote, respectively, "mapping" observations with full-beam and Nyquist-sampled grid spacings centered on the above position, and C/F denotes "cross scan" observations with a full-beam spacing along the northeast-southwest and northwest-southeast directions centered on the position.

${ }^{\mathrm{f}}$ Size of the region for the mapping observations, and the major- and minor-axis lengths for cross scan ones.

Furuya et al. $(1999,2000)$ proposed that the intermediate-mass Class 0 source S106 FIR is such an ideal core because the source is characterized by (1) a cold spectral energy distribution (SED; Richer et al. 1993); (2) $\mathrm{H}_{2} \mathrm{O}$ maser emission, i.e., strong evidence for the presence of a protostar; and (3) the absence of a large-scale high-velocity $\mathrm{CO}$ outflow.

We have successfully identified a low-mass counterpart, GF 9-2, by performing a multiepoch $\mathrm{H}_{2} \mathrm{O}$ maser survey (Furuya et al. 2001,2003 ) and ${ }^{12} \mathrm{CO}$ outflow mapping survey toward the selected low-mass protostars with the Nobeyama $45 \mathrm{~m}$, SEST $15 \mathrm{~m}$, and CSO $10.4 \mathrm{~m}$ telescopes (R. S. Furuya et al. 2007, in preparation). In our sample, GF 9-2 is the only protostar satisfying characteristics 1-3 above. This protostar is located in the east-west filamentary dark cloud GF 9 where several YSOs are located every $\simeq 0.75 \mathrm{pc}$ in the plane of the sky (Schneider \& Elmegreen 1979; Wiesemeyer 1997); the filament is cross identified as Barnard 150 and Lynds 1082. The distance to GF 9-2 is estimated to be 200 pc by Wiesemeyer (1997). Wiesemeyer (1997) and Wiesemeyer et al. (1999) identified GF 9-2 as Class 0 and obtained a bolometric temperature $\left(T_{\text {bol }}\right)$ of $\leq 20 \mathrm{~K}$ and bolometric luminosity $\left(L_{\text {bol }}\right)$ of $0.3 L_{\odot}$. We believe that GF 9-2 offers a unique opportunity to investigate the initial conditions for gravitational collapse of a star-forming core. This paper presents a detailed observational study of GF 9-2. In particular, we combined millimeter interferometer and single-dish telescope visibility data to obtain high-fidelity images of the core.

\section{OBSERVATIONS}

Toward GF 9-2, we observed dust continuum emission with the Caltech Submillimeter Observatory ${ }^{2}$ (CSO) 10.4 m telescope

\footnotetext{
2 The Caltech Submillimeter Observatory is operated by the California Institute of Technology under funding from the US National Science Foundation (AST 02-29008).
}

(§ 2.1) and molecular line emission with the Nobeyama Radio Observatory $^{3}$ (NRO) 45 m telescope $(\S 2.2)$. We also carried out a deep search for molecular outflow using the 10.4 and $45 \mathrm{~m}$ telescopes and used centimeter radio continuum data from the $\mathrm{NRAO}^{4}$ Very Large Array (VLA) archive database to search for a thermal radio jet $(\$ 2.4)$. The parameters for single-dish and interferometer observations are summarized in Tables 1 and 2, respectively.

\subsection{Caltech Submillimeter Observatory 10.4 m Telescope}

\subsubsection{Mapping of ${ }^{12} \mathrm{CO}(3-2)$ Line Emission}

The ${ }^{12} \mathrm{CO}(3-2)$ line observations were done using the SIS receiver with position switching mode in 2002 December. We used the $50 \mathrm{MHz}$ bandwidth acousto-optical spectrometer (AOS). During the observations, the zenith opacities at $225 \mathrm{GHz}\left(\tau_{225 \mathrm{GHz}}\right)$ ranged from 0.037 to 0.12 . The telescope pointing was checked by observing the ${ }^{12} \mathrm{CO}(3-2)$ emission toward CRL 2688 every $1.5 \mathrm{hr}$, and the accuracy was better than $5^{\prime \prime}$. The observing grid was centered on the position of the $3 \mathrm{~mm}$ continuum source reported in Wiesemeyer et al. (1999) (R.A. $=20^{\mathrm{h}} 51^{\mathrm{m}} 29^{\prime \prime} .5$, decl. $=$ $60^{\circ} 18^{\prime} 38^{\prime \prime}$. [J2000.0]). In the data reduction, we removed spectra obtained with an elevation angle of $\lesssim 30^{\circ}$ or with $T_{\text {sys }} \geq 800 \mathrm{~K}$ in a single sideband (SSB).

\subsubsection{Imaging of $350 \mu \mathrm{m}$ Continuum Emission with SHARC II}

The SHARC II camera (Dowell et al. 2003) was used to obtain a $350 \mu \mathrm{m}$ continuum emission map in the box and Lissajous

\footnotetext{
3 Nobeyama Radio Observatory is a branch of the National Astronomical Observatory, operated by the Ministry of Education, Culture, Sports, Science and Technology, Japan.

4 The National Radio Astronomy Observatory is a facility of the National Science Foundation operated under cooperative agreement by Associated Universities, Inc.
} 
TABLE 2

Summary of InTERferometric ObServations

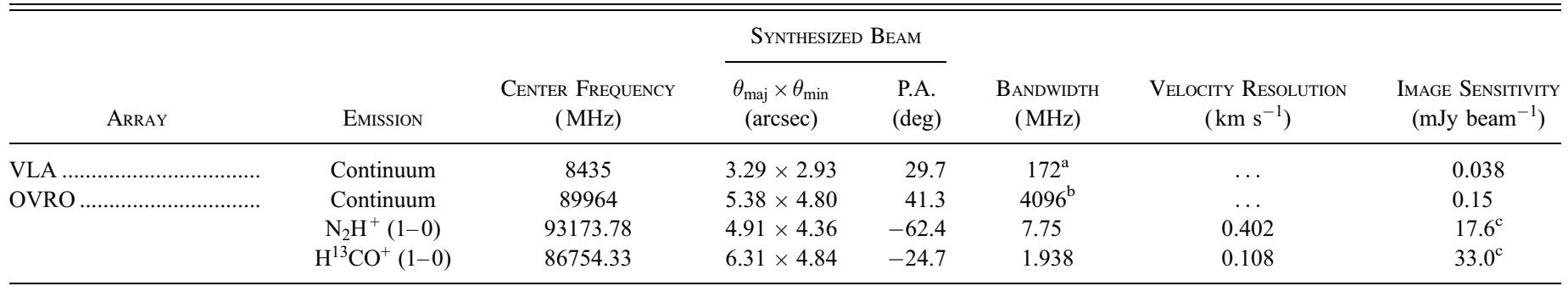

${ }^{\text {a }}$ Concatenated the two polarization data.

b Concatenated the lower and upper sideband data.

${ }^{\mathrm{c}}$ Per velocity channel.

scan modes (Kovács 2006) in 2004 June. We used the Dish Surface Optimization System (DSOS), which actively corrects gravitational deformation of the main reflector of the telescope (Leong 2005). During our observations, $\tau_{225 \mathrm{GHz}}$ ranged from 0.045 to 0.08 . The telescope focusing and pointing were checked every hour by observing Neptune and Uranus. The measured beam size in half-power beamwidth (HPBW) was $\left(8^{\prime \prime} .9 \pm 0.2\right) \times\left(8^{\prime \prime} .0 \pm\right.$ $0.4)$ at P.A. $=93^{\circ}$. We estimated the positional accuracy of $\lesssim 4^{\prime \prime}$ from maps of the pointing sources. For flux calibrations, we used the pointing data of Neptune that satisfied the condition of $\tau_{225 \mathrm{GHz}} \times$ air mass $\leq 0.1$. We obtained a mean peak voltage $\left(V_{\text {peak }}\right)$ of $8.25 \pm 1.5 \mu \mathrm{V}$ toward Neptune over a pixel aperture $\left(A_{\text {pixel }}=4 " .9 \times 4 . " 8\right)$ of the detector. The measured voltage was converted into source intensity as $V_{\text {peak }} / A_{\text {pixel }} \propto S_{\nu} / \Omega_{b}$, where $S_{\nu}$ denotes flux density per beam and $\Omega_{b}$ beam solid angle. Since the brightness temperature of $63 \mathrm{~K}$ and the angular diameter of 2.6 for Neptune led to $S_{\nu}$ of $98.0 \mathrm{Jy}$ at $350 \mu \mathrm{m}$, we obtained a conversion factor of $3.2 \times 10^{-6} \mathrm{Jy} \mathrm{beam}^{-1} \mathrm{~V}^{-1}$.

\subsection{Nobeyama $45 \mathrm{~m}$ Telescope \\ 2.2.1. Observations of ${ }^{12} \mathrm{CO}(1-0), \mathrm{H}^{13} \mathrm{CO}^{+}, \mathrm{HC}^{18} \mathrm{O}^{+}$ (1-0), and $\mathrm{C}_{3} \mathrm{H}_{2} 2{ }_{12}-1_{01}$ Lines}

We carried out simultaneous observations of ${ }^{12} \mathrm{CO}(1-0)$ and $\mathrm{H}^{13} \mathrm{CO}^{+}(1-0)$ lines using two SIS receivers (S100 and S80) with SSB filters in dual polarization mode in 2000 April. For the back end, we used the eight arrays of AOS. All of the $45 \mathrm{~m}$ telescope observations were made in position switching mode, using an off position of $(\Delta \alpha, \Delta \delta)=\left(-8{ }^{\prime} 0,+10^{\prime} 0\right)$ to the $3 \mathrm{~mm}$ source. The pointing accuracy was better than $3^{\prime \prime}$. The daily variation of the receiver gains was estimated to be less than $10 \%$ in peak to peak, leading to the corrected absolute flux uncertainty of $15 \%$. In 2005 April, we made the simultaneous observations of the $\mathrm{H}^{13} \mathrm{CO}^{+}$and $\mathrm{HC}^{18} \mathrm{O}^{+}(1-0)$ lines to measure optical depth of the $\mathrm{H}^{13} \mathrm{CO}^{+}$line toward the $3 \mathrm{~mm}$ continuum source. At the same time, we also observed the ortho- $\mathrm{C}_{3} \mathrm{H}_{2} 2_{12}-1_{01}$ line.

\subsubsection{Mapping Observations of ${ }^{12} \mathrm{CO}(1-0), \mathrm{H}^{13} \mathrm{CO}^{+}(1-0)$, and $\mathrm{SiO}(2-1)$ Lines with BEARS}

The mapping observations of the ${ }^{12} \mathrm{CO}(1-0), \mathrm{H}^{13} \mathrm{CO}^{+}(1-0)$, and $\mathrm{SiO}(2-1) v=0$ lines were carried out with the 25 Beam Array Receiver System (BEARS) in 2001 January and April. For back end, we used digital spectrometers. We adopted correction factors provided by the observatory for relative gain differences among the 25 beams. The ${ }^{12} \mathrm{CO}$ and $\mathrm{H}^{13} \mathrm{CO}^{+}$line data taken with the S100/S80 receivers with the SSB filters ( $\S 2.2 .1)$ were used to determine the antenna temperature $\left(T_{A}^{*}\right)$ scale for the BEARS, which is not equipped with SSB filters. The tele- scope pointing accuracy was better than $3^{\prime \prime}$, and the uncertainty of temperature scale calibration was better than $20 \%$.

$$
\begin{aligned}
& \text { 2.2.3. Observations of } \mathrm{N}_{2} \mathrm{H}^{+}(1-0) \text {, CCS } 4_{3}-3_{2} \text {, } \\
& \text { and } \mathrm{CC}^{34} \mathrm{~S}_{3}-3_{2} \text { Lines }
\end{aligned}
$$

In 2003 December, we made the simultaneous $\mathrm{N}_{2} \mathrm{H}^{+}(1-0)$ and CCS $44_{3}-3_{2}$ line observations with a grid spacing of $40^{\prime \prime}$, fullbeam sampling for the CCS line. Subsequently, we carried out deeper integration of the $\mathrm{N}_{2} \mathrm{H}^{+}$line with a spacing of $20^{\prime \prime}$, a fullbeam sampling for the line. In addition, we observed the $\mathrm{CC}^{34} \mathrm{~S}$ $4_{3}-3_{2}$ line toward the $3 \mathrm{~mm}$ continuum source to estimate the optical depth of the CCS line. The pointing accuracy and the uncertainty of flux calibration were the same as in $\S 2.2 .2$.

\subsubsection{Observations of $\mathrm{NH}_{3}$ Inversion Lines at $23 \mathrm{GHz}$}

We carried out mapping observations of the four inversion lines of $\mathrm{NH}_{3}$ molecules at 23-24 GHz using the HEMT receiver (H20) with a dual circular polarization mode in 2001 April. We used the eight AOS arrays to receive both of the polarizations of the four transitions and added the dual polarization data for each transition to improve the signal-to-noise ratio $(\mathrm{S} / \mathrm{N})$. The telescope pointing accuracy was better than $5^{\prime \prime}$, and the uncertainty of temperature calibration was better than $20 \%$.

\subsection{Owens Valley Radio Observatory Millimeter Array}

The aperture synthesis observations at $3 \mathrm{~mm}$ were carried out using the six-element Owens Valley Radio Observatory (OVRO) millimeter array in the period from 2003 September to 2004 May with the four array configurations: three full tracks at the $\mathrm{C}$ configuration, five at $\mathrm{L}$, two at $\mathrm{H}$, and two partial tracks at $\mathrm{E}$ (Table 2). The resultant projected baseline length ranged from 12.8 to $224 \mathrm{~m}$; our OVRO observations are insensitive to structures extended more than $54^{\prime \prime}$, corresponding to $0.052 \mathrm{pc}$ at $d=200 \mathrm{pc}$. We used the $3 \mathrm{~mm}$ continuum source position reported in Wiesemeyer et al. (1999) as the phase tracking center. We observed the $\mathrm{N}_{2} \mathrm{H}^{+}(1-0)$ line in the upper sideband and the $\mathrm{H}^{13} \mathrm{CO}^{+}(1-0)$ line in the lower sideband. The velocity resolutions were 0.402 and $0.108 \mathrm{~km} \mathrm{~s}^{-1}$ for the $\mathrm{N}_{2} \mathrm{H}^{+}$and $\mathrm{H}^{13} \mathrm{CO}^{+}$lines, respectively.

We used 3C 273, 3C 454.3, and 3C 84 as passband calibrators and $\mathrm{J} 2038+513$ as a phase and gain calibrator. The flux densities of J2038+513 were determined from observations of Uranus and Neptune. The measured flux densities were fairly stable in the range of $1.37-1.43 \mathrm{Jy}$ at $86.7 \mathrm{GHz}$ and of $1.26-1.38 \mathrm{Jy}$ at $93.2 \mathrm{GHz}$. The uncertainty in our flux calibration was estimated to be $10 \%$. To minimize atmospheric phase fluctuation errors, we removed all of the GF 9-2 data taken in the switching cycles when the coherency of the calibrator was less than $70 \%$. The data were calibrated and edited using the MMA and MIRIAD packages. For the 
TABLE 3

Summary of the Combined Data from the OVRO Millimeter Array and the NRO $45 \mathrm{~m}$ Telescope

\begin{tabular}{|c|c|c|c|c|c|c|}
\hline \multirow[b]{3}{*}{ LiNE } & \multirow{2}{*}{\multicolumn{2}{|c|}{ Spatial Frequency Range $(\mathrm{k} \lambda)$}} & \multirow{3}{*}{$\begin{array}{l}\text { FIELD OF VIEW } \\
\quad(\operatorname{arcsec})\end{array}$} & \multicolumn{2}{|c|}{ Synthesized Beam } & \multirow{3}{*}{$\begin{array}{l}\text { Veloctit Resolution } \\
\qquad\left(\mathrm{km} \mathrm{s}^{-1}\right)\end{array}$} \\
\hline & & & & \multirow{2}{*}{$\begin{array}{c}\theta_{\mathrm{maj}} \times \theta_{\mathrm{min}} \\
\quad(\operatorname{arcsec})\end{array}$} & \multirow{2}{*}{$\begin{array}{l}\text { P.A. } \\
\text { (deg) }\end{array}$} & \\
\hline & $45 \mathrm{~m}$ & OVRO & & & & \\
\hline $\mathrm{N}_{2} \mathrm{H}^{+}(1-0) \ldots \ldots \ldots \ldots \ldots \ldots$ & $0.0-5.29$ & $3.30-71.8$ & 69.7 & $4.49 \times 3.86$ & -70.7 & 0.402 \\
\hline $\mathrm{H}^{13} \mathrm{CO}^{+}(1-0) \ldots \ldots \ldots \ldots \ldots$ & $0.0-4.48$ & $3.07-58.0$ & 75.0 & $6.73 \times 5.14$ & -27.2 & 0.108 \\
\hline
\end{tabular}

Note.-For details, see Appendix A.

continuum data, we merged the visibilities in both of the sidebands to construct an image; the final center frequency is $89.964 \mathrm{GHz}$. Images of both the line and continuum were constructed using the AIPS package.

Since an interferometer does not have sensitivity to structures extended more than the largest fringe spacing given by the shortest projected baseline length, we used the $45 \mathrm{~m}$ telescope maps to recover filtered-out emission in our OVRO millimeter array observations (Table 3; Appendix A). After combining interferometer and single-dish visibilities, we CLEANed maps using the task IMAGR in the AIPS package. The parameters of the final maps are given in Table 3 .

\subsection{Very Large Array}

We used the $8435 \mathrm{MHz}$ continuum emission image of GF 9-2 from the VLA archive database. The data were originally taken by Wiesemeyer et al. (1999) in 1997 July with the C configuration (Table 2). The flux densities of calibrators of J2022+616 and $\mathrm{J} 2055+613$ were determined from observations of the standard calibration sources of 3C 286 and 3C 48, and the uncertainty in the final flux calibration was better than $20 \%$.

\section{RESULTS}

\subsection{Continuum Emission}

\subsubsection{Nondetection of Free-Free Emission at $8.4 \mathrm{GHz}$}

No radio continuum emission from ionized gas is detected toward GF 9-2 through our VLA C configuration search at $8.4 \mathrm{GHz}$ $(\lambda=3.6 \mathrm{~cm})$. The attained $3 \sigma$ sensitivity is $0.11 \mathrm{mJy} \mathrm{beam}^{-1}$, leading to the upper limit of the $3.6 \mathrm{~cm}$ continuum luminosity of $0.005 \mathrm{mJy} \mathrm{kpc}{ }^{2}$ within the beam. This upper limit is lower than the luminosity of the least luminous thermal centimeter continuum emission (see Fig. 7 of Cabrit \& Bertout 1992). Since centimeter continuum emission toward protostars is usually attributed to free-free emission from ionized gas produced by strong shocks of protostellar jets, the lack of the centimeter emission in GF 9-2 suggests that a jet, if it exists, is not powerful enough to ionize ambient gas.

\subsubsection{Thermal Dust Emission at $3 \mathrm{~mm}$ and $350 \mu \mathrm{m}$}

In Figure 1 we present a comparison between the continuum emission maps at $350 \mu \mathrm{m}$ taken with the SHARC II camera and those at $3 \mathrm{~mm}$ with the OVRO millimeter array. The maps show that a $3 \mathrm{~mm}$ point source is embedded in the extended $350 \mu \mathrm{m}$ emission. The peak position of the $3 \mathrm{~mm}$ emission is R.A. $=$ $20^{\mathrm{h}} 51^{\mathrm{m}} 29^{\mathrm{s}} .827$, decl. $=60^{\circ} 18^{\prime} 38^{\prime \prime} .06$ in $\mathrm{J} 2000.0$. The $350 \mu \mathrm{m}$ emission has a size of $33^{\prime \prime} .0 \times 22$ ". 5 , corresponding to $6600 \times$ $4500 \mathrm{AU}$, which is comparable to the typical size of a protostellar envelope ( $\leq 6000 \mathrm{AU}$; Mundy et al. 2000). It is most likely that the $350 \mu \mathrm{m}$ emission traces a protostellar envelope, whereas the $3 \mathrm{~mm}$ emission is more compact, perhaps a protoplanetary disk. We assume that a protostar is located at the peak of the $3 \mathrm{~mm}$ emission.
By integrating the emission inside the $3 \sigma$ contours, we obtain the total flux densities $S_{\nu}$ (Table 4). Here we consider that the $3 \mathrm{~mm}$ emission has negligible contribution from optically thin freefee emission because our nondetection of the $8.4 \mathrm{GHz}$ continuum emission gives a $3 \sigma$ upper limit of $0.090 \mathrm{mJy}$ at $3 \mathrm{~mm}$ for the free-free emission by extrapolating with $I_{\nu} \propto \nu^{-0.1}$.

Assuming that the dust continuum emission is optically thin, we estimate the envelope mass $\left(M_{\text {env }}\right)$ from the $350 \mu \mathrm{m}$ total flux density. We used an equation of $M_{\text {env }}=S_{\nu} d^{2} / \kappa_{\nu} B_{\nu}\left(T_{d}\right)$, where $\kappa_{\nu}$ is the dust mass opacity coefficient, $T_{d}$ is the dust temperature, and $B_{\nu}\left(T_{d}\right)$ is the Planck function. Assuming that dust and gas are well coupled, we adopt $T_{d}$ of $9.5 \mathrm{~K}$, a mean excitation temperature $\left(T_{\text {ex }}\right)$ obtained from the $\mathrm{N}_{2} \mathrm{H}^{+}(1-0)$ data (described in $\left.\S 4.1 .1\right)$. The value of $\kappa_{\nu}$ at $350 \mu \mathrm{m}$ is estimated by the usual form of $\kappa_{\nu}=$ $\kappa_{\nu 0}\left(\nu / \nu_{0}\right)^{\beta}$. Here we used two sets of the representative $\kappa_{0}$ and $\beta$ values reported in preprotostellar cores (case I) and in envelopes around Class $0 /$ I protostars (case II) because $\kappa_{0}$ is known to change with evolutionary class. Recall that the source is very likely to be in an evolutionary phase between a preprotostellar core and a Class 0 protostar. Given $\kappa_{0}$ of $0.005 \mathrm{~cm}^{2} \mathrm{~g}^{-1}$ at $\nu_{0}=231 \mathrm{GHz}$ (Preibisch et al. 1993; André et al. 1996) and $\beta$ of 2.0 (Beckwith et al. 2000

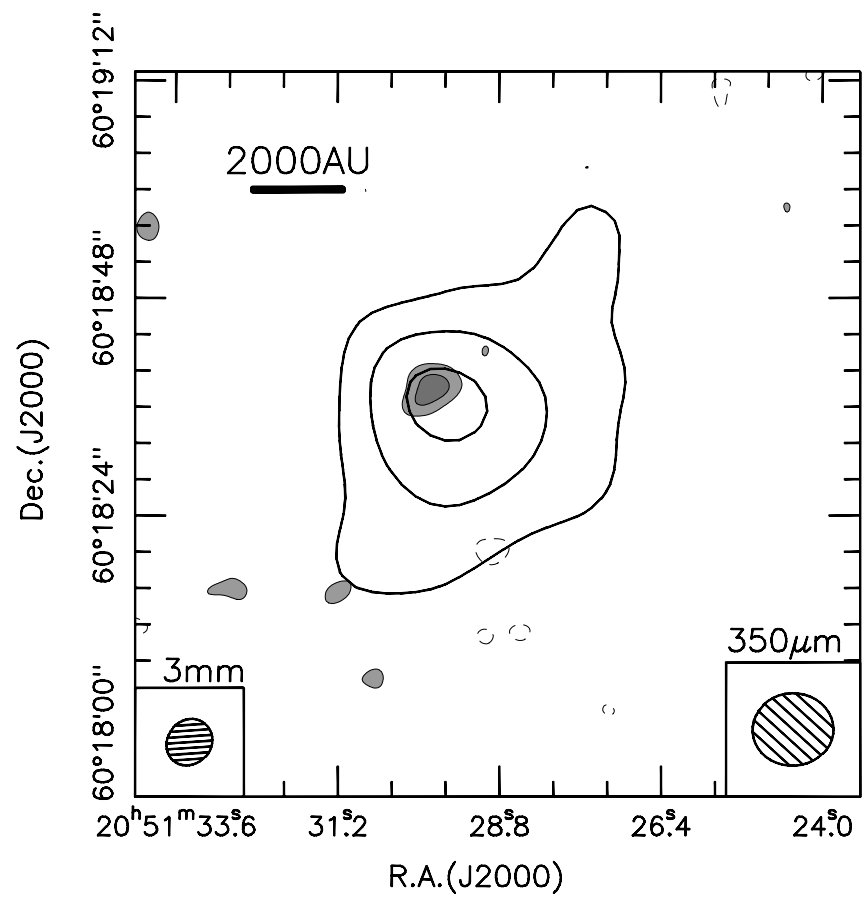

FIG. 1.-Overlay of the $3 \mathrm{~mm}$ continuum emission map observed with the OVRO millimeter array ( gray scale plus contours) on the $350 \mu \mathrm{m}$ map with the CSO SHARC II camera (thick contours). The contours start from the $3 \sigma$ levels with a $3 \sigma$ interval where $\sigma=0.15$ and $29.7 \mathrm{mJy} \mathrm{beam}^{-1}$ for the $3 \mathrm{~mm}$ and $350 \mu \mathrm{m}$ images, respectively. The dashed contours show the $-3 \sigma$ level for the $3 \mathrm{~mm}$ image. The $350 \mu \mathrm{m}$ image does not have negative contours below the $-2.2 \sigma$ level. The ellipses at the bottom corners indicate the beam sizes. 
TABLE 4

Results of Continuum Emission Flux Measurements

\begin{tabular}{|c|c|c|c|c|c|}
\hline \multirow[b]{2}{*}{$\begin{array}{c}\lambda \\
(\mu \mathrm{m})\end{array}$} & \multirow[b]{2}{*}{ INSTRUMENTS } & \multicolumn{2}{|l|}{ Size } & \multirow[b]{2}{*}{$\begin{array}{l}\text { Peak InTENsity } \\
\left(\mathrm{mJy} \mathrm{beam}^{-1}\right)\end{array}$} & \multirow[b]{2}{*}{$\begin{array}{c}\text { Total Flux Density } \\
(\mathrm{m} \mathrm{Jy})\end{array}$} \\
\hline & & $\begin{array}{l}l_{\mathrm{maj}} \times l_{\min } \\
(\operatorname{arcsec})\end{array}$ & $\begin{array}{l}\text { P.A. } \\
\text { (deg) }\end{array}$ & & \\
\hline $35540 \ldots \ldots \ldots \ldots \ldots \ldots$ & VLA D & & & $<0.055$ & \\
\hline $3330 \ldots \ldots \ldots \ldots \ldots \ldots \ldots .$. & OVRO & $(6.1 \pm 0.9) \times(4.3 \pm 0.6)$ & $129 \pm 17$ & $1.16 \pm 0.17$ & $0.75 \pm 0.07$ \\
\hline $350 \ldots \ldots \ldots \ldots \ldots \ldots$ & CSO SHARC II & $(32.9 \pm 4.3) \times(22.5 \pm 2.9)$ & $140 \pm 13$ & $300 \pm 30$ & $1830 \pm 330$ \\
\hline
\end{tabular}

and references therein) for case I, we have $\kappa$ of $0.069 \mathrm{~cm}^{2} \mathrm{~g}^{-1}$ at $850 \mathrm{GHz}$. On the other hand, given $\kappa_{0}$ of $0.01 \mathrm{~cm}^{2} \mathrm{~g}^{-1}$ at $231 \mathrm{GHz}$ (Ossenkopf \& Henning 1994) and $\beta$ of 1.0 (Beckwith et al. 2000) for case II, we have $\kappa$ of $0.037 \mathrm{~cm}^{2} \mathrm{~g}^{-1}$ at $850 \mathrm{GHz}$. Finally, we obtain the total masses of $0.4 M_{\odot}$ for case I and $0.8 M_{\odot}$ for case II. The inferred mass range is comparable to the least massive envelope masses in Class 0 sources known to date, i.e., $M_{\text {env }} \simeq 0.5 M_{\odot}$ for HH 25 MMS-MM and L1157 MM (See Table 1 of André et al. $2000)$. Using the source size $\left(\Omega_{s}\right)$ of $957 \operatorname{arcsec}^{2}$ inside the $3 \sigma$ level contour and $M_{\text {env }}$, a mean column density of $\mathrm{H}_{2}$ molecules, $N\left(\mathrm{H}_{2}\right)_{8^{\prime \prime}}$, is estimated to be $4.2 \times 10^{22}$ and $7.8 \times 10^{22} \mathrm{~cm}^{-2}$ for cases I and II, respectively. These are converted into a mean optical depth of $\simeq 7 \times 10^{-3}$ for both cases, justifying our assumption of optically thin emission.

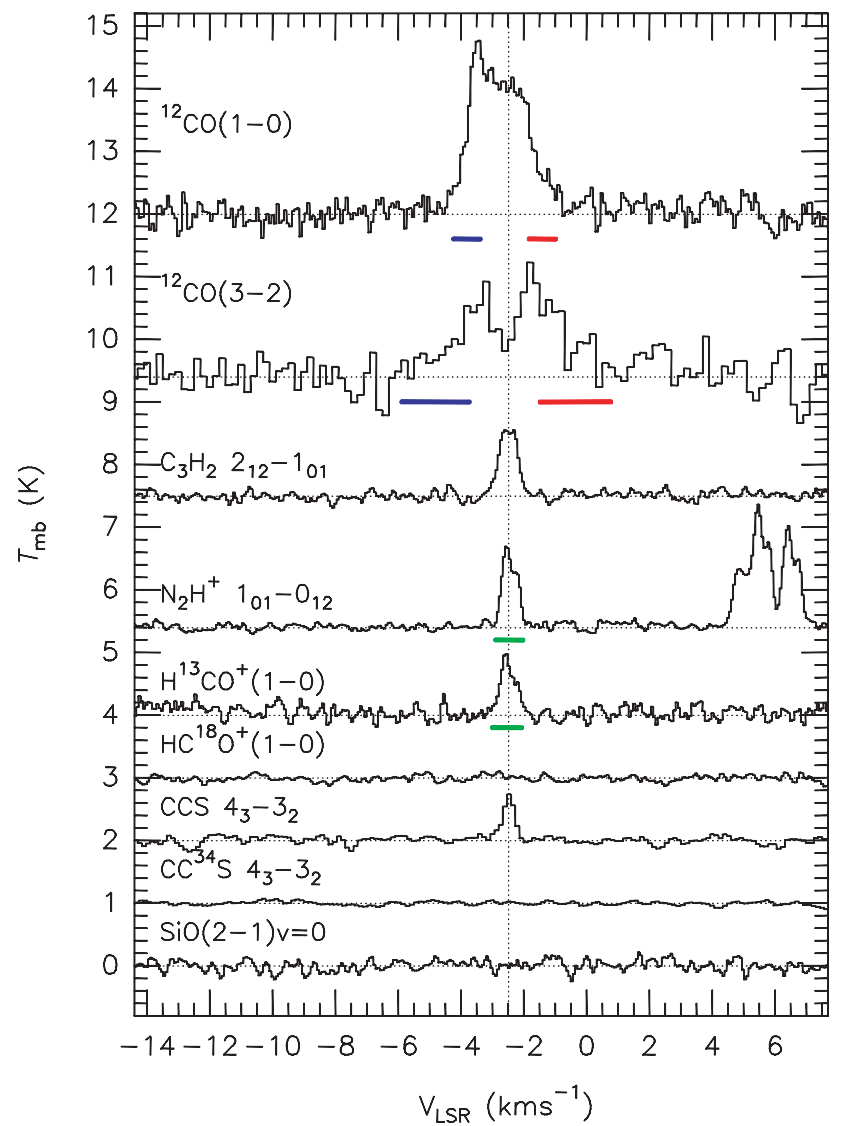

FIG. 2.-Spectra of the molecular line emission shown in the main-beam brightness temperature $\left(T_{\mathrm{mb}}\right)$ scale toward the $3 \mathrm{~mm}$ source. The vertical dashed line indicates the systemic velocity $\left(V_{\text {sys }}\right)$ of $-2.5 \mathrm{~km} \mathrm{~s}^{-1}(\S 3.2 .1)$. The horizontal blue and red bars under the ${ }^{12} \mathrm{CO}$ lines indicate the LSR velocity ranges for the integrated intensity maps shown in Fig. 8, and the green bars under the $\mathrm{N}_{2} \mathrm{H}^{+}$ and $\mathrm{H}^{13} \mathrm{CO}^{+}$lines are for the maps shown in Fig. 6. The $\mathrm{N}_{2} \mathrm{H}^{+}$emission seen in $4.5 \lesssim V_{\mathrm{LSR}} / \mathrm{km} \mathrm{s}^{-1} \lesssim 7.2$ is the main HF components (see Fig. 3).

\subsection{Molecular Line Emission}

\subsubsection{Spectral Profile of the Molecular Line Emission toward the Center}

In Figure 2 we present the spectra of ${ }^{12} \mathrm{CO}(1-0),(3-2), \mathrm{N}_{2} \mathrm{H}^{+}$ $(1-0)\left(F_{1}, F\right)=(0,1)-(1,2), \mathrm{H}^{13} \mathrm{CO}^{+}, \mathrm{HC}^{18} \mathrm{O}^{+}(1-0), \mathrm{C}_{3} \mathrm{H}_{2}$ $2_{12}-1_{01}, \mathrm{CCS}, \mathrm{CC}^{34} \mathrm{~S}_{3}-3_{2}$, and $\mathrm{SiO}(2-1) v=0$ lines toward the $3 \mathrm{~mm}$ continuum source. Figure 3 represents the $\mathrm{N}_{2} \mathrm{H}^{+}(1-0)$ spectrum to show all of the hyperfine (HF) components. We summarize the parameters of these spectra in Table 5 . Intrinsic velocity width $\left(\Delta v_{\text {int }}\right)$ in the table indicates the velocity width corrected for line broadening (where appropriate) due to instrumental velocity resolution and optical depth of the line (Phillips et al. 1979). The ${ }^{12} \mathrm{CO}(1-0)$ spectrum shows a broad line width of $\simeq 2 \mathrm{~km} \mathrm{~s}^{-1}$ in FWHM. The ${ }^{12} \mathrm{CO}(3-2)$ spectrum displays a blueshifted wing emission up to $V_{\mathrm{LSR}} \simeq-6 \mathrm{~km} \mathrm{~s}^{-1}$ and a less prominent red wing up to $\simeq 0.5 \mathrm{~km} \mathrm{~s}^{-1}$. The spectrum has a central dip at $V_{\mathrm{LSR}}=$ $-2.8 \mathrm{~km} \mathrm{~s}^{-1}$, while the high-density gas tracers of $\mathrm{N}_{2} \mathrm{H}^{+}, \mathrm{H}^{13} \mathrm{CO}^{+}$, $\mathrm{C}_{3} \mathrm{H}_{2}$, and CCS show their peaks around the dip of the ${ }^{12} \mathrm{CO}(3-2)$ spectrum. Such a coincidence indicates that the two peaks in the ${ }^{12} \mathrm{CO}(3-2)$ spectrum arise from self-absorption, and not from two velocity components along the line of sight. At ${ }^{12} \mathrm{CO}(1-0)$, the self-absorption is broad; the peak in the redshifted side is almost missing, and only the blueshifted peak is seen. Here we define systemic velocity $\left(V_{\text {sys }}\right)$ of the cloud core as $-2.5 \mathrm{~km} \mathrm{~s}^{-1}$, the peak velocity of the CCS $4_{3}-3_{2}$ line, because the line has a single sharp peak and is optically thin (described in $\S 4.1 .4$ ). In addition, the $\mathrm{H}^{13} \mathrm{CO}^{+}$and $\mathrm{N}_{2} \mathrm{H}^{+}$lines show similar line profiles in the sense that

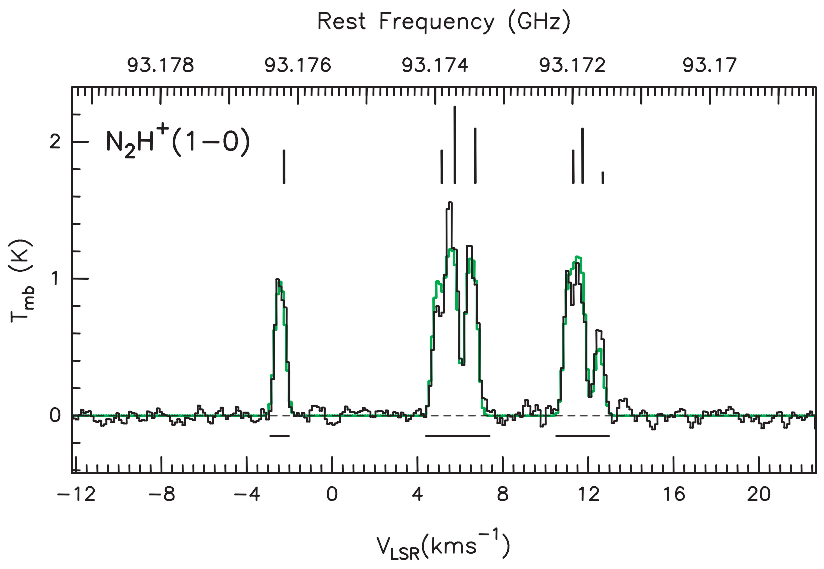

FIG. 3. $-\mathrm{N}_{2} \mathrm{H}^{+}(1-0)$ spectrum (black histogram) taken with the $45 \mathrm{~m}$ telescope toward the $3 \mathrm{~mm}$ source. The green histogram represents the best-fit spectrum from the $\mathrm{HF}$ analysis ( $(4.1 .1)$. We assume that the LSR velocity of the isolated $\left(F_{1}, F\right)=(0,1)-(1,2)$ transition $\left(\nu_{\text {rest }}=93176.310 \mathrm{MHz}\right)$ is equal to the $V_{\text {sys }}$ of $-2.5 \mathrm{~km} \mathrm{~s}^{-1}$. The lengths of vertical lines above the spectrum indicate the expected relative intensities at $\tau \simeq 0$, plotted at the rest frequencies (Cazolli et al. 1985). The horizontal bars below the spectrum show the velocity ranges integrated to obtain maps in Fig. $4 c$ and those in the first row of Fig. 6. 
TABLE 5

Results from the 45 m Telescope Spectral Line Observations: Dense Gas Tracers

\begin{tabular}{|c|c|c|c|c|c|c|}
\hline \multirow[b]{2}{*}{ LiNE } & \multicolumn{3}{|c|}{ 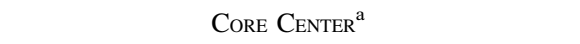 } & \multicolumn{3}{|c|}{ Inside the Half-MaXimum Contour $^{\text {b }}$} \\
\hline & $\begin{array}{l}T_{\mathrm{mb}}{ }^{\mathrm{c}} \\
(\mathrm{K})\end{array}$ & $\begin{array}{c}\Delta v_{\text {int }} \mathrm{d}^{\mathrm{d}} \\
\left(\mathrm{km} \mathrm{s}^{-1}\right)\end{array}$ & $\tau^{\mathrm{e}}$ & $\begin{array}{c}\left\langle\Delta v_{\text {int }}\right\rangle^{\mathrm{d}} \\
\left(\mathrm{km} \mathrm{s}^{-1}\right)\end{array}$ & $\langle\tau\rangle^{\mathrm{e}}$ & $\begin{array}{c}\left\langle T_{\mathrm{ex}}\right\rangle \\
(\mathrm{K})\end{array}$ \\
\hline $\mathrm{N}_{2} \mathrm{H}^{+}(1-0)$ & $1.96 \pm 0.05$ & $0.35 \pm 0.01$ & $3.4 \pm 0.08$ & $0.32 \pm 0.09$ & $2.7 \pm 0.4$ & $9.5 \pm 1.9$ \\
\hline $\mathrm{H}^{13} \mathrm{CO}^{+}(1-0)$ & $0.88 \pm 0.09$ & $0.60 \pm 0.03$ & $\leq 0.1$ & $0.60 \pm 0.17$ & $\ldots$ & $\ldots$ \\
\hline $\mathrm{C}_{3} \mathrm{H}_{2} 2_{12}-1_{01} \ldots \ldots \ldots \ldots \ldots \ldots \ldots \ldots \ldots \ldots \ldots \ldots$ & $1.12 \pm 0.06$ & $0.59 \pm 0.1$ & 0.34 & $\ldots$ & $\cdots$ & $\ldots$ \\
\hline $\mathrm{CCS} 4_{3}-3_{2} \ldots \ldots \ldots \ldots \ldots \ldots \ldots \ldots \ldots \ldots \ldots \ldots \ldots \ldots$ & $0.74 \pm 0.07$ & $0.41 \pm 0.04$ & 0.94 & $0.41 \pm 0.19$ & $\ldots$ & $\ldots$ \\
\hline $\mathrm{NH}_{3}(1,1) \ldots \ldots \ldots \ldots \ldots$ & $1.20 \pm 0.06$ & $0.61 \pm 0.10$ & $1.6 \pm 0.1$ & $0.62 \pm 0.17$ & $0.45 \pm 0.18$ & $\leq 9.7$ \\
\hline
\end{tabular}

${ }^{a}$ Single pointing toward the $3 \mathrm{~mm}$ continuum source. See the spectra in Fig. 2 and beam sizes in Table 1 .

${ }^{\mathrm{b}}$ For $\mathrm{N}_{2} \mathrm{H}^{+}$and $\mathrm{NH}_{3}$ lines that allow us to derive optical depth $(\tau)$ and excitation temperature $\left(T_{\text {ex }}\right)$ through HFS analysis, we present mean and standard deviation for the spectra existing inside the half-maximum, i.e., $50 \%$ level contour of total integrated intensity map (Fig. 4).

${ }^{c}$ Peak main-beam brightness temperature. For $\mathrm{N}_{2} \mathrm{H}^{+}$and $\mathrm{NH}_{3}$ lines, those for the brightest $\mathrm{HF}$ components are given.

${ }^{d}$ In calculating intrinsic velocity width $\left(\Delta v_{\text {int }}\right)$, we employed HFS analysis for the $\mathrm{N}_{2} \mathrm{H}^{+}$and $\mathrm{NH}_{3}$ lines, spectral moment analysis for the $\mathrm{H}^{13} \mathrm{CO}^{+}$and $\mathrm{C}_{3} \mathrm{H}_{2}$ lines, and single Gaussian fitting for the CCS line $(\S 4.5 .2)$. For the $\mathrm{C}_{3} \mathrm{H}_{2}$ and $\mathrm{CCS}$ lines, we corrected for line broadening due to the optical depth (Phillips et al. 1979).

${ }^{\mathrm{e}}$ Optical depth. We present $\tau$ of the brightest $\mathrm{HF}$ transition $\left(F_{1}, F\right)=(2,3)-(1,2)$ for $\mathrm{N}_{2} \mathrm{H}^{+}(1-0)(\S 4.1 .1)$ and $\left(F, F_{1}\right)=(5 / 2,2)-(5 / 2,2)$ for $\mathrm{NH}_{3}(1,1)(\S 4.1 .5)$.

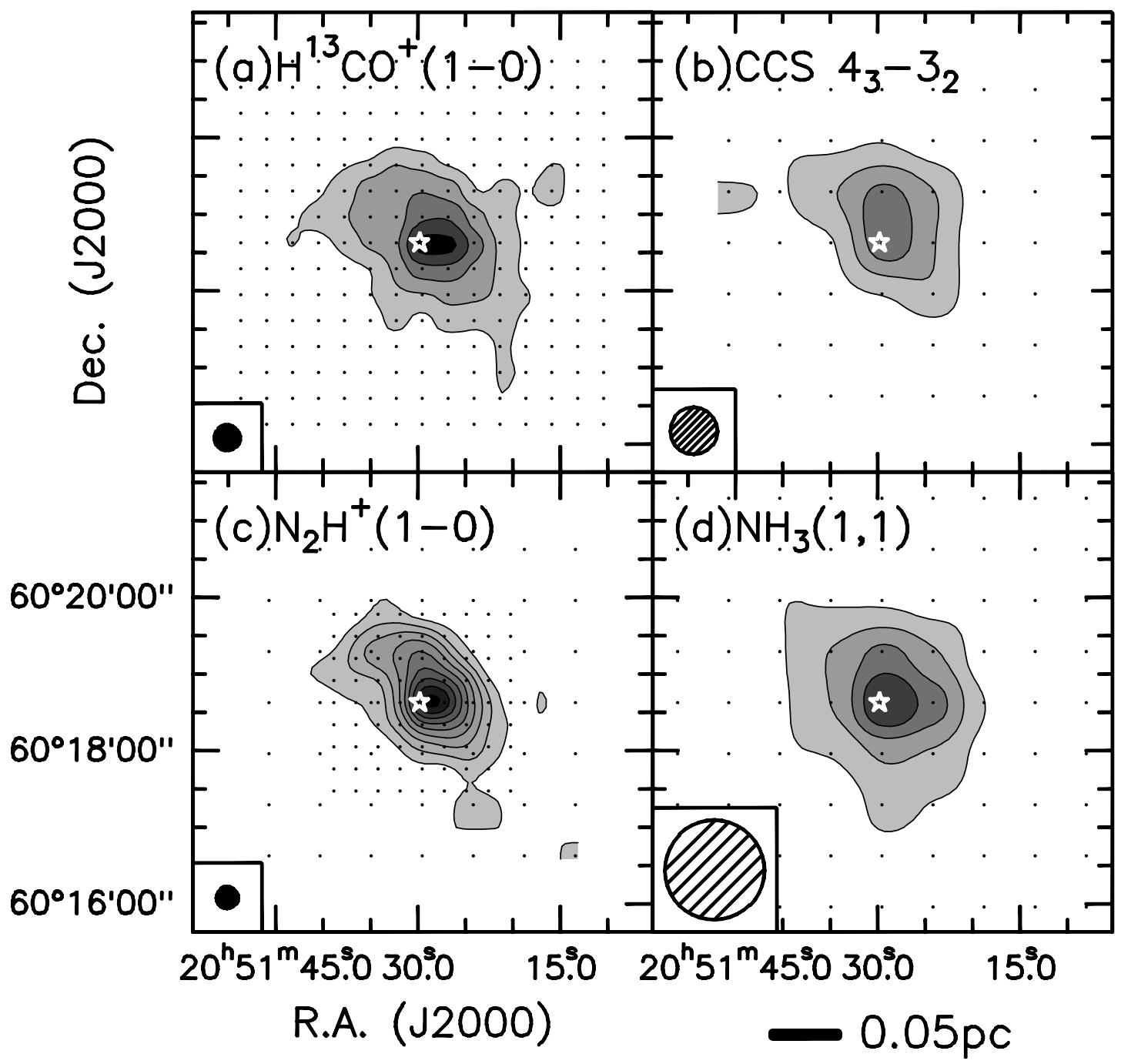

FIG. 4.-Total integrated intensity maps of the $(a) \mathrm{H}^{13} \mathrm{CO}^{+}(1-0),(b) \mathrm{CCS} 4_{3}-3_{2},(c) \mathrm{N}_{2} \mathrm{H}^{+}(1-0)$, and $(d) \mathrm{NH}_{3}(1,1)$ lines observed with the $45 \mathrm{~m}$ telescope. The velocity ranges for the integrations are between $V_{\mathrm{LSR}}=-2.95$ and $-2.1 \mathrm{~km} \mathrm{~s}^{-1}$ for $\mathrm{H}^{13} \mathrm{CO}^{+}$and between -3.0 and $-2.1 \mathrm{~km} \mathrm{~s}^{-1}$ for CCS. For the $\mathrm{N}_{2} \mathrm{H}^{+}$and $\mathrm{NH}_{3}$ emission, all of the $\mathrm{HF}$ emission is integrated over the velocity ranges shown by the horizontal bars in Figs. 3 and 10, respectively. Contour intervals are $3 \sigma$, starting from the $3 \sigma$ levels. The $1 \sigma$ noise levels are $33.4,42.5,27.0$, and $24.8 \mathrm{mK} \mathrm{km} \mathrm{s}^{-1}$ for the $\mathrm{H}^{13} \mathrm{CO}^{+}, \mathrm{CCS}, \mathrm{N}_{2} \mathrm{H}^{+}$, and $\mathrm{NH}_{3}$ maps, respectively. The central star marks the position of the $3 \mathrm{~mm}$ source. The hatched circles at the bottom left corner of each panel show beam size (see Table 1), and the small dots represent the observed grid points. 

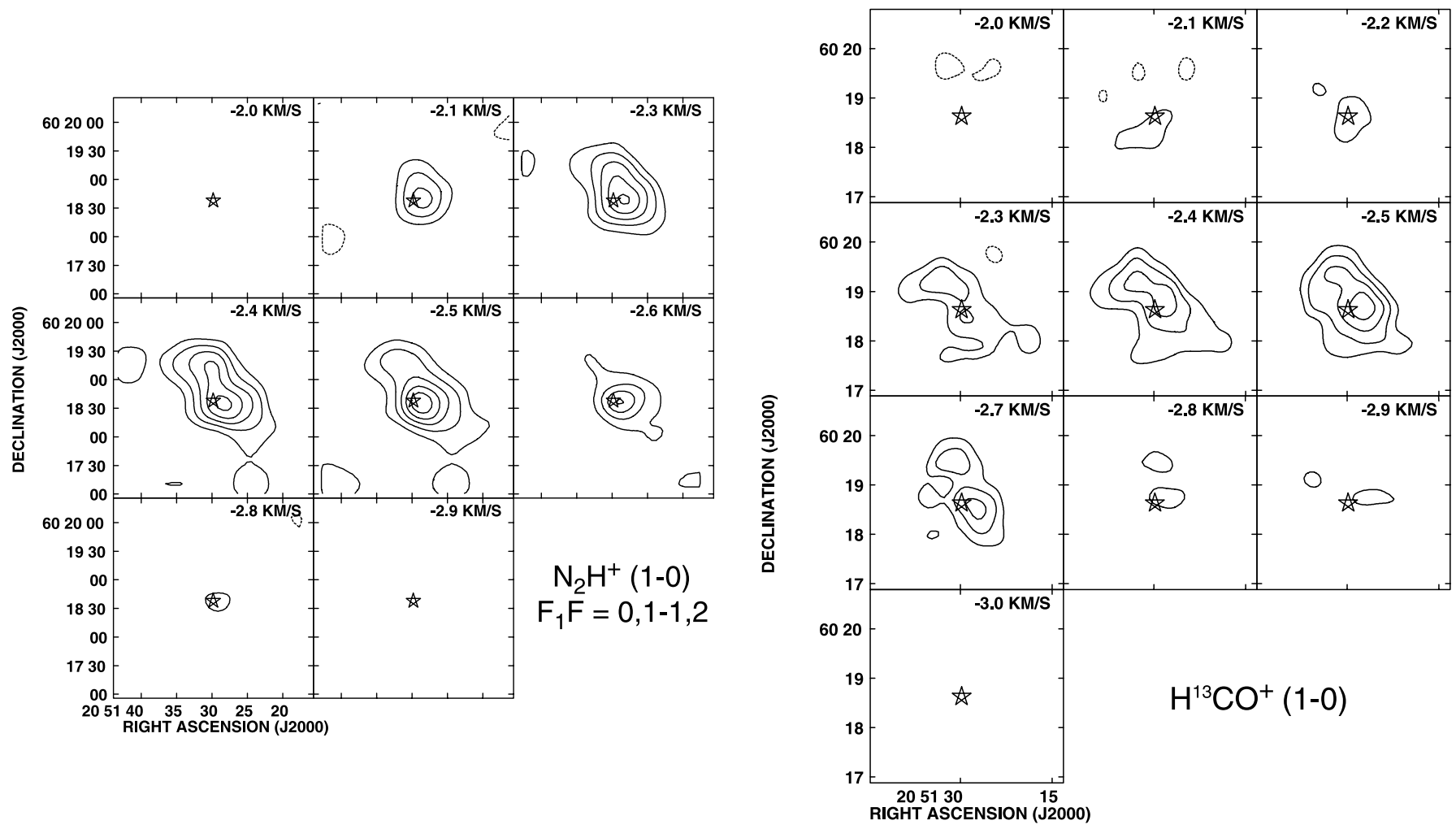

FIG. 5.- Velocity channel maps of the $\mathrm{N}_{2} \mathrm{H}^{+}(1-0)\left(F_{1}, F\right)=(0,1)-(1,2)$ and $\mathrm{H}^{13} \mathrm{CO}^{+}(1-0)$ emission taken with the $45 \mathrm{~m}$ telescope (see Table 1); each panel has a size of $225^{\prime \prime} \times 225^{\prime \prime}$. The central LSR velocity for each channel is shown in the upper right corner. Contour intervals are $2 \sigma$, starting from the $3 \sigma$ levels of the images where $1 \sigma$ noise level is $78 \mathrm{mK}$ in $T_{\mathrm{mb}}$ for the $\mathrm{N}_{2} \mathrm{H}^{+}$and $96 \mathrm{mK}$ for $\mathrm{H}^{13} \mathrm{CO}^{+}$. The star in each panel is the same as in Fig. 4. The systemic velocity is $V_{\mathrm{LSR}}=-2.5 \mathrm{~km} \mathrm{~s} \mathrm{~s}^{-1}$.

they have stronger blue peaks, a typical signature of infall (e.g., Walker et al. 1986). The $\mathrm{C}_{3} \mathrm{H}_{2}$ spectrum shows a flat-top shape. Lastly, we point out that no molecular line emission is seen around $V_{\text {LSR }}=5.7 \mathrm{~km} \mathrm{~s}^{-1}$ where we detected the weak $\mathrm{H}_{2} \mathrm{O}$ masers (Furuya et al. 2003). This may suggest that the masers are excited in a shocked region impacted by a possible protostellar jet because such jet-origin water masers in low-mass YSOs usually do not appear around their cloud velocities ( Terebey et al. 1992; Chernin 1995; Claussen et al. 1998; Moscadelli et al. 2006).

\subsubsection{Single-Dish Maps of High-Density Gas}

Figure 4 illustrates total integrated intensity maps of the highdensity gas tracers of $\mathrm{H}^{13} \mathrm{CO}^{+}(1-0), \mathrm{CCS} 4_{3}-3_{2}, \mathrm{~N}_{2} \mathrm{H}^{+}(1-0)$, and $\mathrm{NH}_{3}$ lines taken with the $45 \mathrm{~m}$ telescope. We integrated all of the HF components for the nitrogen-bearing molecules having hyperfine structures (HFSs). The four lines show similar overall structures. It is very likely that all of the molecular lines trace a natal cloud core of GF 9-2. The highest contour of the $\mathrm{H}^{13} \mathrm{CO}^{+}$ and $\mathrm{N}_{2} \mathrm{H}^{+}$emission shows east-west elongation. In addition, the $\mathrm{N}_{2} \mathrm{H}^{+}$map shows a higher degree of central concentration than the $\mathrm{H}^{13} \mathrm{CO}^{+}$and $\mathrm{CCS}$ maps with comparable beam sizes. The $3 \mathrm{~mm}$ dust continuum emission is located at the densest innermost region of the core, although its position is slightly displaced to the east of the $\mathrm{H}^{13} \mathrm{CO}^{+}$and $\mathrm{N}_{2} \mathrm{H}^{+}$peaks.

Figure 5 shows velocity channel maps of the $\mathrm{H}^{13} \mathrm{CO}^{+}(1-0)$ and $\mathrm{N}_{2} \mathrm{H}^{+}(1-0)\left(F_{1}, F\right)=(0,1)-(1,2)$ lines observed with the $45 \mathrm{~m}$ telescope; the latter transition is not blended with the other six HF components. The $\mathrm{H}^{13} \mathrm{CO}^{+}$channel maps exhibit a velocity gradient along the major axis of the core; the redshifted gas dominantly lies to the northeast of the $3 \mathrm{~mm}$ source, whereas the blueshifted one lies to the southwest.

\subsubsection{Combined OVRO Millimeter Array and Nobeyama $45 \mathrm{~m}$ Telescope Data}

Figure 6 shows the combined $\mathrm{N}_{2} \mathrm{H}^{+}$and $\mathrm{H}^{13} \mathrm{CO}^{+}$images together with the original $45 \mathrm{~m}$ and OVRO maps, and Figure 7 shows channel maps of the combined data. We note that the OVRO and combined images are insensitive to the emission outside the fields of view of the OVRO array due to primary beam attenuation of the element antennas and that the combined $\mathrm{H}^{13} \mathrm{CO}^{+}$ and $\mathrm{N}_{2} \mathrm{H}^{+}$images are $\simeq 4$ and 15 times less sensitive compared to the $45 \mathrm{~m}$ maps, respectively. The OVRO images in Figure 6 trace the innermost region of the core and clearly show that both the $\mathrm{N}_{2} \mathrm{H}^{+}$and $\mathrm{H}^{13} \mathrm{CO}^{+}$peaks are shifted to the west of the $3 \mathrm{~mm}$ source. The combined images in Figure 6 show that there is a centrally peaked feature that is elongated east-west in the smaller scale and that intensity of the two lines decreases with increasing radial distance from the center. However, contrary to Figure 5, one cannot see a well-defined velocity gradient in the combined channel maps of the $\mathrm{H}^{13} \mathrm{CO}^{+}$emission (Fig. 7).

\subsection{Search for Molecular Outflows and Jets}

\subsubsection{Search for CO Outflow}

Figure 8 compares the blue- and redshifted ${ }^{12} \mathrm{CO}(1-0)$ and (3-2) emission integrated over the velocity ranges shown in Figure 2 . In the figure, no highly collimated high-velocity "jetlike" outflow typically seen in Class 0 sources (Bachiller 1996 and references therein) is identified toward the $3 \mathrm{~mm}$ source, although formation of a protostar in the GF 9-2 core is strongly suggested by the $\mathrm{H}_{2} \mathrm{O}$ masers. This is because the terminal velocity of the blueand redshifted emission, shifted by only $\simeq 3 \mathrm{~km} \mathrm{~s}^{-1}$ to $V_{\text {sys }}$ (Fig. 2), is too small to be interpreted as the line-of-sight components of 


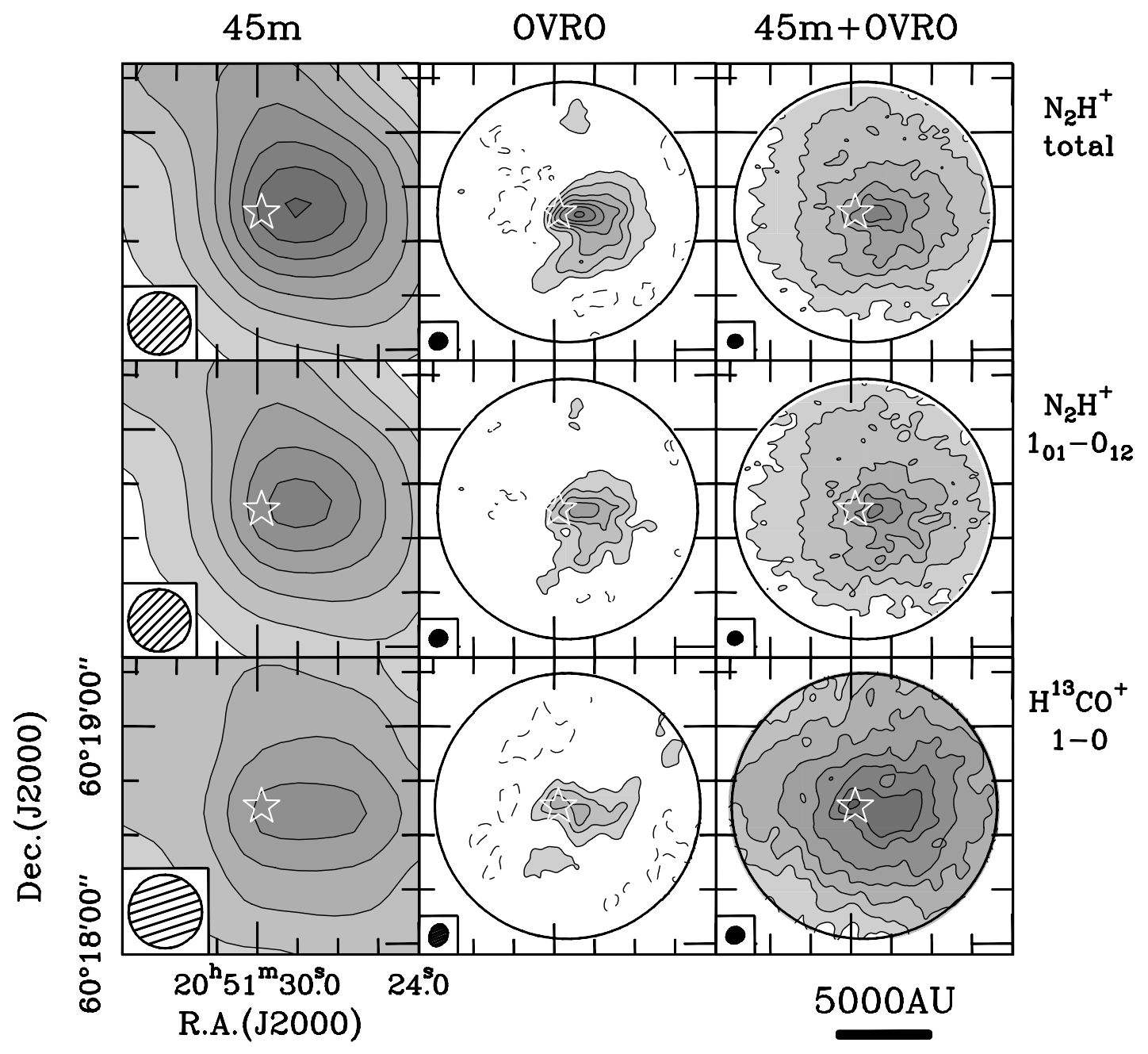

Fig. 6. - Comparison of the total integrated intensity maps of all seven $\mathrm{HF}$ emission components of $\mathrm{N}_{2} \mathrm{H}^{+}(1-0)$ (top row), $\mathrm{N}_{2} \mathrm{H}^{+}(1-0)\left(F_{1}, F\right)=(0,1)-(1,2)$ (middle row), and $\mathrm{H}^{13} \mathrm{CO}^{+}(1-0)$ (bottom row) taken with the Nobeyama $45 \mathrm{~m}$ telescope (left column), the OVRO millimeter array (middle column), and made from the combined $45 \mathrm{~m}$ plus OVRO visibilities (right column). The central star and the ellipse at the bottom left corner in each panel are the same as in Fig. 4. The large circles in the middle and right columns indicate the FOV. The $\mathrm{N}_{2} \mathrm{H}^{+}$total emission maps are integrated over the three velocity ranges shown by the horizontal bars in Fig. 3, and those for $\mathrm{N}_{2} \mathrm{H}^{+}$ $\left(F_{1}, F\right)=(0,1)-(1,2)$ and $\mathrm{H}^{13} \mathrm{CO}^{+}$maps by the green ones in Fig. 2. The contour intervals are the same as in Fig. 1 . The $1 \sigma \mathrm{rms}$ noise levels are $38.5 \mathrm{mK}$ in $T_{\mathrm{mb}}$ for the $45 \mathrm{~m}$ $\mathrm{N}_{2} \mathrm{H}^{+}$total emission map, $42.5 \mathrm{mK}$ for the $45 \mathrm{~m} \mathrm{~N}_{2} \mathrm{H}^{+}\left(F_{1}, F\right)=(0,1)-(1,2)$ map, $33.5 \mathrm{mK}$ for the $45 \mathrm{~m} \mathrm{H}^{13} \mathrm{CO}^{+}$map, $17.4 \mathrm{mJy}$ beam ${ }^{-1}$ for the OVRO N $\mathrm{O}^{+}$total emission image, $22.4 \mathrm{~m} \mathrm{Jy} \mathrm{beam}^{-1}$ for the OVRO $\mathrm{N}_{2} \mathrm{H}^{+}\left(F_{1}, F\right)=(0,1)-(1,2)$ image, $17.2 \mathrm{mJy}_{\text {beam }}{ }^{-1}$ for the OVRO $\mathrm{H}^{13} \mathrm{CO}^{+}$image, $62.4 \mathrm{mJy}$ beam ${ }^{-1}$ for the combined $\mathrm{N}_{2} \mathrm{H}^{+}$ total emission image, $95.5 \mathrm{mJy}_{\text {beam }}^{-1}$ for the combined $\mathrm{N}_{2} \mathrm{H}^{+}\left(F_{1}, F\right)=(0,1)-(1,2)$ image, and $35.3 \mathrm{mJy}_{\text {beam }}^{-1}$ for the combined $\mathrm{H}^{13} \mathrm{CO}^{+}$image. The noise levels for the combined data correspond to $170 \mathrm{mK}$ in $T_{\mathrm{sb}}$ for the $\mathrm{H}^{13} \mathrm{CO}^{+}, 510 \mathrm{mK}$ for the $\mathrm{N}_{2} \mathrm{H}^{+}$total, and $780 \mathrm{mK}$ for the $\mathrm{N}_{2} \mathrm{H}^{+}\left(F_{1}, F\right)=(0,1)-(1,2)$ emission maps.

$\mathrm{CO}$ outflow velocity $\left(V_{\text {flow }}\right)$ in Class 0 sources (e.g., $V_{\text {flow }} \simeq$ $50 \mathrm{~km} \mathrm{~s}^{-1}$ for VLA 1623; André et al. 1990). Instead, a condensation of the low-velocity redshifted gas lies to the south of the $3 \mathrm{~mm}$ source in the (3-2) map. A similar condensation is marginally seen in the redshifted (1-0) map but seems to be a part of the large-scale natal filament. These red condensations might represent a compact low-velocity outflow that has just launched from a protostar. On the other hand, a patchy distribution of the blueshifted (3-2) gas is observed, partly because of the quality of spectral baselines. The blueshifted (1-0) map shows a ridge to the northeast of the $3 \mathrm{~mm}$ source and is connected to the east-west natal filament, whereas the blueshifted (3-2) emission seems to be resolved into two peaks in the core center.

First, we estimate an upper limit to the mass of the highvelocity outflow gas. Since the $\mathrm{CO}$ emission is very likely to be optically thick, we consider that the peak brightness temperature gives a good estimate of $T_{\mathrm{ex}}$, assuming that the emission fills the beam. We derive $T_{\text {ex }}$ of 5.9 and $6.9 \mathrm{~K}$ from the (1-0) and (3-2) transitions, respectively, considering the cosmic background tem- perature $\left(T_{\mathrm{bg}}=2.7 \mathrm{~K}\right)$. We attained $3 \sigma$ upper limits of 12 and $79 \mathrm{mK}$ for the (1-0) and (3-2) transitions, respectively, with a velocity width of $50 \mathrm{~km} \mathrm{~s}^{-1}$, which is a typical velocity of highvelocity jetlike outflows associated with Class 0 sources (e.g., Bachiller 1996), although Figure 2 shows that terminal velocity is $\simeq 3 \mathrm{~km} \mathrm{~s}^{-1}$. Given the mean value of $T_{\text {ex }}=6.4 \mathrm{~K}$ and $[\mathrm{CO}] /\left[\mathrm{H}_{2}\right] \simeq 10^{4}$ (Dickman 1978), we convert these upper limits into mass per lobe $\left(M_{\text {lobe }}\right)$ of $6.7 \times 10^{-5} M_{\odot}$ for $(1-0)$ and $1.7 \times$ $10^{-3} M_{\odot}$ for (3-2). Clearly, these $3 \sigma$ detection limits in mass are sufficient to identify any of the $\mathrm{CO}$ outflows associated with Class 0 sources known to date (e.g., Richer et al. 2000).

Next, we estimate mass of the possible low-velocity $\left(V_{\text {flow }} \simeq\right.$ $3 \mathrm{~km} \mathrm{~s}^{-1}$ ) outflow suggested by the weak wing emission in Figure 2 and by the redshifted condensation in Figure 8. The extents of the lobes are approximately 0.053 and $0.032 \mathrm{pc}$ for the blue and red ones from the $3 \mathrm{~mm}$ source, respectively. Given $T_{\mathrm{ex}}=6.4 \mathrm{~K}$, we obtain $M_{\text {lobe }}$ of $1.6 \times 10^{-4} M_{\odot}$ for the blue and $2.6 \times 10^{-4} M_{\odot}$ for the red. These lobe masses are considerably smaller than those in Class 0 sources. 

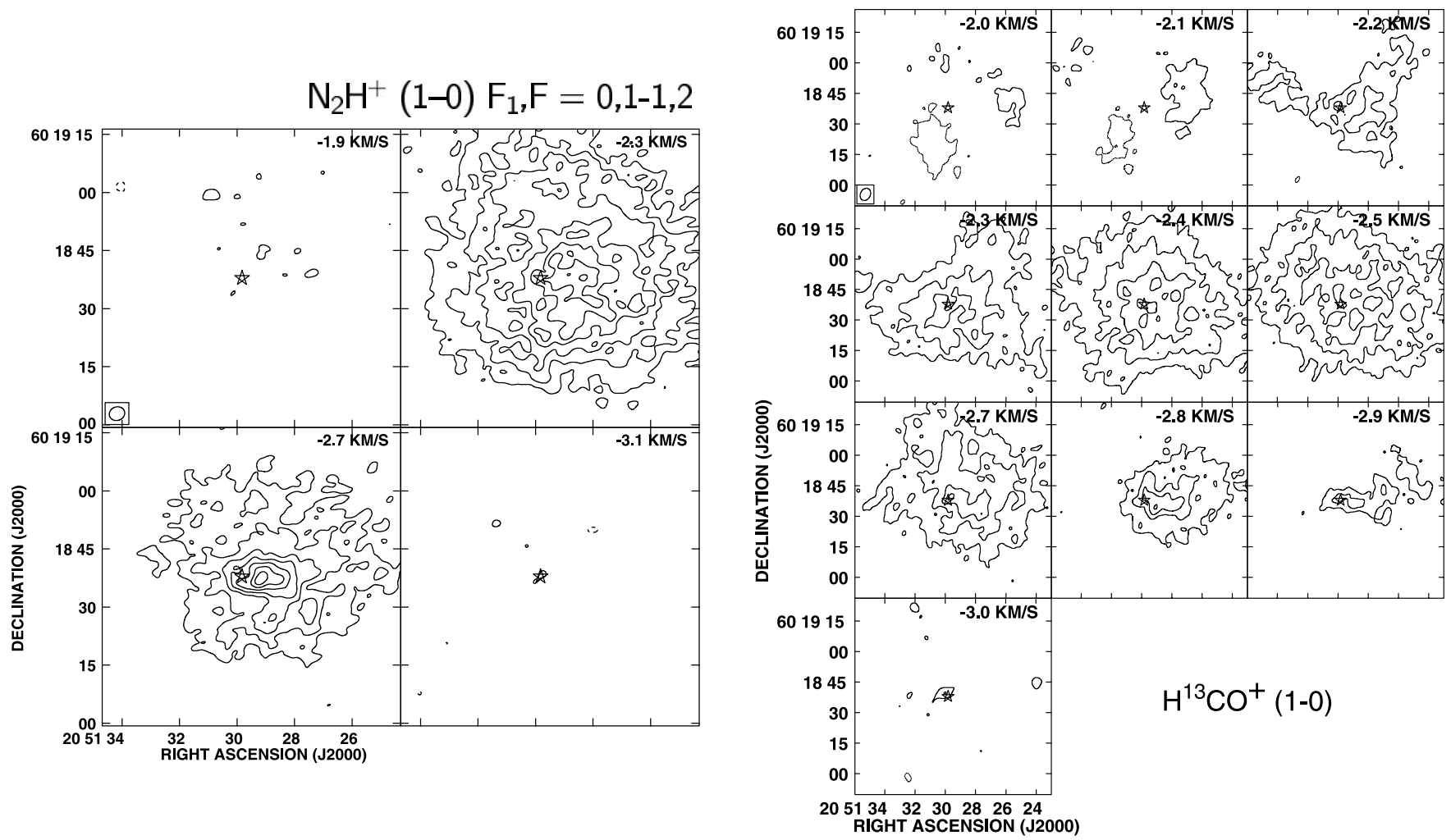

FIG. 7.- Velocity channel maps of the combined OVRO plus $45 \mathrm{~m}$ telescope images of the $\mathrm{N}_{2} \mathrm{H}^{+}(1-0)\left(F_{1}, F\right)=(0,1)-(1,2)$ and $\mathrm{H}^{13} \mathrm{CO}^{+}(1-0)$ lines $($ see $\mathrm{Table} 3)$. Contour intervals are the same as in Fig. 1, and the rms noise levels are 32.1 and $52.1 \mathrm{mJy}$ beam ${ }^{-1}$ for the $\mathrm{N}_{2} \mathrm{H}^{+}$and $\mathrm{H}^{13} \mathrm{CO}^{+}$lines, respectively. The size for each channel map is $76 . " 8 \times 76$ " 8 . The systemic velocity is $V_{\mathrm{LSR}}=-2.5 \mathrm{~km} \mathrm{~s}^{-1}$.

\subsubsection{Nondetection of Shock-excited $\mathrm{SiO}$ Emission}

Thermal $\mathrm{SiO}$ emission is believed to be one of the most reliable shock tracers associated with molecular outflow (e.g., Codella et al. 1999) because the destruction of dust grains releases silicon into the gas phase and silicon is rapidly oxidized to produce $\mathrm{SiO}$ (e.g., Schilke et al. 1997). No evidence of outflow shocked regions is found in the $\mathrm{SiO}$ emission over a $0.22 \mathrm{pc} \times 0.14 \mathrm{pc}$ region centered on the $3 \mathrm{~mm}$ continuum source. The searched area is sufficiently large to detect such $\mathrm{SiO}$ emission enhanced at the leading edge of the possible outflow lobe younger than the dynamical age of $\lesssim 0.053 \mathrm{pc} / 3 \mathrm{~km} \mathrm{~s}^{-1} \simeq 1.7 \times 10^{4} \mathrm{yr}$. This result suggests that the compact low-velocity outflow $(\S 3.3 .1)$ is not powerful enough to excite thermal $\mathrm{SiO}$ emission.

\section{ANALYSIS}

\subsection{Column Density of the Core}

\subsection{1. $\mathrm{N}_{2} \mathrm{H}^{+}(1-0)$}

To estimate the column density of $\mathrm{N}_{2} \mathrm{H}^{+}\left(N_{\mathrm{N}_{2} \mathrm{H}^{+}}\right)$, we analyze the HFS due to the electric quadrupole moment of the nitrogen nucleus. We performed least-squares fitting to each spectrum by applying a model spectrum having seven Gaussian components assumed to have the intrinsic intensity ratios in local thermodynamic equilibrium (LTE) at the limit of $\tau=0$ (Cazzoli et al. 1985). The details of the HFS analysis and the column density calculations are described in $\S \mathrm{B} 1$. The free parameters are $T_{\mathrm{ex}}$, velocity width $\left(\Delta v_{\mathrm{FWHM}}\right)$, LSR velocity of the brightest HF component $\left[J=1-0\left(F_{1}, F\right)=(2,3)-(1,2)\right.$ transition at $\left.93173.809 \mathrm{MHz}\right]$, and the sum of the optical depths of the components $\left(\tau_{\text {tot }}\right)$. We have presented an example $\mathrm{N}_{2} \mathrm{H}^{+}$spectrum toward the $3 \mathrm{~mm}$ peak position in Figure 3. We used only the single-dish data because the velocity resolution is 3.4 times higher than that of the combined data. The parameters of the best-fit model profiles for the HF spectrum, shown by the green histogram, are summarized in Table 5.

To perform HFS analysis on the combined data, we create a spectrum at each map pixel from the three-dimensional cube data. The pixel size is set to be 0.9 , the maximum size to avoid aliasing in the fast Fourier transform (FFT) of the combined visibilities, whereas that for the $45 \mathrm{~m}$ data is set to be $5^{\prime \prime}$. We obtained reasonable fitting results from 262 and 1776 spectra for the $45 \mathrm{~m}$ and combined data, respectively. For the remaining spectra, we failed to achieve a fit because the weakest HF component of $\left(F_{1}, F\right)=$ $(0,1)-(1,2)$ whose intrinsic intensity is 0.111 times that of the brightest $\left(F_{1}, F\right)=(2,3)-(1,2)$ did not have $\mathrm{S} / \mathrm{N}$ higher than 5 .

Figure 9 shows total column density maps of $\mathrm{N}_{2} \mathrm{H}^{+}$calculated from the $45 \mathrm{~m}$ and combined data. To produce Figure 9, we calculated $N_{\mathrm{N}_{2} \mathrm{H}^{+}}$at each pixel using equation (B7), where we used $T_{\text {ex }}, \tau_{\text {tot }}$, and intrinsic velocity width, $\Delta v_{\text {int }}$, derived from the HFS analysis of the $\mathrm{N}_{2} \mathrm{H}^{+}$spectrum at a pixel. The $45 \mathrm{~m} \mathrm{~N}_{\mathrm{N}_{2} \mathrm{H}^{+}}$map shows that the core has the densest region at the center and that the column density decreases outward. The combined $\mathrm{N}_{2} \mathrm{H}^{+}$map shows that the extent of the densest central region is smaller than the $45 \mathrm{~m}$ beam size. The region has an approximate extent of $10^{\prime \prime}$ (corresponding to $0.01 \mathrm{pc}$ ) and $N_{\mathrm{N}_{2} \mathrm{H}^{+}}$of $\gtrsim 10^{14} \mathrm{~cm}^{-2}$. Notice that the $3 \mathrm{~mm}$ source is shifted by $6^{\prime \prime}$ to the east of the $N_{\mathrm{N}_{2} \mathrm{H}^{+}}$ peak.

To obtain fractional abundances of the probe molecules in $\S 4.2$, we recalculate $N_{\mathrm{N}_{2} \mathrm{H}^{+}}$with various beam sizes. We smoothed the $45 \mathrm{~m}$ data by $40^{\prime \prime}$ and $80^{\prime \prime}$ beams for $r \leq 20^{\prime \prime}$ and $40^{\prime \prime}$ regions centered on the $3 \mathrm{~mm}$ source, respectively. Table 6 summarizes the calculated $N_{\mathrm{N}_{2} \mathrm{H}^{+}}$and compares CCS and $\mathrm{NH}_{3}$ column densities. We also reconstructed $\mathrm{N}_{2} \mathrm{H}^{+}$images from the combined 

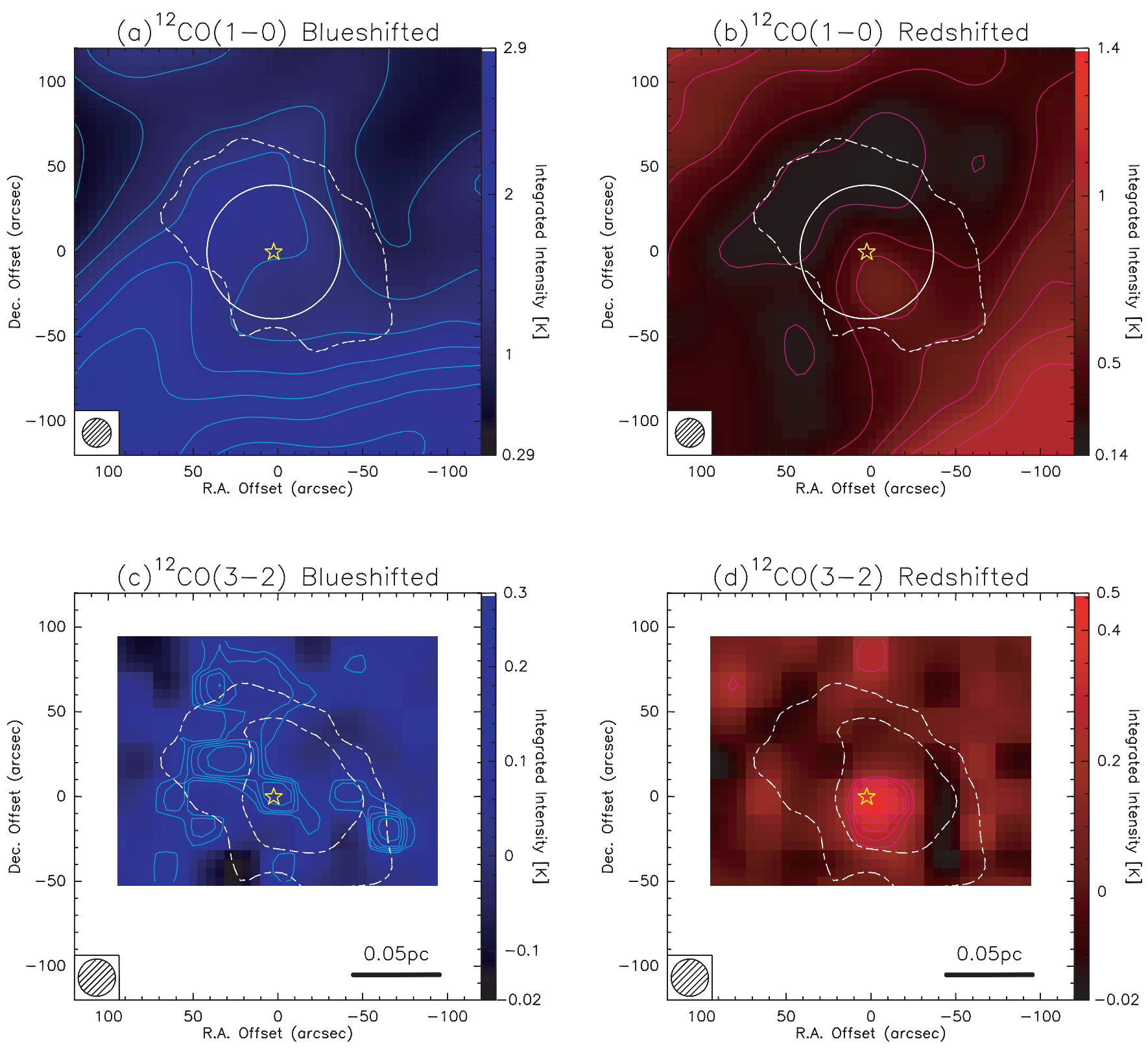

Fig. 8. - Maps of the blueshifted (left) and redshifted (right) ${ }^{12} \mathrm{CO}(1-0)($ top $)$ and $(3-2)($ bottom $)$ emission. The (1-0) emission is integrated over the velocity ranges of $-4.2 \leq V_{\mathrm{LSR}} / \mathrm{km} \mathrm{s}^{-1} \leq-3.4$ for the blue and $-1.8 \leq V_{\mathrm{LSR}} / \mathrm{km} \mathrm{s}^{-1} \leq-1.0$ for the red. The (3-2) emission is integrated in the ranges of $-5.8 \leq V_{\mathrm{LSR}} / \mathrm{km} \mathrm{s}^{-1} \leq-3.8$ for the blue and $-1.4 \leq V_{\mathrm{LSR}} / \mathrm{km} \mathrm{s}^{-1} \leq+0.6$ for the red. These velocity ranges are shown in Fig. 2. Contours with color scale are drawn by the $10 \%$ step up to the $90 \%$ level of the peak value. The lowest contour for the (1-0) maps is the $10 \%$ level, corresponding to the 21 and $8.4 \sigma$ levels for the blue and red, respectively. In the (3-2) maps, the lowest contour is the $50 \%$ level, corresponding to the 9.0 and $8.8 \sigma$ levels for the blue and red, respectively. The white dashed contours shown in all of the panels indicate the $5 \sigma$ level contour of the $\mathrm{H}^{13} \mathrm{CO}^{+}$total intensity map ( Fig. $4 a$ ), and the inner ones in $(c)$ and $(d)$ indicate the $50 \%$ (corresponding to $16.4 \sigma$ ) level contour. The central circles with a radius of $40^{\prime \prime}$ in $(a)$ and $(b)$ show the error circle of the $\mathrm{H}_{2} \mathrm{O}$ maser position (Furuya et al. 2003). The central yellow star indicates the position of the $3 \mathrm{~mm}$ source. The other symbols are the same as in Fig. 4.

visibilities by giving the SHARC II beam as a synthesized beam size and calculated $N_{\mathrm{N}_{2} \mathrm{H}^{+}}$inside the $3 \sigma$ level contour of the $350 \mu \mathrm{m}$ emission.

In addition, we analyzed the $\mathrm{N}_{2} \mathrm{H}^{+}$line data with the $45 \mathrm{~m}$ telescope to use the line as a temperature probe for the other molecules because the $\mathrm{N}_{2} \mathrm{H}^{+} \mathrm{HFS}$ analysis gives rather accurate estimates of $T_{\text {ex }}$ and the spatial resolution for $\mathrm{N}_{2} \mathrm{H}^{+}$is the highest among our data. We analyzed $\mathrm{N}_{2} \mathrm{H}^{+}$spectra inside the region enclosed by the half-maximum, i.e., $50 \%$ level contour to the peak intensity, and obtained mean $T_{\text {ex }}$ of $9.5 \pm 1.9 \mathrm{~K}$ (Table 5). We assume that the core has a temperature of $9.5 \mathrm{~K}$ and use the temperature to calculate the column density of the other molecules. Note that the adopted temperature is higher than the range of $5-7 \mathrm{~K}$ for the ambient gas inferred from the ${ }^{12} \mathrm{CO}(3-2)$ and (1-0) emission (§ 3.3.1).

\subsection{2. $\mathrm{H}^{13} \mathrm{CO}^{+}(1-0)$}

To estimate the column density of $\mathrm{H}^{13} \mathrm{CO}^{+}\left(N_{\mathrm{H}^{13} \mathrm{CO}^{+}}\right)$, we analyzed both the $45 \mathrm{~m}$ telescope and combined data. We first estimate the optical depth $\left(\tau_{\mathrm{H}^{13} \mathrm{CO}^{+}}\right)$of the $\mathrm{H}^{13} \mathrm{CO}^{+}(1-0)$ line toward the core center from an antenna temperature ratio of $T_{A}^{*}\left(\mathrm{H}^{13} \mathrm{CO}^{+}\right) /$ $T_{A}^{*}\left(\mathrm{HC}^{18} \mathrm{O}^{+}\right)$. Here we assume that the beam filling factors, $T_{\mathrm{ex}}$, and the telescope beam efficiencies are the same for the two isotope lines. In addition, we assume that the solar abundance ratio 


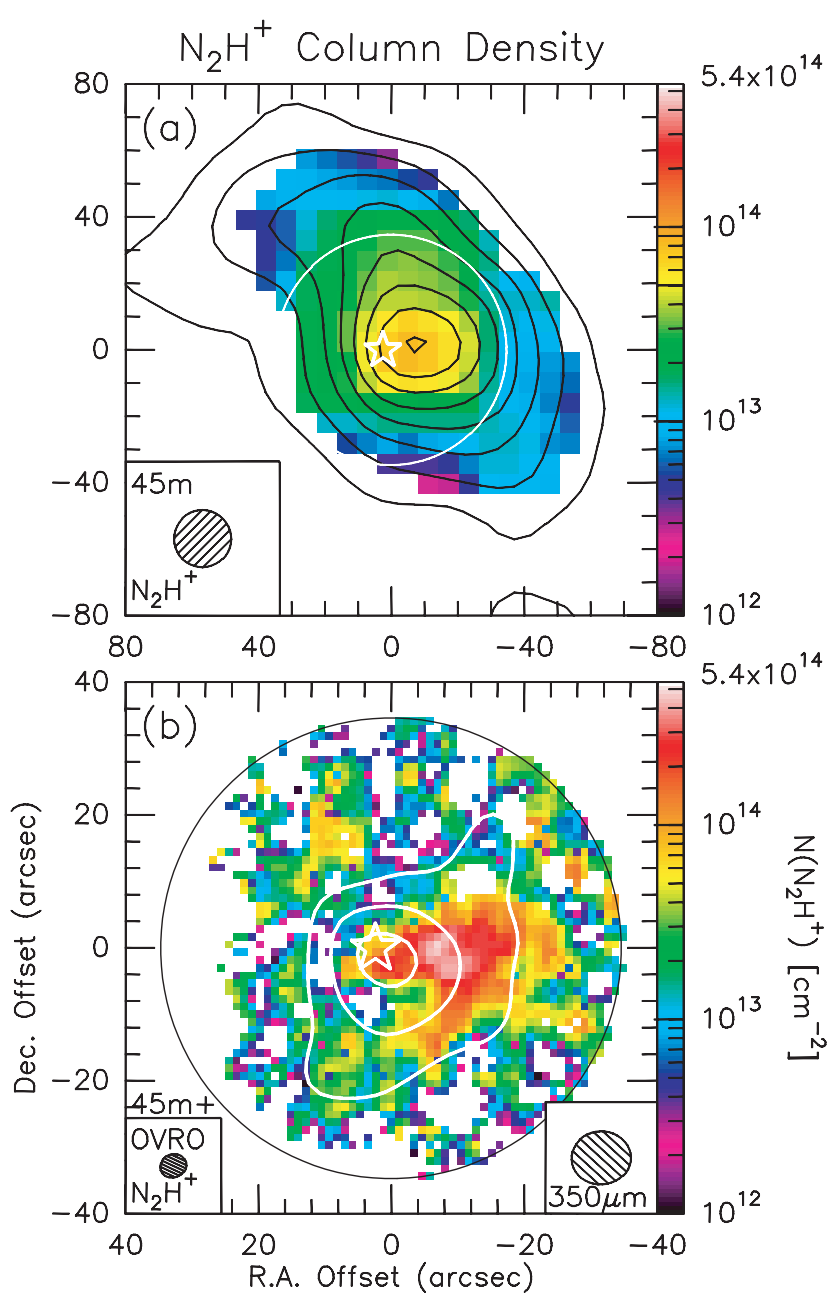

Fig. 9.-Column density maps of the $\mathrm{N}_{2} \mathrm{H}^{+}$line obtained from (a) the $45 \mathrm{~m}$ telescope data and $(b)$ the combined OVRO plus $45 \mathrm{~m}$ data $(\S 4.1)$ in color scale. The color scale bars are the same for both the $45 \mathrm{~m}$ and combined data. The black contours in $(a)$ and white ones in $(b)$ indicate the total integrated intensity maps of the $\mathrm{N}_{2} \mathrm{H}^{+}$emission (Fig. $4 c$ ) and the $350 \mu$ m continuum emission (Fig. 1), respectively. The white circle in $(a)$ and black one in $(b)$ indicate the OVRO FOV. $\left(\left[{ }^{13} \mathrm{C}\right] /\left[{ }^{12} \mathrm{C}\right]\right)\left(\left[{ }^{16} \mathrm{O}\right] /\left[{ }^{18} \mathrm{O}\right]\right)$ of 5.5 is applied to the $\left[\mathrm{H}^{13} \mathrm{CO}^{+}\right] /$ $\left[\mathrm{HC}^{18} \mathrm{O}^{+}\right]$ratio. Using the peak $T_{A}^{*}$ of $440 \mathrm{mK}$ for the $\mathrm{H}^{13} \mathrm{CO}^{+}$line in Table 5 ( $T_{\mathrm{mb}}$ is converted into $T_{A}^{*}$ by multiplying $\eta_{\mathrm{mb}}$ shown in Table 1) and the $1.5 \sigma$ upper limit of $39 \mathrm{mK}$ in $T_{A}^{*}$ for the $\mathrm{HC}^{18} \mathrm{O}^{+}$ line (Table 1), we found that the $\mathrm{H}^{13} \mathrm{CO}^{+}$line is optically thin $\left(<0.1\right.$; Table 5). Given $\tau_{\mathrm{H}^{13} \mathrm{CO}^{+}}$of $<0.1$ and $T_{\text {ex }}$ of $9.5 \mathrm{~K}(\S 4.1 .1)$, we obtained $N_{\mathrm{H}^{13} \mathrm{CO}^{+}}$from the $45 \mathrm{~m}$ and combined data (Table 6) by using equations (B9) and (B10) (see $\S \mathrm{B} 2$ ), where the SHARC II beam was used as a synthesized beam to calculate $N_{\mathrm{H}^{13} \mathrm{CO}^{+}}$inside the $3 \sigma$ contour of the $350 \mu \mathrm{m}$ emission.

\subsection{3. $\mathrm{C}_{3} \mathrm{H}_{2} 22_{12}-1_{01}$}

For calculating $\mathrm{C}_{3} \mathrm{H}_{2}$ column density $\left(N_{\mathrm{C}_{3} \mathrm{H}_{2}}\right)$ traced by the $2_{12}-1_{01}$ transition, we followed the calculations by Benson et al. (1998, see their $\S \mathrm{A} 2$ ). We observed $\mathrm{C}_{3} \mathrm{H}_{2}$ only toward the core center. We estimated the optical depth using equation (17) of Benson et al. (1998) of $\tau_{\mathrm{C}_{3} \mathrm{H}_{2}}=\ln \left[\left(T_{\mathrm{ex}}-T_{\mathrm{mb}}\right) /\left(T_{\mathrm{ex}}-T_{\mathrm{mb}}-\right.\right.$ $\left.\left.T_{\mathrm{bg}}\right)\right] ; \tau_{\mathrm{C}_{3} \mathrm{H}_{2}}=0.34$ for $T_{\mathrm{mb}}$ of $1.1 \mathrm{~K}$, assuming $T_{\mathrm{ex}}=9.5 \mathrm{~K}$ ( $\{4.1 .1)$. We derived $\Delta v_{\text {int }}$ (Table 5) from the second-order moment of the spectrum because the line profile is not a single Gaussian (see Fig. 2). Finally, a mean $\mathrm{C}_{3} \mathrm{H}_{2}$ column density is estimated to be $(2.4 \pm 0.3) \times 10^{13} \mathrm{~cm}^{-2}$ (Table 6).

\subsection{4. $\mathrm{CCS} 4_{3}-3_{2}$}

In a similar manner as the $\mathrm{H}^{13} \mathrm{CO}^{+}$analysis, we estimated the CCS column density $\left(N_{\mathrm{CCS}}\right)$ using equation (B11). We obtain $\tau_{\mathrm{CCS}}$ of 0.94 (Table 5) from a ratio of $T_{A}^{*}(\mathrm{CCS}) / T_{A}^{*}\left(\mathrm{CC}^{34} \mathrm{~S}\right)=$ 15.2, which is derived from the peak $T_{A}^{*}$ of $560 \mathrm{mK}$ for CCS (Table 5) and a $1.5 \sigma$ upper limit of $37.5 \mathrm{mK}$ for $\mathrm{CC}^{34} \mathrm{~S}$ (Table 1). Here we adopt the solar abundance ratio $\left[{ }^{32} \mathrm{~S}\right] /\left[{ }^{34} \mathrm{~S}\right]$ of 23 . Given $\tau_{\mathrm{CCS}}$ of 0.94 and $T_{\text {ex }}$ of $9.5 \mathrm{~K}$, we obtain $N_{\mathrm{CCS}}$ of $(1.5 \pm 0.2) \times$ $10^{13} \mathrm{~cm}^{-2}$ toward the core center with a $40^{\prime \prime}$ beam (Table 6 ).

$$
\text { 4.1.5. } \mathrm{NH}_{3}(1,1) \text { and }(2,2)
$$

We present spectral line profiles of the $\mathrm{NH}_{3}(J, K)=(1,1)$ and $(2,2)$ lines in Figure 10 . The $(1,1)$ spectrum consists of five groups of the lines. The most intense emission in the $(1,1)$ transition, the central group that contains eight of the HF lines, has a peak $T_{\mathrm{mb}}$ of $640 \mathrm{mK}$, whereas no $(2,2)$ emission is seen above our $3 \sigma$ level detection threshold $\left(T_{\mathrm{mb}}=210 \mathrm{mK}\right)$. Neither the $(3,3)$ nor $(4,4)$ emission was detected.

TABLE 6

Column Densities of $\mathrm{N}_{2} \mathrm{H}^{+}, \mathrm{H}^{13} \mathrm{CO}^{+}, \mathrm{C}_{3} \mathrm{H}_{2}$, CCS, and $\mathrm{NH}_{3}$ Molecules

\begin{tabular}{|c|c|c|c|c|c|}
\hline \multirow[b]{2}{*}{ LiNe } & \multirow{2}{*}{$\begin{array}{c}\text { COMBINED } \\
\begin{array}{c}\text { INSIDE } 350 \mu \mathrm{m} \\
3 \sigma \text { ConTOUR }^{\mathrm{b}} \\
\left(\mathrm{cm}^{-2}\right)\end{array}\end{array}$} & \multicolumn{4}{|c|}{ Nobeyama 45 m Telescope } \\
\hline & & $\begin{array}{l}r \leq 10^{\prime \prime} \\
\left(\mathrm{cm}^{-2}\right)\end{array}$ & $\begin{array}{l}r \leq 20^{\prime \prime} \\
\left(\mathrm{cm}^{-2}\right)\end{array}$ & $\begin{array}{l}r \leq 40^{\prime \prime} \\
\left(\mathrm{cm}^{-2}\right)\end{array}$ & $\begin{array}{l}\text { Full Extent of THE } \text { CoRE }^{\mathrm{c}} \\
\left(\mathrm{cm}^{-2}\right)\end{array}$ \\
\hline $\mathrm{N}_{2} \mathrm{H}^{+}$ & $(5.2 \pm 2.5) \mathrm{E}+13$ & $(5.6 \pm 0.2) \mathrm{E}+13$ & $(4.4 \pm 0.3) \mathrm{E}+13$ & $(3.5 \pm 0.2) \mathrm{E}+13$ & $(3.9 \pm 1.0) \mathrm{E}+13$ \\
\hline $\mathrm{H}^{13} \mathrm{CO}^{+} \ldots \ldots \ldots$ & $(4.7 \pm 0.8) \mathrm{E}+11$ & $(6.1 \pm 0.3) \mathrm{E}+11$ & $\ldots$ & $\ldots$ & $(4.3 \pm 0.2) \mathrm{E}+11$ \\
\hline $\mathrm{NH}_{3}$ & $\cdots$ & $\cdots$ & $\ldots$ & $(8.2 \pm 0.2) \mathrm{E}+14$ & $(7.7 \pm 2.8) \mathrm{E}+14$ \\
\hline
\end{tabular}

Note.-Calculated with the assumption of $T_{\mathrm{ex}}=9.5 \mathrm{~K}(\S 4.1 .1)$.

${ }^{a}$ Toward the $3 \mathrm{~mm}$ continuum source measured with the beam size in Table 1, e.g., $\theta_{\mathrm{HPBW}}=20^{\prime \prime}$ beam for the region of $r \leq 10^{\prime \prime}$. Regarding the $\mathrm{N}_{2} \mathrm{H}^{+}$column densities for the regions of $r \leq 20^{\prime \prime}$ and $40^{\prime \prime}$, we calculate column densities using these spectra smoothed with $\theta_{\mathrm{HPBW}}=40^{\prime \prime}$ and $80^{\prime \prime}$ beams, respectively ( $\S 4.1 .1$ )

${ }^{\mathrm{b}}$ Column densities measured inside an area enclosed by the $3 \sigma$ level contour of the $350 \mu \mathrm{m}$ continuum emission. For this purpose, we made the $\mathrm{N}_{2} \mathrm{H}^{+}$and $\mathrm{H}^{13} \mathrm{CO}^{+}$maps by giving the SHARC II beam size as a synthesized beam size.

c Measured over the full extent of the core with beam sizes of $20^{\prime \prime}$ for $\mathrm{N}_{2} \mathrm{H}^{+}, \mathrm{H}^{13} \mathrm{CO}^{+}$, and $\mathrm{C}_{3} \mathrm{H}_{2}, 40^{\prime \prime}$ for $\mathrm{CCS}$, and $80^{\prime \prime}$ for $\mathrm{NH}_{3}$. Here the full extent of the core is defined by a region enclosed by the $50 \%$ level contour of each total intensity map. 

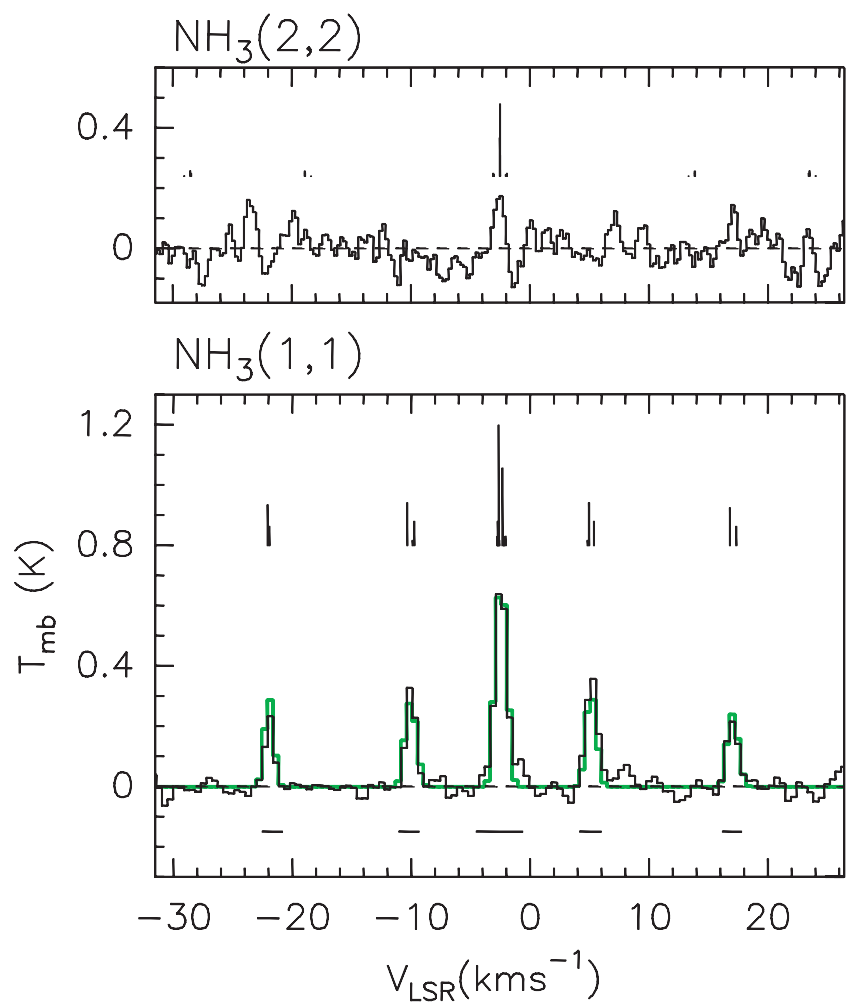

FIG. 10.- $\mathrm{NH}_{3}(1,1)$ and $(2,2)$ spectra observed with the $45 \mathrm{~m}$ telescope toward the $3 \mathrm{~mm}$ continuum emission. In the bottom panel, the green histogram represents the best-fit $\mathrm{NH}_{3}(1,1)$ spectrum from the HFS analysis $(\S 4.1 .5)$. It is assumed that the $(1,1)$ and $(2,2)$ spectra have their brightest HF components at $V_{\mathrm{LSR}}=-2.5 \mathrm{~km} \mathrm{~s}^{-1}$. The vertical lines above the spectra indicate the expected relative intensities at $\tau \simeq 0$ and LSR velocities of the HF components. The horizontal bars below the $(1,1)$ spectrum show the velocity ranges to obtain the total intensity map shown in Fig. $4 d$.

To estimate $\mathrm{NH}_{3}$ column density $\left(N_{\mathrm{NH}_{3}}\right)$, we followed the previous works, e.g., Winnewisser et al. (1979), Ungerechts et al. (1980), Stutzki et al. (1982), and Ho \& Townes (1983). We analyzed the spectra taken inside the $50 \%$ level contour of the total intensity map (Fig. $4 d$ ). In a similar manner as the $\mathrm{N}_{2} \mathrm{H}^{+}$HFS analysis, our ammonia HFS analysis gives $\tau_{\text {tot }}, \Delta v_{\mathrm{FWHM}}$, and the LSR velocity of the strongest HF component. The brightest HF emission has an optical depth of $\simeq 1.6$ toward the core center (Table 5). Here we assume that the beam filling factor is unity. It is known that rotational temperature $\left(T_{r, 21}\right)$ between the $(1,1)$ and $(2,2)$ transitions and the kinetic temperature $\left(T_{k}\right)$ agree well with each other for high-density $\left(\gtrsim 10^{6} \mathrm{~cm}^{-3}\right)$ and cold $(\lesssim 10 \mathrm{~K})$ gas ( Tafalla et al. 2004). We therefore assume $T_{r, 21}=T_{k}$ and that $T_{k}$ is equal to $T_{\text {ex }}\left(\mathrm{N}_{2} \mathrm{H}^{+}\right)$of $9.5 \mathrm{~K}(\S 4.1 .1)$. Now we calculate a mean column density of $(1.6 \pm 0.6) \times 10^{14} \mathrm{~cm}^{-2}$ for the $\mathrm{NH}_{3}$ molecule in the $(1,1)$ state inside the $50 \%$ level contour of the total integrated intensity map by inverting equation (2) of Ungerechts et al. (1980). We consequently obtained total $\mathrm{NH}_{3}$ column density from the $(1,1)$ column density by equation (2) of Winnewisser et al. (1979) (Table 6).

\subsection{Fractional Abundances of the Molecules in the Core}

To calculate mass $\left(M_{\mathrm{LTE}}\right)$ of the core from the column densities $(\S 4.1)$, we need to find the fractional abundances $\left(X_{m}\right)$ of the probe molecules to $\mathrm{H}_{2}$ molecules. Such an abundance is known to vary under different physical and chemical conditions. Bergin \& Langer (1997) theoretically predicted that all molecules are depleted in some degree from gas phase for high densities of $n\left(\mathrm{H}_{2}\right) \gtrsim 10^{6} \mathrm{~cm}^{-3}$, regardless of dynamical evolutionary phases. The depletion of sulfur-bearing molecules such as CS, SO, and CCS is particularly sensitive to the density increase because they tend to stick to both $\mathrm{H}_{2} \mathrm{O}$ and $\mathrm{CO}$ ice covered grains. In contrast, nitrogen-bearing molecules of $\mathrm{NH}_{3}$ and $\mathrm{N}_{2} \mathrm{H}^{+}$do not seem to be susceptible to freezing out on the grain surface (Bergin \& Langer 1997). This prediction is confirmed by the recent observations toward starless cores, e.g., Tafalla et al. (2002), who showed that $\mathrm{N}_{2} \mathrm{H}^{+}$and $\mathrm{NH}_{3}$ column densities correlate better with the dust column density than molecules such as $\mathrm{CO}$ and $\mathrm{CS}$. This characteristic, coupled with high angular resolution, makes $\mathrm{N}_{2} \mathrm{H}^{+}$ideal as a reference molecule. In addition, we believe that the possible low-velocity compact outflow does not affect our discussion about molecular abundances, considering our findings from radial density profile, position-velocity (PV) diagrams, and velocity width maps of the core (described in $\S \S 4.4$ and 4.5).

\subsection{1. $\mathrm{N}_{2} \mathrm{H}^{+}$and $\mathrm{H}^{13} \mathrm{CO}^{+}$}

The abundances of $\mathrm{N}_{2} \mathrm{H}^{+}$and $\mathrm{H}^{13} \mathrm{CO}^{+}$are estimated through comparison to the mean $\mathrm{H}_{2}$ column density, $N\left(\mathrm{H}_{2}\right)_{8^{\prime \prime}}$, derived from the $350 \mu \mathrm{m}$ continuum flux with the SHARC II beam ( $\$ 3.1 .2)$. Because the extent of the $350 \mu \mathrm{m}$ emission is approximately 4 times smaller than those of the $\mathrm{N}_{2} \mathrm{H}^{+}$- and $\mathrm{H}^{13} \mathrm{CO}^{+}$-emitting regions, such a comparison is possible only in the common innermost region of the core. Comparing the $N\left(\mathrm{H}_{2}\right)_{8^{\prime \prime}}$ with the $\mathrm{N}_{2} \mathrm{H}^{+}$ and $\mathrm{H}^{13} \mathrm{CO}^{+}$column densities with the SHARC II beam size (Table 6), we obtained $X\left(\mathrm{~N}_{2} \mathrm{H}^{+}\right)_{8^{\prime \prime}}=(9.5 \pm 5.0) \times 10^{-10}$ and $X\left(\mathrm{H}^{13} \mathrm{CO}^{+}\right)_{8^{\prime \prime}}=(8.5 \pm 4.7) \times 10^{-12}$ (Table 7). Note that the errors in $X_{m}$ are mostly due to the uncertainties in $\kappa_{\nu}$ (§ 3.1.2).

TABLE 7

Fractional Abundances of Dense Gas Tracer Molecules

\begin{tabular}{|c|c|c|c|}
\hline Molecule & $X_{m}{ }^{\mathrm{a}}$ & $\begin{array}{c}\text { Beam Size } \\
(\operatorname{arcsec})\end{array}$ & Comments \\
\hline $\mathrm{N}_{2} \mathrm{H}^{+} \ldots \ldots \ldots \ldots \ldots \ldots \ldots \ldots \ldots$ & $(9.5 \pm 5.0) \mathrm{E}-10$ & $9 \times 8$ & Used $\left\langle N\left(\mathrm{H}_{2}\right)\right\rangle^{\mathrm{b}}$ in $\S 3.1 .2$ \\
\hline $\mathrm{H}^{13} \mathrm{CO}^{+} \ldots \ldots \ldots \ldots \ldots \ldots \ldots$ & $(8.5 \pm 4.7) \mathrm{E}-12$ & $9 \times 8$ & Used $\left\langle N\left(\mathrm{H}_{2}\right)\right\rangle^{\mathrm{b}}$ in $\S 3.1 .2$ \\
\hline $\mathrm{C}_{3} \mathrm{H}_{2} \ldots \ldots \ldots \ldots \ldots \ldots \ldots \ldots \ldots \ldots \ldots$ & $(2 \pm 1) \mathrm{E}-10$ & 20 & $X\left(\mathrm{~N}_{2} \mathrm{H}^{+}\right)_{8^{\prime \prime}} \times\left(N\left[\mathrm{C}_{2} \mathrm{H}_{2}\right] / N\left[\mathrm{~N}_{2} \mathrm{H}^{+}\right]\right)\left(20^{\prime \prime}\right)^{\mathrm{c}}$ \\
\hline CCS & $(5 \pm 3) \mathrm{E}-10$ & 40 & $X\left(\mathrm{~N}_{2} \mathrm{H}^{+}\right)_{8^{\prime \prime}} \times\left(N[\mathrm{CCS}] / N\left[\mathrm{~N}_{2} \mathrm{H}^{+}\right]\right)\left(40^{\prime \prime}\right)^{\mathrm{c}}$ \\
\hline 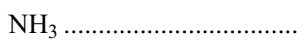 & $(2 \pm 1) \mathrm{E}-8$ & 80 & $X\left(\mathrm{~N}_{2} \mathrm{H}^{+}\right)_{8^{\prime \prime}} \times\left(N\left[\mathrm{NH}_{3}\right] / N\left[\mathrm{~N}_{2} \mathrm{H}^{+}\right]\right)\left(80^{\prime \prime}\right)^{\mathrm{c}}$ \\
\hline
\end{tabular}

\footnotetext{
${ }^{\text {a }}$ Fractional abundance of the molecule.

b We adopted mean $\mathrm{H}_{2}$ column density, $\left\langle N\left(\mathrm{H}_{2}\right)\right\rangle$, from cases I and II in $\S 3.1 .2$ and considered the range of $\mathrm{H}_{2}$ column densities into the errors in $X_{m}$.

${ }^{c}$ Assumed that $\mathrm{N}_{2} \mathrm{H}^{+}$does not have radial abundance variation. Column density ratios are calculated from Table 6 . The notation of $X\left(\mathrm{~N}_{2} \mathrm{H}^{+}\right)_{8^{\prime \prime}}$ indicates $\mathrm{N}_{2} \mathrm{H}^{+}$abundance measured with the SHARC II beam.
} 
TABLE 8

LTE and Virial Masses of GF 9-2 Core

\begin{tabular}{|c|c|c|c|c|c|}
\hline \multirow[b]{2}{*}{ LiNE } & \multirow[b]{2}{*}{$\begin{array}{c}\mathcal{A}^{\mathrm{b}} \\
\left(\mathrm{pc}^{2}\right)\end{array}$} & \multirow[b]{2}{*}{$\begin{array}{c}M_{\mathrm{LTE}}{ }^{\mathrm{c}} \\
\left(M_{\odot}\right)\end{array}$} & \multirow[b]{2}{*}{$\begin{array}{l}R_{\text {eff }}{ }^{\mathrm{d}} \\
(\mathrm{pc})\end{array}$} & \multicolumn{2}{|c|}{$M_{\mathrm{vir}}^{\mathrm{a}}$} \\
\hline & & & & $\begin{array}{c}\text { Case } \mathrm{I}^{\mathrm{e}} \\
\left(M_{\odot}\right)\end{array}$ & $\begin{array}{c}\text { Case } \mathrm{II}^{\mathrm{e}} \\
\left(M_{\odot}\right)\end{array}$ \\
\hline $\mathrm{N}_{2} \mathrm{H}^{+}(1-0) \ldots \ldots \ldots \ldots \ldots$ & $2.632 \mathrm{E}-3$ & $1.2 \pm 0.7$ & 0.0289 & $0.39 \pm 0.3$ & $0.10 \pm 0.08$ \\
\hline $\mathrm{H}^{13} \mathrm{CO}^{+}(1-0) \ldots \ldots$. & $3.963 \mathrm{E}-3$ & $1.3 \pm 0.3$ & 0.0354 & $2.9 \pm 1.7$ & $0.12 \pm 0.07$ \\
\hline $\mathrm{CCS} 4_{3}-3_{2} \ldots \ldots \ldots \ldots$ & $5.897 \mathrm{E}-3$ & $3.1 \pm 1.9$ & 0.0432 & $1.7 \pm 1.4$ & $0.078 \pm 0.06$ \\
\hline $\mathrm{NH}_{3}(1,1) \ldots \ldots \ldots \ldots$ & $5.544 \mathrm{E}-3$ & $4.5 \pm 2.4$ & 0.0419 & $2.3 \pm 1.6$ & $0.25 \pm 0.15$ \\
\hline
\end{tabular}

${ }^{\text {a }}$ Errors in virial mass are calculated from $\left(\delta M_{\text {vir }}\right)^{2}=\left(\partial M_{\text {vir }} /\right.$ $\left.\partial\left\langle\Delta v_{\text {int }}\right\rangle\right)^{2}\left(\delta\left\langle\Delta v_{\text {int }}\right\rangle\right)^{2}+\left(\partial M_{\text {vir }} / \partial R_{\text {eff }}\right)^{2}\left(\delta R_{\text {eff }}\right)^{2}$, where $\delta R_{\text {eff }}$ and $\delta\left\langle\Delta v_{\text {int }}\right\rangle$ are those in $R_{\text {eff }}$ and $\left\langle\Delta v_{\text {int }}\right\rangle$, respectively. The intrinsic velocity width $\left(\Delta v_{\text {int }}\right)$ and its error are taken from those for the entire core in Table 5.

b Area enclosed by the $50 \%$ level contour of each total integrated intensity map (Fig. 4).

${ }^{c}$ Errors in LTE mass include those in the column density, area size, and the abundance.

${ }^{\mathrm{d}}$ Effective radius defined by $R_{\mathrm{eff}}=(\mathcal{A} / \pi)^{1 / 2}$.

e Cases I and II are, respectively, when $\left\langle\Delta v_{\text {int }}\right\rangle^{2}=\left\langle\Delta v_{\text {thm }}\right\rangle^{2}+\left\langle\Delta v_{\text {turb }}\right\rangle^{2}$ and $\left\langle\Delta v_{\text {int }}\right\rangle^{2}=\left\langle\Delta v_{\text {thm }}\right\rangle^{2}$. Here $\left\langle\Delta v_{\text {int }}\right\rangle,\left\langle\Delta v_{\text {thm }}\right\rangle$, and $\left\langle\Delta v_{\text {turb }}\right\rangle$ are means of intrinsic, thermal, and turbulent velocity widths, respectively (see $\S 5.2 .1$ )

We argue that $\mathrm{N}_{2} \mathrm{H}^{+}$in GF 9-2 is not depleted, whereas $\mathrm{H}^{13} \mathrm{CO}^{+}$ is depleted. Our estimate of $X\left(\mathrm{~N}_{2} \mathrm{H}^{+}\right)_{8^{\prime \prime}}$ is comparable to those in the following studies. Womack et al. (1992) obtained a typical value of $X\left(\mathrm{~N}_{2} \mathrm{H}^{+}\right)=7 \times 10^{-10}$ in the dark cloud cores TMC-1 and L134N $\left(\theta_{\mathrm{HPBW}}=70^{\prime \prime}\right)$. Benson et al. (1998) derived $(7 \pm$ $4) \times 10^{-10}$ from a Haystack $37 \mathrm{~m}\left(22^{\prime \prime}\right)$ survey toward 60 lowmass cores. Caselli et al. (2002) reported $(3 \pm 2) \times 10^{-10}$ from

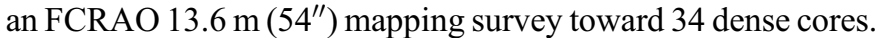
Tafalla et al. (2002) reported the range of $(0.75-2.4) \times 10^{-10}$ toward five starless cores using the FCRAO. The consistency between our measurements with an $8^{\prime \prime}$ beam and those with $22^{\prime \prime}$, $54^{\prime \prime}$, and $70^{\prime \prime}$ beams suggests that $\mathrm{N}_{2} \mathrm{H}^{+}$in GF 9-2 is not depleted at the core center. On the other hand, $X\left(\mathrm{H}^{13} \mathrm{CO}^{+}\right)_{8^{\prime \prime}}$ in GF 9-2 is by a factor of almost 6 smaller than the lowest abundances in other similar cores measured with a $67^{\prime \prime}$ beam (Table 9 of Butner et al. 1995), e.g., $5.6 \times 10^{-11}$ for B335 (Class 0) and $5.4 \times 10^{-11}$ for $\mathrm{L} 1495$ (Class I). This fact suggests that $\mathrm{H}^{13} \mathrm{CO}^{+}$in GF 9-2 is very likely to be depleted at the center, although we need to verify the radial variation of $X\left(\mathrm{H}^{13} \mathrm{CO}^{+}\right)$.

\subsection{2. $\mathrm{C}_{3} \mathrm{H}_{2}, \mathrm{CCS}$, and $\mathrm{NH}_{3}$}

Using the $X\left(\mathrm{~N}_{2} \mathrm{H}^{+}\right)_{8^{\prime \prime}}$ as a reference value, we estimated the abundances of $\mathrm{C}_{3} \mathrm{H}_{2}, \mathrm{CCS}$, and $\mathrm{NH}_{3}$. For this purpose, we assumed that $\mathrm{N}_{2} \mathrm{H}^{+}$in GF 9-2 does not have radial abundance variation. Recall that Tafalla et al. (2002) reported that $\mathrm{N}_{2} \mathrm{H}^{+}$abundances are spatially constant in the five starless cores they surveyed. Given the $X\left(\mathrm{~N}_{2} \mathrm{H}^{+}\right)_{8 \prime}$ and a column density ratio of $N\left(\mathrm{C}_{3} \mathrm{H}_{2}\right)$ / $N\left(\mathrm{~N}_{2} \mathrm{H}^{+}\right)$with a $20^{\prime \prime}$ beam (Table 6), we obtained $X\left(\mathrm{C}_{3} \mathrm{H}_{2}\right)_{20^{\prime \prime}}$ of $(2 \pm 1) \times 10^{-10}$ (Table 7). Similarly, we obtained $X(\mathrm{CCS})_{40^{\prime \prime}}$ of $(5 \pm 3) \times 10^{-10}$ from an $N(\mathrm{CCS}) / N\left(\mathrm{~N}_{2} \mathrm{H}^{+}\right)$ratio for $r \leq 20^{\prime \prime}$ and $X\left(\mathrm{NH}_{3}\right)_{80^{\prime \prime}}$ of $(2 \pm 1) \times 10^{-8}$ from an $N\left(\mathrm{NH}_{3}\right) / N\left(\mathrm{~N}_{2} \mathrm{H}^{+}\right)$ratio for $r \leq 40^{\prime \prime}$.

The $X\left(\mathrm{C}_{3} \mathrm{H}_{2}\right)_{20}$ " value in GF 9-2 would fall at the lower end of the $X\left(\mathrm{C}_{3} \mathrm{H}_{2}\right)$ range, $(3.2-40) \times 10^{-10}$, obtained from the survey toward dense dark clouds with a $54^{\prime \prime}$ beam (Cox et al. 1989), although the beam sizes differ by a factor of $\sim 2.5$. The estimated $X(\mathrm{CCS})_{40^{\prime \prime}}=(5 \pm 3) \times 10^{-10}$ is consistent with those of the starless cores measured with comparable beam sizes, i.e., $5.8 \times 10^{-10}$ in TMC-1D and $4.9 \times 10^{-10}$ in L1498 with $45^{\prime \prime}-53^{\prime \prime}$ beams (Wolkovitch et al. 1997), although smaller than $(8 \pm 3) \times 10^{-9}$

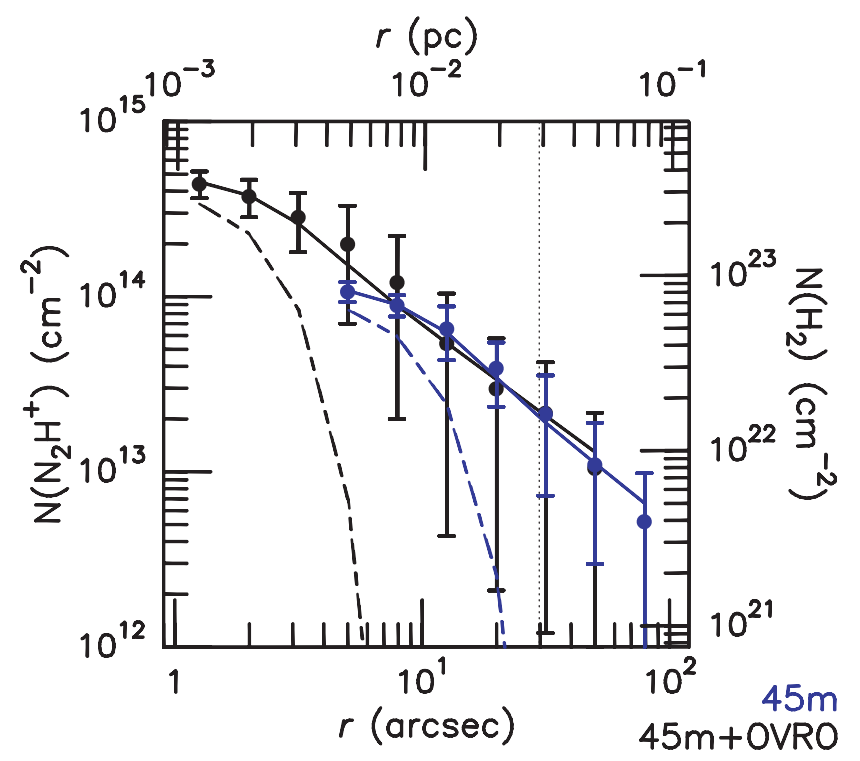

FIG. 11.- Radial column density profiles of the $\mathrm{N}_{2} \mathrm{H}^{+}$in the GF 9-2 core obtained from the Nobeyama $45 \mathrm{~m}$ telescope (blue) and the combined OVRO plus $45 \mathrm{~m}$ data (black) (see text). The upper horizontal axis shows the linear scale. The right-hand vertical axis represents $\mathrm{H}_{2}$ column density converted with $X\left(\mathrm{~N}_{2} \mathrm{H}^{+}\right)=$ $9.5 \times 10^{-10}$ ( Table 7). The solid lines indicate the best-fit power-law profiles ( $\left.\S 4.4\right)$ : $N_{\mathrm{N}_{2} \mathrm{H}^{+}}(r)=(7.3 \pm 1.1) \times 10^{14}\left(r / 1^{\prime \prime} .0\right)^{-(1.0 \pm 0.1)} \mathrm{cm}^{-2}$ for $r \leq 50^{\prime \prime}$ for the combined and $(8.5 \pm 2.5) \times 10^{14}\left(r / 1^{\prime \prime} .0\right)^{-(1.1 \pm 0.2)} \mathrm{cm}^{-2}$ for $r \leq 80^{\prime \prime}$ for the $45 \mathrm{~m}$ data. The dashed lines indicate the beam patterns: 17".2 in HPBW for the $45 \mathrm{~m}$ telescope, and 4". 1 for the combined data. The vertical dotted line at $r=29.9$ (corresponding to $0.029 \mathrm{pc}$ ) indicate the $R_{\text {eff }}$ for the $\mathrm{N}_{2} \mathrm{H}^{+}$core (Table 8).

with a $\sim 52^{\prime \prime}$ beam in L1521F (Shinnaga et al. 2004). On the other hand, the $X(\mathrm{CCS})_{40^{\prime \prime}}$ in GF 9-2 is found to be larger than those in the representative Class 0 sources B1 and B335 $\left(\simeq 9 \times 10^{-11}\right.$ and $\simeq 1.6 \times 10^{-10}$, respectively) reported in Lai \& Crutcher (2000) with the $\simeq 30^{\prime \prime}$ BIMA beam. Lastly, the $X\left(\mathrm{NH}_{3}\right)_{80^{\prime \prime}}=(2 \pm 1) \times$ $10^{-8}$ in GF 9-2 is somewhat smaller than the mean value derived from the large compilation of literature toward the cores in the Taurus and Ophiucus regions $\left(4.0_{-0.8}^{+2.3} \times 10^{-8}\right.$; Table B10 of Jijina et al. 1999) but is comparable to the range of $(0.4-2.3) \times 10^{-8}$ for the five starless cores surveyed by Tafalla et al. (2002).

Here we wish to discuss the age of the GF 9-2 core in terms of chemical evolution. Suzuki et al. (1992) analyzed their $45 \mathrm{~m}$ telescope CCS survey data by developing a chemical evolutionary model and concluded that CCS is the most abundant in the early evolutionary stage for a quiescent dark cloud core, whereas $\mathrm{NH}_{3}$ is less abundant and becomes abundant in the later evolutionary stage. Aikawa et al. (2001) advanced such a model for a dynamically collapsing core and pointed out that $\mathrm{N}_{2} \mathrm{H}^{+}$shows similar chemical evolution as $\mathrm{NH}_{3}$. Comparing the $X\left(\mathrm{NH}_{3}\right)_{80}$ " and $X(\mathrm{CCS})_{40^{\prime \prime}}$ with Figure 16 of Suzuki et al. (1992) in conjunction with Figure 5 of Aikawa et al. (2001), we argue that the GF 9-2 core has experienced $10^{5}-10^{6}$ yr since the diffuse stage. Because of the large uncertainty in our abundance estimate, we have limited our discussion to the three molecules whose predicted abundance variation with time is large enough to compare with our results.

\subsection{LTE Mass of the Core}

Using the column densities of the $\mathrm{N}_{2} \mathrm{H}^{+}, \mathrm{H}^{13} \mathrm{CO}^{+}, \mathrm{CCS}$, and $\mathrm{NH}_{3}$ lines $(\S 4.1)$ and their fractional abundances $(\S 4.2)$, we now calculate the LTE mass $\left(M_{\mathrm{LTE}}\right)$ of the gas in the area $(\mathcal{A})$ enclosed by the $50 \%$ level contour of each total integrated intensity map of Figure 4 (see Table 8 ). The mass is given by $M_{\mathrm{LTE}}=$ $\mu m_{\mathrm{H}}\left(N_{m} / X_{m}\right) \mathcal{A}$, where $\mu$ is the mean molecular weight (2.33 for 

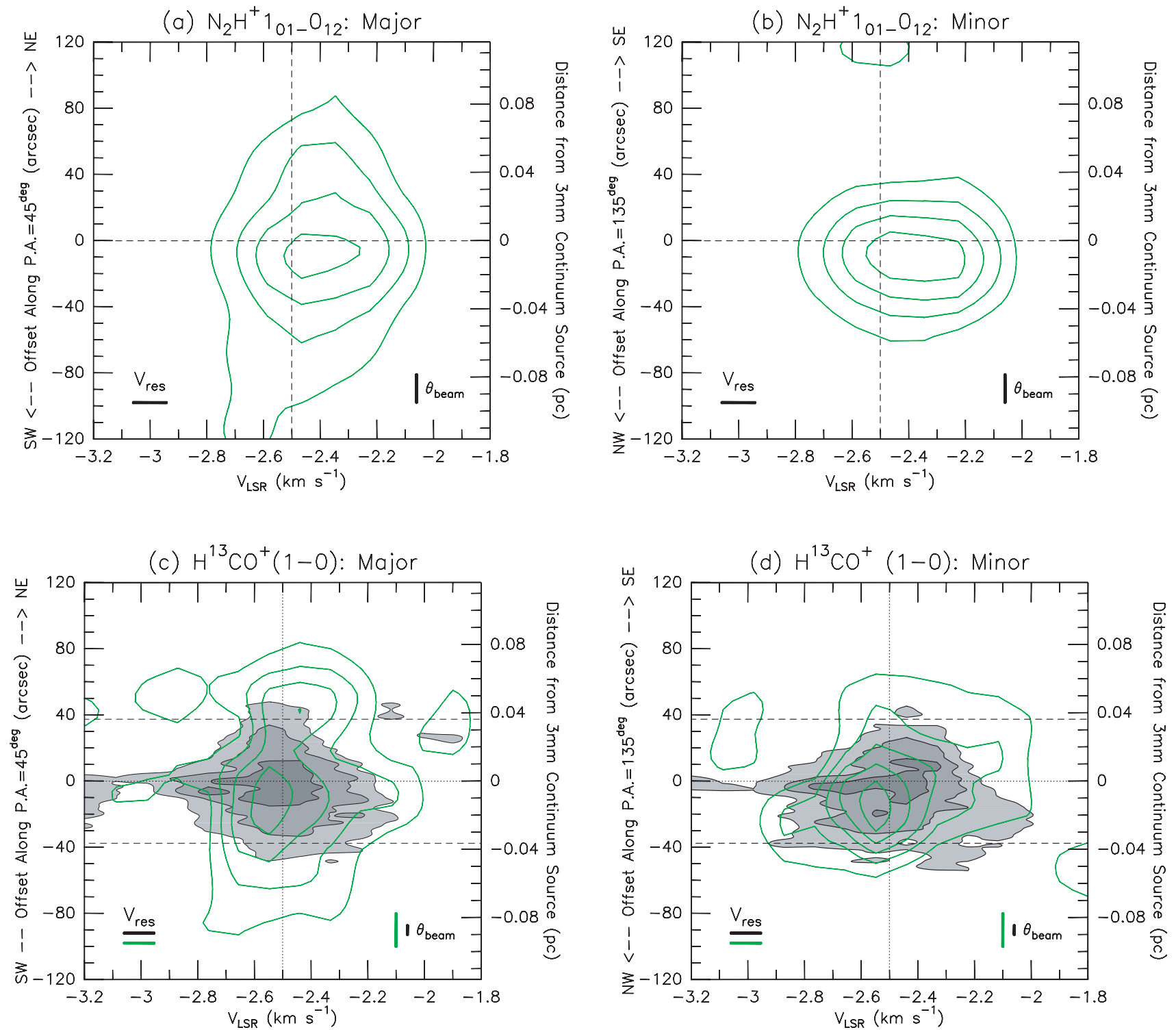

Fig. 12.- $\mathrm{PV}$ diagrams of the $\mathrm{N}_{2} \mathrm{H}^{+}(1-0)\left(F_{1}, F\right)=(0,1)-(1,2)$ (top) and $\mathrm{H}^{13} \mathrm{CO}^{+}(1-0)($ bottom) emission for the GF 9-2 core made from the $45 \mathrm{~m}$ telescope $($ green contour) and the combined data (gray scale with black contour). The left and right panels, respectively, show the diagrams along the major $\left(\mathrm{P} . \mathrm{A} .=45^{\circ}\right)$ and minor $\left(\right.$ P.A. $\left.=135^{\circ}\right)$ axes, passing through the continuum source. The right-hand vertical axis in each panel shows distance from the $3 \mathrm{~mm}$ source in pc. The contour intervals are the same as in Fig. 4. The intensity is presented in the $T_{\mathrm{mb}}$ scale for the $45 \mathrm{~m}$ data and in $S_{\nu}$ for the combined data. See the caption of Fig. 6 for the rms noise levels. The vertical and horizontal dotted lines indicate $V_{\text {sys }}=-2.5 \mathrm{~km} \mathrm{~s}^{-1}$ and the position of the $3 \mathrm{~mm}$ source, respectively. The horizontal dashed lines in $(c)$ and $(d)$ running at $\pm 37^{\prime \prime} 5$ indicate the FOV of the combined data. The horizontal and vertical bars labeled with $V_{\text {res }}$ and $\theta_{\text {beam }}$ indicate the velocity resolution and beam size, respectively.

$[\mathrm{He}]=0.1[\mathrm{H}])$ and $m_{\mathrm{H}}$ is the atomic mass of hydrogen. The obtained LTE masses range from 1.2 to $4.5 M_{\odot}$.

\subsection{Radial Column Density Profile of the Core}

We derive the radial column density profile of the GF 9-2 core using the $\mathrm{N}_{2} \mathrm{H}^{+}$column density maps. Recall that the $\mathrm{N}_{2} \mathrm{H}^{+}$in GF 9-2 is unlikely to be depleted over the full extent of the core, whereas the $\mathrm{H}^{13} \mathrm{CO}^{+}$may be depleted $(\S 4.2 .1)$. We assume that the core is spherical. To azimuthally average the column density maps, we take the $\mathrm{N}_{2} \mathrm{H}^{+}$emission peak as the center. Figure 11 represents the resultant column density profiles. The profiles can be well fitted by a power-law function of $N(r)=N_{0}\left(r / r_{0}\right)^{-p}$, where $p$ is the power-law index and $N_{0}$ is the column density at $r=r_{0}=1^{\prime \prime}$. By minimizing the $\chi^{2}$ values, we obtained the best-fit parameters of $p=1.0 \pm 0.1$ and $N_{0}=(7.3 \pm 1.1) \times 10^{14} \mathrm{~cm}^{-2}$ for the combined data and $p=1.1 \pm 0.2$ and $N_{0}=(8.5 \pm 2.5) \times$ $10^{14} \mathrm{~cm}^{-2}$ for the $45 \mathrm{~m}$ telescope data. The two fitting results show good consistency within uncertainties, indicating that a negligible amount of the dense gas has been evacuated by the possible compact outflow in the innermost region of $r \lesssim 3^{\prime \prime}$.

\subsection{Velocity Structure of the Core}

\subsubsection{Overall Velocity Structure}

To examine the velocity structure of the GF 9-2 core, we made $\mathrm{PV}$ diagrams using the $45 \mathrm{~m} \mathrm{~N}_{2} \mathrm{H}^{+}(1-0)\left(F_{1}, F\right)=(0,1)-(1,2)$ and $\mathrm{H}^{13} \mathrm{CO}^{+}(1-0)$ line data (Fig. 12). We also overlaid PV diagrams made from the combined $\mathrm{H}^{13} \mathrm{CO}^{+}$data set. Since the core is elongated along the northeast-southwest (Figs. 4, 5, and 9), we select the two cutting axes at P.A. of $45^{\circ}$ and $135^{\circ}$, passing through the position of the $3 \mathrm{~mm}$ source as the major and minor axes, respectively. Along the major axis, the $45 \mathrm{~m}$ data show a weak velocity gradient from northeast toward southwest in both molecular lines. The gradient along the major axis is of the order of $1 \mathrm{~km} \mathrm{~s}^{-1} \mathrm{pc}^{-1}$, comparable to the typical value measured in the Taurus dark cloud cores (Goodman et al. 1993). If the gradient 


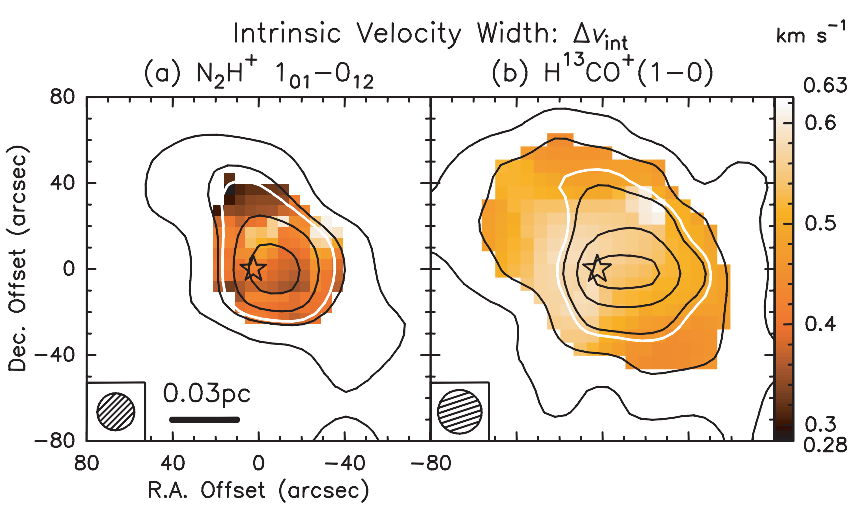

FIG. 13. - Intrinsic velocity width $\left(\Delta v_{\text {int }}\right)$ maps (color scale) of $(a) \mathrm{N}_{2} \mathrm{H}^{+}(1-0)$ $\left(F_{1}, F\right)=(0,1)-(1,2)$ and $(b) \mathrm{H}^{13} \mathrm{CO}^{+}(1-0)$ emission for the $45 \mathrm{~m}$ telescope data (§ 4.5.2). The presented $\Delta v_{\text {int }}$ ranges are $0.28 \leq \Delta v_{\text {int }} / \mathrm{km} \mathrm{s}^{-1} \leq 0.61$ for the $\mathrm{N}_{2} \mathrm{H}^{+}$and $0.44 \leq \Delta v_{\text {int }} / \mathrm{km} \mathrm{s}^{-1} \leq 0.63$ for the $\mathrm{H}^{13} \mathrm{CO}^{+}$. Contours in $(a)$ and $(b)$ indicate the $\mathrm{N}_{2} \mathrm{H}^{+}$and $\mathrm{H}^{13} \mathrm{CO}^{+}$total integrated intensity maps shown in Fig. 6 . The thick white contour in each panel indicates the $50 \%$ level contour of the total maps. The star and hatched ellipse are the same as in Fig. 4.

represents the rigid-body-like rotation of the core, it is converted to an angular velocity of $\Lambda \simeq 3 \times 10^{-14} \mathrm{~s}^{-1}$. On the other hand, the minor-axis plots do not show a well-defined velocity gradient commonly seen in the two lines, although the $\mathrm{H}^{13} \mathrm{CO}^{+}$diagram appears to show a southeast-northwest gradient if we concentrate on the lowest two contours.

Another important finding from Figure 12 is the presence of a broader line width region at the core center, prominently seen for the $\mathrm{H}^{13} \mathrm{CO}^{+}$. The blueshifted gas is seen up to $\simeq 0.6 \mathrm{~km} \mathrm{~s}^{-1}$ from the $V_{\text {sys }}$ at the central region of $r \lesssim 5^{\prime \prime}$ (corresponding to $1000 \mathrm{AU}$ ), whereas the redshifted counterpart is $\simeq 0.3 \mathrm{~km} \mathrm{~s}^{-1}$. The presence of such blueshifted emission and the weak redshifted counterpart at the core center (see also Fig. 2) suggests that gas is infalling where redshifted gas is obscured by the foreground cold gas along the line of sight (e.g., Walker et al. 1986). Notice that the line broadening at the core center is unlikely to be caused by the possible compact outflow because the red lobe is more intense than the blue one (see Fig. 8), inconsistent with the above results.

\subsubsection{Velocity Width}

We first estimate intrinsic velocity width $\left(\Delta v_{\text {int }}\right)$ toward the core center by analyzing the $\mathrm{N}_{2} \mathrm{H}^{+}, \mathrm{H}^{13} \mathrm{CO}^{+}, \mathrm{C}_{3} \mathrm{H}_{2}, \mathrm{CCS}$, and $\mathrm{NH}_{3}$ lines (Table 5). Note that $\Delta v_{\text {int }}$ is the velocity width corrected for line broadening due to instrumental velocity resolution and optical depth of the line (Phillips et al. 1979). Both corrections are made for the $\mathrm{C}_{3} \mathrm{H}_{2}$ and CCS lines. The latter correction is not made for $\mathrm{N}_{2} \mathrm{H}^{+}$and $\mathrm{NH}_{3}$ data because HFS analysis obtains the optical depth-free $\Delta v_{\text {FWHM }}$ (see Appendix B), and not for the $\mathrm{H}^{13} \mathrm{CO}^{+}$data because it is optically thin. The obtained $\Delta v_{\text {int }}$ toward the center is between 0.35 and $0.61 \mathrm{~km} \mathrm{~s}^{-1}$. This range is 2-3.5 times larger than the isothermal sound speed $\left(c_{S}\right)$ of the ambient gas of $0.18 \mathrm{~km} \mathrm{~s}^{-1}$ at $T_{k}=9.5 \mathrm{~K}(\S 4.1 .1)$.

Subsequently, we analyzed the velocity width structure of the core. Figure 13 shows the $\Delta v_{\text {int }}$ maps made by spectral moment analysis of the $45 \mathrm{~m}$ telescope $\mathrm{N}_{2} \mathrm{H}^{+}(1-0)\left(F_{1}, F\right)=(0,1)-$ $(1,2)$ and $\mathrm{H}^{13} \mathrm{CO}^{+}(1-0)$ line data. We adopt the moment method to keep a consistency in the analysis between the two lines. To make the maps, we made spectra from the cube data at each point, then searched for a peak LSR velocity in each spectrum to determine two velocity ranges from the peak velocity toward both the blue- and redshifted sides where the intensities are above the $1.5 \sigma$ level. We calculated the second-order spectral moments over the velocity ranges defined for the individual spectra. For the $\mathrm{N}_{2} \mathrm{H}^{+}$ map (Fig. 13b), we corrected the line broadening effect due to optical depth. We used equation (3) of Phillips et al. (1979) by substituting $\tau_{p}$ of equation (3) with $\tau_{\text {tot }} \times s$, where $s$ is the intrinsic relative intensity of the $\left(F_{1}, F\right)=(0,1)-(1,2)$ transition $(0.111$; Cazzoli et al. 1985). The maximum line broadening at the most opaque $\left(\tau_{\text {tot }} \times 0.111=1.5\right)$ position is 1.3 times the width for $\tau_{p} \rightarrow 0$. Considering the thermal noise and the velocity resolutions, we estimate uncertainties of 0.11 and $0.12 \mathrm{~km} \mathrm{~s}^{-1}$ in $\Delta v_{\text {int }}$ for $\mathrm{N}_{2} \mathrm{H}^{+}$and $\mathrm{H}^{13} \mathrm{CO}^{+}$, respectively. As seen in $\mathrm{PV}$ diagrams ( $\$$ 4.5.1), both the $\mathrm{N}_{2} \mathrm{H}^{+}$and $\mathrm{H}^{13} \mathrm{CO}^{+}$maps show a clear increase of $\Delta v_{\text {int }}$ toward the core center. The peak of the broadening does not fall onto the core center. It also should be noted that $\Delta v_{\text {int }}$ of the core gas exceeds $c_{s}$ of $0.18 \mathrm{~km} \mathrm{~s}^{-1}$ over the core (see also Table 5). Furthermore, comparing Figures 8 and 13, one cannot discern any clear effects by the possible compact outflow, such as an evacuated shell, in Figure 8; the ${ }^{12} \mathrm{CO}(3-2)$ condensations are only observed toward the $3 \mathrm{~mm}$ continuum source.

The above analysis clearly shows that there exist nonthermal motions in the core. We estimate nonthermal velocity width $\left(\Delta v_{\text {nth }}\right)$ by subtracting the thermal velocity width $\left(\Delta v_{\text {thm }}\right)$ from $\Delta v_{\text {int }}:\left(\Delta v_{\text {int }}\right)^{2}=\left(\Delta v_{\text {thm }}\right)^{2}+\left(\Delta v_{\text {nth }}\right)^{2}$. Here the thermal contribution is given by $\Delta v_{\text {thm }}=\left[8(\ln 2) k T_{k} / m_{\text {mol }}\right]^{1 / 2}$, where $k$ is Boltzmann's constant and $m_{\mathrm{mol}}$ is the molecular weight: $29 \mathrm{amu}$ for $\mathrm{N}_{2} \mathrm{H}^{+}, 30 \mathrm{amu}$ for $\mathrm{H}^{13} \mathrm{CO}^{+}, 56 \mathrm{amu}$ for $\mathrm{CCS}$, and $17 \mathrm{amu}$ for $\mathrm{NH}_{3}$. Assuming that $T_{k}$ is equal to $T_{\text {ex }}$ of $9.5 \mathrm{~K}(\S 4.1 .1)$ and that $T_{k}$ is constant over the entire core, $\Delta v_{\text {thm }}$ is calculated to be $0.12 \mathrm{~km} \mathrm{~s}^{-1}$ for $\mathrm{N}_{2} \mathrm{H}^{+}, 0.12 \mathrm{~km} \mathrm{~s}^{-1}$ for $\mathrm{H}^{13} \mathrm{CO}^{+}, 0.09 \mathrm{~km} \mathrm{~s}^{-1}$ for $\mathrm{CCS}$, and $0.16 \mathrm{~km} \mathrm{~s}^{-1}$ for $\mathrm{NH}_{3}$ lines. This leads to mean nonthermal velocity widths over the core of $\left\langle\Delta v_{\text {nth }}\right\rangle=0.21,0.58$, 0.40 , and $0.46 \mathrm{~km} \mathrm{~s}^{-1}$ for the $\mathrm{N}_{2} \mathrm{H}^{+}, \mathrm{H}^{13} \mathrm{CO}^{+}, \mathrm{CCS}$, and $\mathrm{NH}_{3}$ lines, respectively, suggesting the presence of supersonic gas motions. In obtaining $\left\langle\Delta v_{\text {nth }}\right\rangle$, we used the $\left\langle\Delta v_{\text {int }}\right\rangle$ values in Table 5.

\section{DISCUSSION}

\subsection{Evolutionary Stage of the GF 9-2 Core: A Very Young Protostar before Formation of a Large-Scale Molecular Outflow}

With its low luminosity $\left(L_{\text {bol }} \simeq 0.3 L_{\odot}\right)$ and cold SED $\left(T_{\text {bol }} \leq\right.$ $20 \mathrm{~K}$; Wiesemeyer 1997), we believe that the protostar embedded in the GF 9-2 core is at an extremely early phase of low-mass star formation, evidenced by the absence of a large-scale high-velocity molecular outflow, the possible detection of a compact low-velocity outflow, and the detection of $\mathrm{H}_{2} \mathrm{O}$ masers. We estimate the protostar's age from dynamical timescale $\left(t_{\text {dyn }}\right)$ of the possible compact low-velocity outflow ( $\S 3.3 .1)$. The terminal velocity $\left(V_{t}\right)$ of the spectra would give the line-of-sight component of the outflow velocity of $\left|V_{t}-V_{\text {sys }}\right|=V_{\text {flow }} \cos i$, where $i$ is the inclination angle of the outflow axis to the line of sight. Given the projected lobe length of $l_{\text {lobe }} \sin i=1.1 \times 10^{4}$ and $6.6 \times 10^{3} \mathrm{AU}$ and $V_{\text {flow }} \cos i \simeq 3.3$ and $3.1 \mathrm{~km} \mathrm{~s}^{-1}(\S 3.3 .1)$, we have $t_{\text {dyn }}=$ $l_{\text {lobe }} / V_{\text {flow }} \simeq 1.6 \times 10^{4}$ and $1.0 \times 10^{4} \mathrm{yr}$ with $i=45^{\circ}$ for the blueand redshifted ${ }^{12} \mathrm{CO}(3-2)$ lobes, respectively. These dynamical timescales are comparable to those for the extremely young Class 0 sources, e.g., $(0.6-3) \times 10^{4} \mathrm{yr}$ for VLA 1623 and $(0.8-2) \times 10^{4} \mathrm{yr}$ for IRAM 04191 (Table 1 of André et al. 1999 and references therein).

The youth of GF 9-2 is demonstrated in Figure 14, where we present a plot of $\mathrm{CO}$ outflow momentum rate $\left(F_{\mathrm{CO}}\right)$ normalized by the bolometric luminosity $\left(F_{\mathrm{CO}} c / L_{\mathrm{bol}}\right)$ versus $T_{\mathrm{bol}}$ for nearby $\left(d \leq 350\right.$ pc) low-mass $\left(L_{\text {bol }} \leq 50 L_{\odot}\right)$ YSOs. We employ $F_{\mathrm{CO}} c /$ $L_{\text {bol }}$ because molecular outflows in low-mass protostars are likely to be momentum driven (e.g., Cabrit \& Bertout 1992; Richer et al. 2000 and references therein). The temperature $T_{\text {bol }}$ is believed 


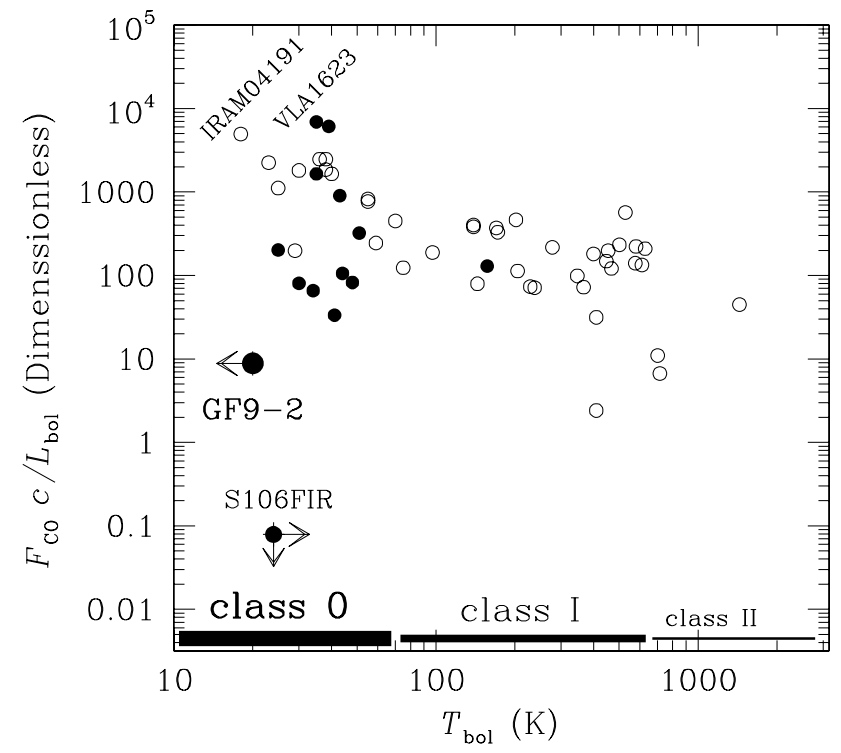

FIG. 14.-Plot of normalized CO outflow momentum flux $\left(F_{\mathrm{CO}} c / L_{\mathrm{bol}}\right)$ vs. bolometric temperature $\left(T_{\mathrm{bol}}\right)$ for nearby $(d<350 \mathrm{pc})$ low-mass YSOs less luminous than $50 L_{\odot}(\S 5.1)$, including S106 FIR $\left(L_{\text {bol }} \lesssim 1080 L_{\odot}\right.$; Richer et al. 1993 ; Furuya et al. 2000). The filled and open circles represent the presence and the absence of $\mathrm{H}_{2} \mathrm{O}$ masers in the period of the Nobeyama survey (Furuya et al. $2001,2003)$, respectively. The locus of GF 9-2 $\left(L_{\text {bol }} \simeq 0.3 L_{\odot}\right)$ is indicated by the large filled circle. The upper limit in $F_{\mathrm{CO}} c / L_{\mathrm{bol}}$ for S106 FIR is recalculated from Furuya et al. (2000) with the $3 \sigma$ upper limit and the outflow velocity of $50 \mathrm{~km} \mathrm{~s}^{-1}$. The $T_{\mathrm{bol}}$ is taken from Chen et al. (1995) and André et al. (2000), and $F_{\mathrm{CO}} c / L_{\mathrm{bol}}$ is calculated from Table 4 of Furuya et al. (2003). The $T_{\mathrm{bol}}$ ranges for the Class 0, I, and II sources (Chen et al. 1995) are shown by the bottom bars.

to trace the evolutionary stage of YSOs (Myers \& Ladd 1993). We calculate the $3 \sigma$ upper limit of $F_{\mathrm{CO}} c / L_{\text {bol }}=\left(M_{\text {lobe }} V_{\text {flow }}^{2} / l_{\text {lobe }}\right)$ $\left(c / L_{\text {bol }}\right) \simeq 9$ and 20 for the ${ }^{12} \mathrm{CO}(3-2)$ blue and red lobes, respectively, with $i=45^{\circ}$. Figure 14 illustrates that "Class 0 " is the most powerful outflow phase and "Class I" is the decline phase (e.g., Moriarty-Schieven et al. 1994). The most remarkable result is that GF 9-2 shows the lowest outflow activity, among the coldest low-mass Class 0 sources, although there exists enough circumstellar material ( $(4.3)$ to be dragged by a possible protostellar jet to develop a large-scale molecular outflow. Note that the prototypical Class 0 sources of VLA 1623 (André et al. 1993) and IRAM 04191 (André et al. 1999) have powerful CO outflows, despite that their bolometric luminosities are comparable to that of GF 9-2: $1 L_{\odot}$ for VLA 1623 and $0.15 L_{\odot}$ for IRAM 04191. We thus believe that the low luminosity of GF 9-2 is not responsible for the lowest outflow activity. Consequently, it is very likely that the protostar of our interest is the youngest Class 0 protostar ever identified and is just at the beginning of the outflow launch.

\subsection{The Initial Conditions for Gravitational Collapse of a Low-Mass Star-forming Core}

\subsubsection{Stability of the GF 9-2 Core}

If we interpret that the nonthermal velocity width obtained in the GF 9-2 core ( $\S 4.5 .2)$ is attributed to turbulent motions, we expect that the turbulence can help to support the core against its self-gravity. To test this hypothesis, we compare the LTE mass of the core with virial mass $M_{\mathrm{vir}}$ including the turbulence. Assuming a spherical system, the virial mass is given by $M_{\mathrm{vir}} \simeq$ $R_{\text {eff }}\left\langle\Delta v_{\text {int }}\right\rangle^{2} / G$, where $\left\langle\Delta v_{\text {int }}\right\rangle$ includes the nonthermal width, i.e., the turbulence. Given $\left\langle\Delta v_{\text {int }}\right\rangle$ and $R_{\text {eff }}$ values (Tables 5 and 8), we found that $M_{\mathrm{vir}}$ ranges from 0.39 to $2.9 M_{\odot}$ (case I in Table 8). Since $M_{\mathrm{vir}} \simeq M_{\mathrm{LTE}}$ within the uncertainties, the core is most likely to be in virial equilibrium. However, it should be noted that this hypothesis does not reconcile with the line broadening toward the core center (Fig. 13). Moreover, as far as we know, no cores that are supported by turbulence exhibit $\rho(r) \propto r^{-2}$ density profiles (see Fig. 11). Therefore, we conclude that the nonthermal line width is not caused by turbulence.

Alternatively, if we consider that infall causes the nonthermal line width, that is, if the GF 9-2 core is gravitationally unstable, the drawbacks in the previous hypothesis would be solved. The presence of infalling motion is supported by the following facts: (1) the presence of the blueshifted gas and the less prominent redshifted counterpart in the central $r \lesssim 1000 \mathrm{AU}(\S 4.5 .1)$, (2) the spectroscopic signature of infall, i.e., the "blue-skewed" profile observed in the optically thick $\mathrm{N}_{2} \mathrm{H}^{+}(1-0)\left(F_{1}, F\right)=(0,1)-$ $(1,2)$ line (Fig. 2), and (3) the detection of the $\mathrm{H}_{2} \mathrm{O}$ masers, a clear signpost of star formation, naturally leading to the presence of accretion flow onto the star-disk system. We, however, point out a drawback in item 2 above: the optically thin $\mathrm{H}^{13} \mathrm{CO}^{+}$emission does not show a single Gaussian profile expected from the infall interpretation, while the CCS does. In this interpretation, $M_{\text {vir }}$ should be estimated only from the thermal motion as $M_{\mathrm{vir}}=$ $\left(R_{\text {eff }} / G\right)\left(k T_{k} / \mu m_{\mathrm{H}}\right)$. The estimated $M_{\text {vir }}$ from the four lines takes a range between 0.078 and $0.25 M_{\odot}$ (case II in Table 8 ), which is 1 order of magnitude smaller than $M_{\mathrm{LTE}}$. The small $M_{\mathrm{vir}}$ is consistent with our interpretation that the core is under gravitational collapse. As discussed in $\S 4.5 .2$, the core would be rotating with $\Lambda \simeq 10^{-14} \mathrm{~s}^{-1}$. The collapse cannot be supposed by the centrifugal force due to the rotation because the rotational energy is less than a few percent of total energy required to support the core against its self-gravity. For such an unstable core, the gas is expected to be more perturbed toward the center, and the velocity width should increase inward as a result. In fact, Figure 13 clearly indicates that $\Delta v_{\text {int }}$ in GF $9-2$ increases up to $\simeq 0.6 \mathrm{~km} \mathrm{~s}^{-1}$ toward the center.

\subsubsection{Initial Conditions for the Gravitational Collapse of the Core}

To bring out the initial conditions for the gravitational collapse in the GF 9-2 core, we examine the density and velocity structures by comparing with some key characteristics of the Shu (1977) and Larson-Penston (Larson 1969; Penston 1969) solutions. The radial $\mathrm{H}_{2}$ column density profile of the core is given by $(7.7 \pm$ $4.1) \times 10^{23}\left[X\left(\mathrm{~N}_{2} \mathrm{H}^{+}\right) / 9.5 \times 10^{-10}\right]^{-1}(r / 200 \mathrm{AU})^{-(1.0 \pm 0.1)} \mathrm{cm}^{-2}$ for $0.001<r / \mathrm{pc}<0.05$ from the combined data and $(8.9 \pm$ $4.5) \times 10^{23}\left[X\left(\mathrm{~N}_{2} \mathrm{H}^{+}\right) / 9.5 \times 10^{-10}\right]^{-1}(r / 200 \mathrm{AU})^{-(1.1 \pm 0.2)} \mathrm{cm}^{-2}$ for $0.005<r / p c<0.08$ from the $45 \mathrm{~m}$ data (Fig. 11). It should be noted that the dense $\mathrm{N}_{2} \mathrm{H}^{+}$gas seems to obey the power-law profile with the index of -1 even at the innermost region of $r \lesssim$ 1000 AU where a possible compact outflow might begin to destroy the core. The power-law indices of -1.0 and -1.1 , converted into -2.0 and -2.1 for the volume density, well agree with those in the two solutions after the formation of the protostar (Shu 1977; Whitworth \& Summers 1985). The best-fit $\mathrm{H}_{2}$ column densities at $r=200$ AU fall between the two extreme predictions at $T=9.5 \mathrm{~K}:(2.8 \pm 1.5) \times 10^{23} \mathrm{~cm}^{-2}$ for the Shu solution and $(13 \pm 7) \times 10^{23} \mathrm{~cm}^{-2}$ for the Larson-Penston solution. Here we used a relation of $N_{0}\left(\mathrm{H}_{2}\right)=1.0$ and $4.4\left(c_{s}^{2} / 2 G r_{0}\right)\left[X\left(\mathrm{~N}_{2} \mathrm{H}^{+}\right) / \mu m_{\mathrm{H}}\right]$ for the Shu and Larson-Penston cases, respectively (Hunter 1977; Shu 1977; Foster \& Chevalier 1993). Because of the abundance uncertainty, however, we cannot conclude which solution describes well the collapse in the GF 9-2 core.

Contrary to the above discussion based on the density profile, the velocity width maps (Fig. 13) help to discriminate the two theoretical scenarios for the collapse of the GF 9-2 core. Notice 
that the influence of the compact outflow on the velocity width cannot be discerned toward the $3 \mathrm{~mm}$ source. Figure 13 shows that the velocity width decreases outward but remains $\simeq 0.3 \mathrm{~km} \mathrm{~s}^{-1}$, which is twice the ambient $c_{s}$, even in the outermost region of $r \simeq 50^{\prime \prime}$ (corresponding to $0.05 \mathrm{pc}$ ) where the density profile shows $\rho(r) \propto r^{-2}$ (Fig. 11). Recall that the $\rho(r) \propto r^{-2}$ profile characterizes the outer part of a collapsing core. These facts clearly indicate that the extended Larson-Penston solution for $t>0$ is more likely to be the case because it requires the infalling state when a protostar is about to be born $\left[v(r) \sim-3.28 c_{s}\right.$ at $t \rightarrow-0$; Hunter 1977], whereas the Shu solution requires a static state $[v(r)=0$ at $t=0]$. It is, therefore, highly probable that the gravitational collapse of the GF 9-2 core is well described by the runaway collapse scenario.

Subsequently, we estimate the age of the GF 9-2 protostar $\left(t_{\text {protostar }}\right)$ based on the lack of the density profile of $\rho(r) \propto r^{-3 / 2}$ down to the innermost region of $r \simeq 3^{\prime \prime}$ (see Fig. 11). Recall that the $\rho(r) \propto r^{-3 / 2}$ profile is expected for a central free-fall region that appears in both of the solutions at $t>0$. The nondetection of a free-fall density profile indicates that the central free-fall region, propagating outward with supersonic velocity, has not yet developed up to a radius $\left(r_{\mathrm{ff}}\right)$ of $\simeq 600 \mathrm{AU}$, suggestive of the youth of the GF 9-2 protostar. However, we do not exclude the possibility that the absence of a detection of the $\rho(r) \propto r^{-3 / 2}$ profile at the center could be caused by the formation of a binary (described in $\S 5.3$ ) whose projected separation is $\simeq 1200$ AU. Using equations (9) and (10) of Ogino et al. (1999), we estimate $t_{\text {protostar }}$ to be less than $t_{\text {protostar }} \simeq 5000(T / 9.5 \mathrm{~K})^{-1 / 2} \mathrm{yr}$ since the formation of the central protostar for the Larson-Penston solution. Although the age estimate is a factor of 2-3 smaller than the outflow dynamical timescale of $t_{\text {dyn }} \simeq(1.0-1.6) \times 10^{4} \mathrm{yr}(\S 5.1)$, we argue that the central protostar has just begun to exhibit its jet activity traced by the $\mathrm{H}_{2} \mathrm{O}$ masers $(\S 5.1)$.

Consequently, we propose that the GF 9-2 core has started its gravitational collapse with an initially unstable state. Here the term "initial " means $t=-t_{\mathrm{ff}}$, where $t_{\mathrm{ff}}$ is the free-fall time of the core (Larson 1969; Penston 1969). For GF 9-2, we calculate $t_{\mathrm{ff}} \simeq$ $2 \times 10^{5} \mathrm{yr}$. We therefore believe that the age of the core, $t_{\text {core }}=$ $t_{\text {ff }}+t_{\text {protostar }}$, would be $\simeq 2 \times 10^{5} \mathrm{yr}$, which has a consistency with the timescale of chemical evolution $(\S 4.2 .2)$.

\subsubsection{Infall Velocity and Mass Accretion Rate in the Core}

Assuming that $\Delta v_{\text {int }}$ represents a velocity difference of blueand redshifted infalling gas along the line of sight, let us discuss infall velocity $\left(v_{\text {inf }}\right)$ of the core gas. We found that $v_{\text {inf }}$ varies from 0.2 to $0.4 \mathrm{~km} \mathrm{~s}^{-1}$ using a relation of $v_{\text {inf }} \simeq \frac{1}{2} \mid v_{\text {inf, red }}-$ $v_{\text {inf,blue }} / \cos i \simeq \Delta v_{\text {int }} / 2 \cos i$ for the $\Delta v_{\text {int }}$ range of $0.28-0.63 \mathrm{~km} \mathrm{~s}^{-1}$ over the core (see Fig. 15) and $i=45^{\circ}$. We now calculate the mass infall rate from $d M / d t=4 \pi R_{\text {eff }}^{2} \mu m_{\mathrm{H}} n_{\mathrm{H}_{2}}\left(R_{\text {eff }}\right) v_{\text {inf }} \simeq$ $2 \pi R_{\text {eff }}\left[\mu m_{\mathrm{H}} N_{\mathrm{N}_{2} \mathrm{H}^{+}}\left(R_{\text {eff }}\right) / X\left(\mathrm{~N}_{2} \mathrm{H}^{+}\right)\right] v_{\text {inf }}$ for a sphere with the radius of $R_{\text {eff }}$. Here $n_{\mathrm{H}_{2}}\left(R_{\text {eff }}\right)$ is the volume density of a thin shell whose radius is $R_{\text {eff }}$ and is approximated as $N_{\mathrm{N}_{2} \mathrm{H}^{+}}\left(R_{\text {eff }}\right) /$ $2 R_{\text {eff }} X\left(\mathrm{~N}_{2} \mathrm{H}^{+}\right)$. Given $v_{\text {inf }}=0.29 \mathrm{~km} \mathrm{~s}^{-1}$ at $r \simeq R_{\text {eff }}$ from the $\mathrm{N}_{2} \mathrm{H}^{+} \Delta v_{\text {int }}$ map (Fig. 13), we have $d M / d t \simeq 3 \times 10^{-5} M_{\odot} \mathrm{yr}^{-1}$ with uncertainty of a factor of 1.6 for $R_{\text {eff }}$ in Table 8 , the column density profile ( $(4.4)$, and $X_{m}$ in Table 7 . The inferred mass accretion rate falls within the range between the predicted values of $1.5 \times 10^{-6}$ and $7.4 \times 10^{-5} M_{\odot} \mathrm{yr}^{-1}$ for the Shu and LarsonPenston solutions, respectively, at $10 \mathrm{~K}$. These accretion rates are calculated by $m_{0} c_{\text {iso }}^{3} / G$, where $m_{0}$ is the nondimensional factor of 0.975 and 46.92 for the Shu and Larson-Penston solutions, respectively (Hunter 1977). Our small mass accretion rate, compared to that of the Larson-Penston solution, is attributed to both the smaller column density and infall velocity.

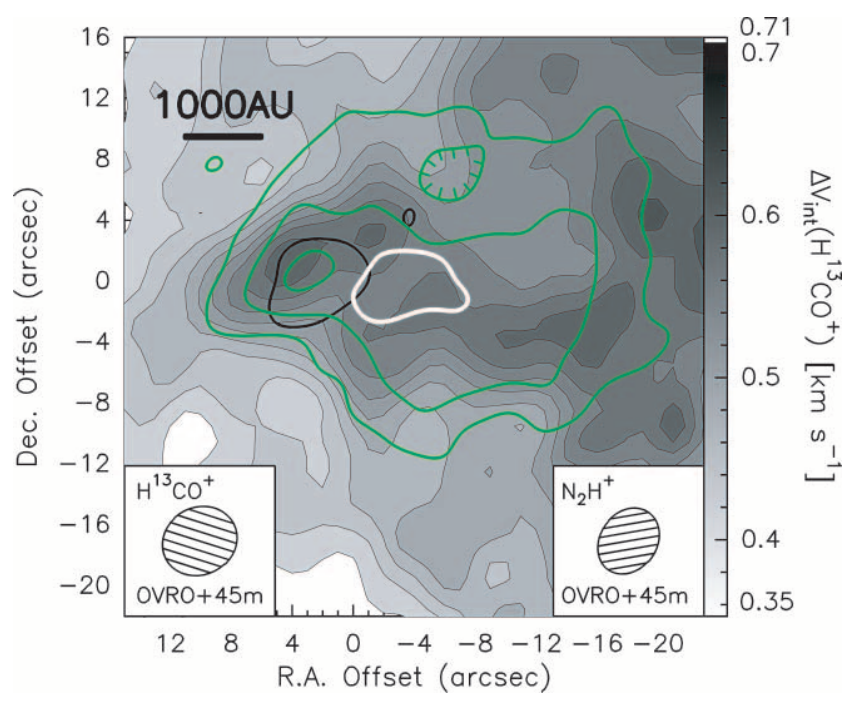

FIG. 15.-Map of the intrinsic velocity width of the $\mathrm{H}^{13} \mathrm{CO}^{+}(1-0)$ emission (gray scale and contour), obtained from the combined data. Contour intervals are the $10 \%$ step of the difference between the maximum $\left(0.71 \mathrm{~km} \mathrm{~s}^{-1}\right)$ and minimum $\left(0.36 \mathrm{~km} \mathrm{~s}^{-1}\right)$ velocity widths. The thick white and black contours show the $18 \sigma$ level of the total integrated intensity map of the combined $\mathrm{N}_{2} \mathrm{H}^{+}$ data (Fig. 6) and the $3 \sigma$ level of the $3 \mathrm{~mm}$ continuum emission (Fig. 1), respectively. The green contours show the highest three level contours $(18,21$, and $24 \sigma$ levels) of the total integrated intensity map of the combined $\mathrm{H}^{13} \mathrm{CO}^{+}$data (Fig. 6). The ellipses are the same as in Fig. 4.

\subsection{Is a Protobinary System Forming?}

We found that the position of the $3 \mathrm{~mm}$ continuum source is displaced by $\simeq 6^{\prime \prime}$ to the east of the $\mathrm{N}_{2} \mathrm{H}^{+}$column density peak, which would be explained by the presence of a protobinary system. In this interpretation, the $3 \mathrm{~mm}$ source represents a protostar and the $\mathrm{N}_{2} \mathrm{H}^{+}$peak a preprotostellar condensation. This is because the $\mathrm{N}_{2} \mathrm{H}^{+}$condensation is deeply embedded in the extended $350 \mu \mathrm{m}$ emission (Fig. $9 \mathrm{~b}$ ) but is not accompanied with a compact $3 \mathrm{~mm}$ source. To investigate the nature of the binary members, we made a $\Delta v_{\text {int }}$ map from the combined $\mathrm{H}^{13} \mathrm{CO}^{+}$data shown in Figure 15 as the line is expected to be optically thin. In $\S 3.1 .2$ we asserted that the $3 \mathrm{~mm}$ continuum emission would represent circumstellar material around a protostar. The line broadening toward the $3 \mathrm{~mm}$ source seen in Figures 12 and 15 suggests the local enhancement of mass accretion, leading to the existence of a protostar. On the other hand, the $\mathrm{N}_{2} \mathrm{H}^{+}$peak (white contour) seems to be a gas condensation or a preprotostellar core rather than an apparent peak caused by a local abundance enhancement because $X\left(\mathrm{~N}_{2} \mathrm{H}^{+}\right)_{8^{\prime \prime}}$ inferred over the $3 \sigma$ level contour of $350 \mu \mathrm{m}$ emission is comparable to those obtained with low spatial resolution studies $(\S 4.2 .1)$. It should be noted that the $\mathrm{H}^{13} \mathrm{CO}^{+}$ emission has a secondary peak near the $\mathrm{N}_{2} \mathrm{H}^{+}$condensation as seen in the OVRO maps (Fig. 6). Furthermore, the combined $\mathrm{N}_{2} \mathrm{H}^{+}$ map (Fig. 6) does not show a shell-like structure, which is expected to be seen if $\mathrm{N}_{2} \mathrm{H}^{+}$disappears from the gas phase at the core center (e.g., Kuiper et al. 1996; Belloche \& André 2004). We therefore conclude that the positional displacement of the $\mathrm{N}_{2} \mathrm{H}^{+}$peak is not caused by chemical evolution, but the $\mathrm{N}_{2} \mathrm{H}^{+}$peak represents a preprotostellar condensation, making it younger than the eastern object. The detection of the two objects reinforces the assertion that we witness a forming binary system with a separation of $\simeq 1200 \mathrm{AU}$.

The masses of the circumstellar material $\left(M_{\mathrm{cm}}\right)$ for each binary member would be on order of $0.1 M_{\odot}$. For the eastern object, the $3 \mathrm{~mm}$ continuum flux density ( $\S 3.1 .2$ ) integrated inside its $3 \sigma$ level contour (see Fig. 15) implies that the gas plus dust mass 
ranges from $\simeq 0.1$ (case $\mathrm{I}$ in $\S 3.1 .2)$ to $0.2 M_{\odot}$ (case II $)$. For the western object, we estimate $0.1-0.3 M_{\odot}$ from the $\mathrm{N}_{2} \mathrm{H}^{+}$intensity integrated inside the white contour, whose extent is similar to that of the $3 \mathrm{~mm}$ source (Fig. 15). The inferred $M_{\mathrm{cm}}$ seem reasonable for an amount of circumstellar material with $100 \mathrm{AU}$ scale around a newly formed protostar at the very early accretion phase because of $M_{\mathrm{cm}} \simeq M_{\text {protostar }}=(d M / d t) t_{\text {protostar }} \simeq 0.15 M_{\odot}(\S \S 5.2 .2$ and 5.2.3). In this context, the east-west elongated structure at the core center seen in the $45 \mathrm{~m}$ maps (Fig. 6) may be interpreted as a 1000 AU scale circumbinary disk-like structure because the size would be smaller than the extent of the protostellar envelope traced by the $350 \mu \mathrm{m}$ continuum emission $(6600 \times 4500 \mathrm{AU}$; $\S 3.1 .2)$. It is impossible to uniquely define the spatial extent of the disklike structure. However, if we arbitrarily define the disklike structure by a region enclosed by the 18 or $21 \sigma$ level contours of the combined $\mathrm{H}^{13} \mathrm{CO}^{+}$map (Fig. 15), mass of the circumbinary disk-like structure $\left(M_{\text {cbd }}\right)$ calculated from the $\mathrm{N}_{2} \mathrm{H}^{+}$data would be in the range $0.2-0.5 M_{\odot}$ with an approximate effective radius of $\simeq 2000$ AU. Considering the uncertainties in spatial extents and in $\kappa_{0}, \beta(\S 3.1 .2)$, and $X\left(\mathrm{~N}_{2} \mathrm{H}^{+}\right)(\S 4.2 .1)$, the mass of the disklike structure is likely to be plausible to be hosted in the $0.4-0.8 M_{\odot}$ envelope ( $\S 3.1 .2$ ) and to host the two binary members with the $0.1 M_{\odot}$ order circumstellar material. The mass distribution of $M_{\text {core }}>M_{\text {env }} \gtrsim M_{\text {cbd }} \gtrsim M_{\text {cm }}$ does not contradict our hypothesis that the central protostar(s) is at an extremely early evolutionary phase.

If a circumbinary disk exists, the radius of the disklike structure would be physically related to the rotation of the natal core, described in $\S 4.5$.1. Although the axis for the large-scale rotation of the core (P.A. $\left.\simeq 135^{\circ}\right)$ is not perpendicular to the major axis of the disklike structure (P.A. $\simeq 90^{\circ}$ ), a rough estimate of radius of the disklike structure would be given by the centrifugal radius, $R_{\text {cent }}$, calculated from the specific angular momentum of the core gas. With a relation of $R_{\text {cent }}=\left(4 R_{\text {eff }}^{4} \Lambda^{2}\right) /\left(G M_{\text {cbd }}\right)$, we obtained $R_{\text {cent }} \simeq 900 \mathrm{AU}$ for $\Lambda$ of $3 \times 10^{-14} \mathrm{~s}^{-1}(\S 4.5 .1)$ and $R_{\text {eff }}$ in Table 8 . The centrifugal radius is roughly twice the radius of the disklike structure, probably supporting our interpretation. Lastly, let us estimate the Toomre $Q$ parameter to examine whether or not gravitational fragmentation of the disklike structure led to the formation of the binary. The $Q$ parameter is given by $\Lambda c_{s} / \pi G \Sigma$ for Keplerian rotation. Here $\Sigma$ is the surface density of the disklike structure given by $M_{\mathrm{cbd}} / \mathcal{A}_{\mathrm{cbd}}$; an area of $\mathcal{A}_{\mathrm{cbd}} \simeq 4 \times 10^{33} \mathrm{~cm}^{2}$ is calculated for the region described above. We obtained $Q \simeq 0.01\left(\Lambda / 3 \times 10^{-14} \mathrm{~s}^{-1}\right)(T / 10 \mathrm{~K})^{1 / 2}\left(M_{\mathrm{cbd}} / 0.4 M_{\odot}\right)^{-1}\left(\mathcal{A}_{\mathrm{cbd}} / 4 \times\right.$ $\left.10^{33} \mathrm{~cm}^{2}\right)$. Considering the uncertainties in the input parameters, it is certain that the $Q$-value is less than unity. We therefore suggest that the binary system is likely to be forming in the core center due to the gravitational fragmentation of the circumbinary disk-like structure, whose radius appears to be determined by the rotation of the core.

\section{SUMMARY AND FUTURE WORK}

The main findings of this work and future considerations are summarized as follows:

1. Our high-density gas observations identified a molecular cloud core with a size of $\simeq 0.08$ pc toward GF 9-2. In the core center, a protostellar envelope with a size of $\simeq 4500 \mathrm{AU}$ is identified through $350 \mu \mathrm{m}$ continuum emission. The masses of the core and envelope are $\simeq 3$ and $\simeq 0.6 M_{\odot}$, respectively. Obtaining fractional abundances of the probe molecules, we propose that the GF 9-2 core should be at an early phase of chemical evolution, although the radial variations of the abundances should be revealed to accurately assess the chemical evolutionary stage.
2. Our deep search for CO wing emission revealed that the protostar in GF 9-2 has not developed a large-scale high-velocity "jetlike" outflow. Instead, a compact low-velocity outflow may be formed at the core center. Thus, GF 9-2 is very likely to be at an extremely early stage in low-mass star formation before arriving at its most active outflow phase. To test the conclusion, we need to conduct a high angular resolution and deeper search for compact outflow(s) in higher $J$ transitions of CO.

3. The radial column density profile of the core can be well fitted by a power-law form of $\rho(r) \propto r^{-2}$ for the $0.003<r /$ pc $<$ 0.08 region. The power-law index of -2 agrees with the expectation for an outer part of the gravitationally collapsing core. On the other hand, based on the absence of a $\rho(r) \propto r^{-3 / 2}$ profile, we found that a central free-fall region has not developed in a region larger than $r \simeq 600 \mathrm{AU}$.

4. The PV analysis identified a large-scale velocity gradient with an order of $1 \mathrm{~km} \mathrm{~s}^{-1} \mathrm{pc}^{-1}$ along the major axis of the core. The gradient is interpreted as a rigid-body-like rotation with an angular velocity of $\simeq 3 \times 10^{-14} \mathrm{~s}^{-1}$, comparable to those measured in other similar cores. We found that the gas is supersonic all over the core and that the velocity width is enhanced up to $\simeq 0.6 \mathrm{~km} \mathrm{~s}^{-1}$ in the central 1000 AU toward the $3 \mathrm{~mm}$ source. The supersonic velocity width and the $\rho(r) \propto r^{-2}$ profile are explained by infall in the extension of the runaway collapse scenario proposed by Larson and Penston for $t>0$. The current mass accretion rate of $\simeq 3 \times 10^{-5} M_{\odot} \mathrm{yr}^{-1}$ is estimated from $v_{\text {inf }} \simeq 0.3 \mathrm{~km} \mathrm{~s}^{-1}$ at $r \simeq 7000$ AU. We discuss the likelihood that the GF 9-2 core has been undergoing gravitational collapse $\lesssim 5000 \mathrm{yr}$, starting from initially unstable conditions.

5. We discovered a potential protobinary system with a projected separation of $\simeq 1200$ AU embedded in a circumbinary disk-like structure with $\simeq 2000 \mathrm{AU}$ radius at the core center. The binary consists of a very young protostar and a preprotostellar condensation. The circumbinary material mass is on the order of $0.1 M_{\odot}$. Taking account of the centrifugal radius calculated from the core rotation and the Toomre $Q$-value for the disk, we suggest that the binary formation may have been initiated by gravitational fragmentation of the circumbinary disk-like structure. Further studies are needed using high angular resolution deep imaging in submillimeter and far-infrared continuum emission.

During the final refereeing process, we have detected clear blue-skewed spectra in the $\mathrm{HCO}^{+}(3-2)$ line using the CSO $10.4 \mathrm{~m}$ telescope, supporting our interpretation of infall in $\S 5.2$.

The authors sincerely acknowledge an anonymous referee whose comments significantly improved the quality of the paper. R. S. F. acknowledges A. I. Sargent and J. M. Carpenter for critical readings of the manuscript and continuous encouragement. The authors thank H. Yoshida for providing the telescope time at CSO to obtain the ${ }^{12} \mathrm{CO}(3-2)$ data, S. Takakuwa, J. Lamb, D. Woody, K. Sunada, and S. Okumura for fruitful discussion about data combining of the single-dish telescope and interferometer, H. Wiesemeyer for information on GF 9-2 including the distance and the luminosity, and L. Testi for his early contribution to this study. R. S. F. also appreciates discussion with A. Wootten, R. Cesaroni, E. van Dishoeck, H. Butner, G. Blake, T. Hanawa, T. Matsumoto, Y. Aikawa, K. Tachihara, T. Velusamy, and D. Ward-Thompson. R. S. F. and H. S. thank M. Yang, A. Kovács, C. Borys, and A. C. A. Boogert for their support at the SHARC II observations and data reduction. The authors gratefully acknowledge 
all of the staff at OVRO, NRO, CSO, VLA, and the GILDAS software group at IRAM. Research at the Owens Valley Radio Obser- vatory is supported by the National Science Foundation through NSF grant AST 02-28955.

\section{APPENDIX A}

\section{COMBINING THE OVRO MILLIMETER ARRAY AND NOBEYAMA $45 \mathrm{~m}$ TELESCOPE DATA}

We describe a method of combining the OVRO millimeter array and Nobeyama $45 \mathrm{~m}$ telescope data to obtain high spatial resolution and missing flux-free images. We used the $45 \mathrm{~m}$ data to fill the central $u-v$ hole of the millimeter array visibility data. Since the OVRO array consists of six $10.4 \mathrm{~m}$ diameter telescopes, we can attain projected baseline length normalized by the observing wavelength $\left(D_{\lambda}\right)$ of $\geq 3.5 \mathrm{k} \lambda$ at $3 \mathrm{~mm}$ when the array takes the most compact " $\mathrm{C}$ " configuration. This minimum spatial frequency is sufficiently smaller than the maximum spatial frequency of $D_{\lambda}=15 \mathrm{k} \lambda$ for a spatially Nyquist-sampled $45 \mathrm{~m}$ telescope map. Therefore, the usage of the $45 \mathrm{~m}$ maps works to recover the flux densities missed by the millimeter array. The basics of the data combination are described in Vogel et al. (1984), and our method here is similar to those in the previous works (e.g., Takakuwa et al. 2003). We took the following steps to make the combined maps:

1. For the BEARS $\mathrm{H}^{13} \mathrm{CO}^{+}$data, we used the spectra taken with the inner nine beams centered on the central beam on-axis. The alignment of the nine beams can be approximated as a regular grid with 41 ". 4 spacing for our rms pointing accuracy of $\leqslant 2^{\prime \prime}$. This makes the resultant map grid spacing of 20 "' 7 , i.e., full-beam sampling. Obtaining the data on a regular grid is required to use FFT. As for the $\mathrm{N}_{2} \mathrm{H}^{+}$, we used the data on a regular grid of $17^{\prime \prime}$ spacing (full-beam sampling) with pointing accuracy of $\lesssim 2^{\prime \prime}$. To achieve the excellent pointing accuracy, we used the spectra under wind speeds of $\lesssim 2 \mathrm{~m} \mathrm{~s}^{-1}$. We corrected the $T_{\mathrm{mb}}$ scale of the $\mathrm{H}^{13} \mathrm{CO}^{+}$data with the BEARS in double-sideband mode by observing the core center with the $\mathrm{S} 80 / \mathrm{S} 100$ receivers having single sideband filters. Since $T_{\mathrm{mb}}$ at the core center is $0.73 \pm 0.04 \mathrm{~K}$ by the $\mathrm{S} 80 / \mathrm{S} 100$ receivers with an 18.5 beam and $0.91 \pm 0.1 \mathrm{~K}$ by the BEARS with a $20^{\prime \prime} 3$ beam, we scaled the intensities of the BEARS data by a factor of 0.80 .

2. To have a common frequency resolution between the OVRO and $45 \mathrm{~m}$ telescope data, the $45 \mathrm{~m} \mathrm{~N}_{2} \mathrm{H}^{+}$spectra, which have a $37 \mathrm{kHz}$ spectral resolution, are smoothed in the same frequency bins as those of the OVRO data with a $125 \mathrm{kHz}$ resolution. For the $\mathrm{H}^{13} \mathrm{CO}^{+}$ line, the $45 \mathrm{~m}$ and OVRO data have the same frequency bins.

3. We made the $45 \mathrm{~m}$ maps by full-beam sampling on the regular grids with spacings of $20^{\prime \prime} .7\left(=41\right.$ ".4/2) for $\mathrm{H}^{13} \mathrm{CO}^{+}$and $17^{\prime \prime} .0$ for $\mathrm{N}_{2} \mathrm{H}^{+}$. We did not apply the task BPOSC in the NEWSTAR package, which corrects the misalignment between the 25 beams on the sky plane, to the $\mathrm{H}^{13} \mathrm{CO}^{+}$data. Note that our OVRO field of view (FOV) with a single pointing does not cover the whole extent of the core.

4. In converting the $T_{\mathrm{mb}}$ scale of the $45 \mathrm{~m}$ maps into the flux density per beam solid angle $\left(F_{\nu}\right)$, we calculated conversion factors through a relation of $F_{\nu}=I_{\nu} \Omega_{\text {beam }}$ with the Rayleigh-Jeans approximation. Here $I_{\nu}$ is the specific intensity and $\Omega_{\text {beam }}$ is the beam solid angle. The conversion factors are given by $\left(F_{\nu} / T_{\mathrm{mb}}\right)=\left(2 k / \lambda^{2}\right)\left(\pi \theta_{\mathrm{HPBW}}^{2} / 4 \ln 2\right)=3.27(3 \mathrm{~mm} / \lambda)^{2}\left(\theta_{\mathrm{HPBW}} / 20^{\prime \prime}\right)^{2} \mathrm{Jy} \mathrm{beam}^{-1} \mathrm{~K}^{-1}$.

5. To deconvolve the $45 \mathrm{~m}$ maps with its beam, the beam pattern is approximated by an axisymmetric Gaussian profile with HPBW of 20.3 at $86.754 \mathrm{GHz}$ for the BEARS $\mathrm{H}^{13} \mathrm{CO}^{+}$data and $17^{\prime \prime 2} 2$ at $93.173 \mathrm{GHz}$ for the $\mathrm{S} 80 / \mathrm{S} 100 \mathrm{~N}_{2} \mathrm{H}^{+}$data. We adopt a mean HPBW of the inner nine beams for BEARS $\left(20^{\prime \prime} 3 \pm 0.3\right) \mathrm{H}^{13} \mathrm{CO}^{+}$observations. Note that the S80/S100 receivers have nearly equal beam pattern as they detect two independent linearly polarized waves by sharing common dewar and waveguide.
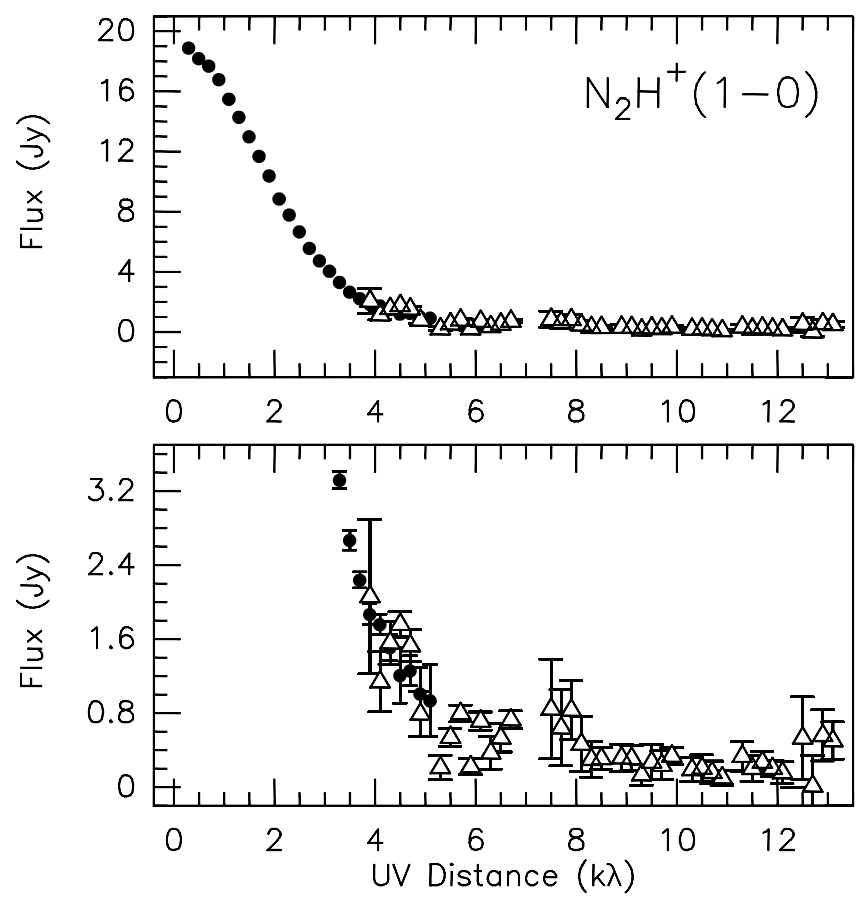

FIG. 16.-Plots of flux density in Jy vs. projected baseline length in $\mathrm{k} \lambda$ for the $\mathrm{N}_{2} \mathrm{H}^{+}(1-0)$ line data with the $45 \mathrm{~m}$ telescope ( filled circles) and the OVRO millimeter array (open triangles). The data are from the $V_{\mathrm{LSR}}=-2.6 \mathrm{~km} \mathrm{~s}^{-1}$ channel of the strongest $\mathrm{HF}$ component of the $1_{23}-0_{12}$ transition. The bottom panel is a magnified plot of the top one. The visibilities in the plots are resampled with 0.2 and $0.4 \mathrm{k} \lambda$ bins for the $45 \mathrm{~m}$ and OVRO data, respectively. 


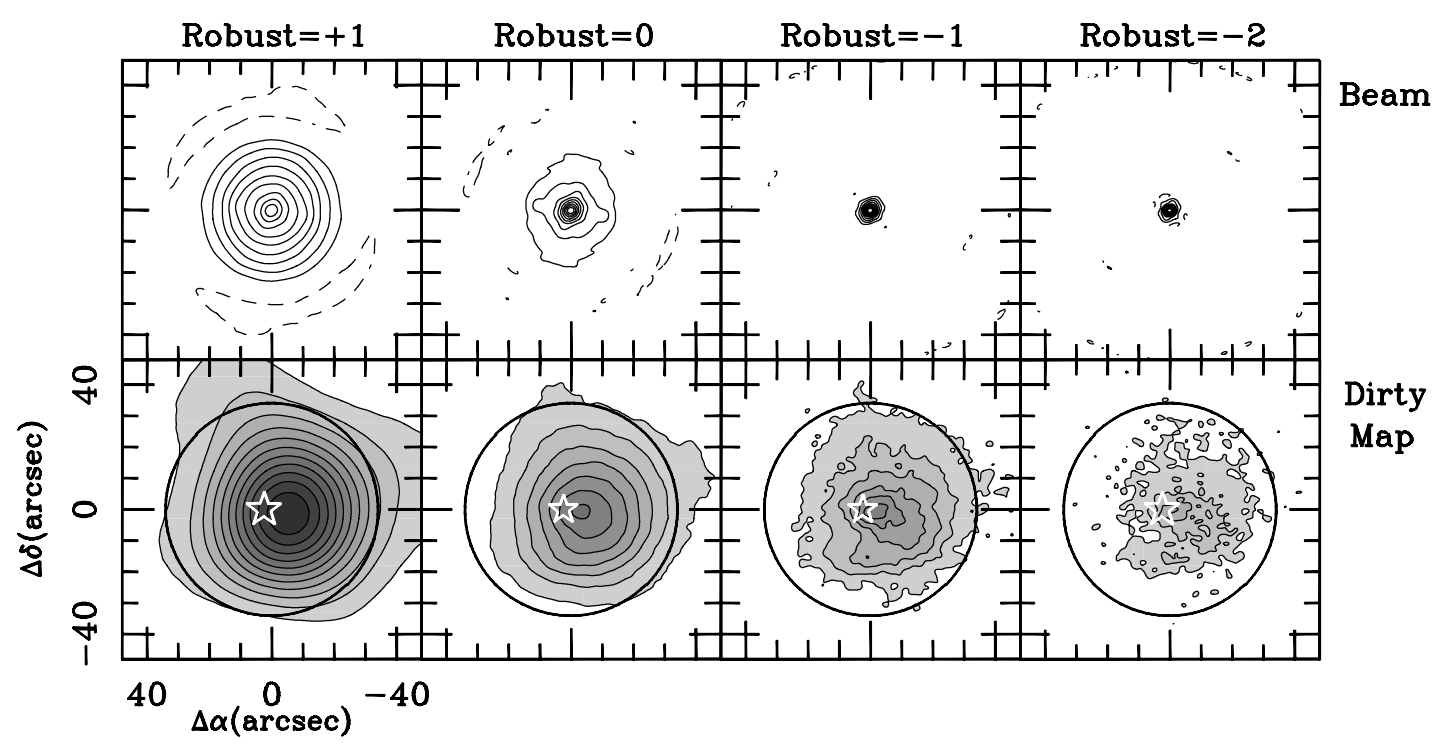

FIG. 17.-Beam patterns (top) and "dirty maps" (bottom) of the combined $\mathrm{N}_{2} \mathrm{H}^{+}(1-0)$ data for the robustness powers of $+1,0,-1$, and -2 . The data are from the $-2.6 \mathrm{~km} \mathrm{~s}^{-1}$ channel map of the strongest $\mathrm{HF}$ component of the $1_{23}-0_{12}$ transition. Contour intervals for the beams are $10 \%$ of the peak values, starting from the $\pm 10 \%$ levels. Dashed contours are complementary negative contours. Contours in the dirty maps have $3 \sigma$ steps, starting from the $3 \sigma$ levels; the $1 \sigma$ noise levels are 127.5, 82.4, 28.5, and $24.7 \mathrm{mJy}_{\text {beam }}^{-1}$ for the robustness powers of $+1,0,-1$, and -2 , respectively. The synthesized beam sizes of $\theta_{\text {maj }} \times \theta_{\min }$ are $10^{\prime \prime} 33 \times 99^{\prime \prime} 18,5^{\prime \prime} 81 \times 5^{\prime \prime} .09,4{ }^{\prime \prime} .16 \times 33^{\prime \prime} 57$, and 3 ."27 $\times 2$ "' 66 for the robustness powers of $+1,0,-1$, and -2 , respectively. The central stars in the bottom panels represent the position of the $3 \mathrm{~mm}$ source.

6. The $45 \mathrm{~m}$ maps are deconvolved by the Gaussian beams defined in step 5 . Since our $45 \mathrm{~m}$ maps satisfy the criteria discussed in Vogel et al. (1984), the deconvolution is done by dividing the maps by the beam pattern. After several trials including the steps described below, the most plausible combined maps are obtained when the cutoff level of deconvolution is set to be $20 \%$ of the rms noise level of the single-dish map so as not to miss high spatial frequency components having low amplitudes.

7. The deconvolved $45 \mathrm{~m}$ maps are convolved with the primary beam pattern of the OVRO $10.4 \mathrm{~m}$ antennas, assuming that all six element telescopes have an axisymmetric Gaussian profile with HPBWs of $699^{\prime \prime} 7$ at $93.174 \mathrm{GHz}$ and $75^{\prime \prime} 0$ at $86.754 \mathrm{GHz}$.

8. The single-dish maps are converted into visibility data in the $u-v$ domain by FFT. As demonstrated in Figure 16, the visibility amplitudes from the two instruments agree well with each other over the common $u$ - $v$ distances. We stress that we did not apply amplitude scale corrections to both the $45 \mathrm{~m}$ and OVRO data.

9. The FFT single-dish visibilities are used to make model visibilities that have the same integration time as the OVRO data (205 s). Since our $45 \mathrm{~m}$ maps are sampled with the full-beam spacing, $\theta_{f}$, we made model visibilities between zero and $D_{\lambda}=1 /\left(2 \theta_{f}\right)$ on the basis of the sampling theorem. We calculated $D_{\lambda}=6.10$ and $5.08 \mathrm{k} \lambda$ for the $\mathrm{N}_{2} \mathrm{H}^{+}$and $\mathrm{H}^{13} \mathrm{CO}^{+}$data, respectively, and conservatively adopted smaller cutoff values of 5.3 and $4.5 \mathrm{k} \lambda$ to fill the central $u-v$ holes in the OVRO data (Table 3).

10. We combined the $45 \mathrm{~m}$ and OVRO visibilities in the $u$-v plane, assuming that the $45 \mathrm{~m}$ model visibilities have a Gaussian distribution within a radius of $D_{\lambda}$ adopted in item 9 . We found that synthesized beams of the combined maps are controlled by the visibility weighting function, regardless of the distribution of single-dish model visibilities.

11. To construct an image from the combined visibilities, we used the task IMAGR in the AIPS package where one can optimize the synthesized beam size and sensitivity of the resultant image by changing the robustness power of the weighting function. Figure 17 represents how the parameter affects the synthesized beam pattern and the dirty map (map affected by the sidelobe pattern of the synthesized beam). Considering the trade-off between the beam size and the smoothness of the map, we selected the robustness power of -1 and CLEANed the dirty maps. We stopped the CLEAN loop before encountering the first negative CLEAN component because we are unable to find emission-free regions to estimate an rms noise level, i.e., a threshold flux density to stop the loop, due to the larger extent of the core than the OVRO FOV.

\section{APPENDIX B}

\section{CALCULATION OF COLUMN DENSITY}

To estimate the total column density of molecular gas $\left(N_{\text {tot }}\right)$ through observations of the rotational transition from the upper $J+1$ to lower $J$ level, we use the previously derived equation (e.g., Scoville et al. 1986) that gives $N_{\text {tot }}$ through the optical depth $\left(\tau_{v}\right)$ and excitation temperature $\left(T_{\mathrm{ex}}\right)$ of the probe molecule as follows:

$$
N_{\text {tot }}=\frac{3 k}{8 \pi^{3} \mu^{2} B} \frac{\left(T_{\mathrm{ex}}+h B / 3 k\right)}{J+1} \frac{\exp \left[h B J(J+1) / k T_{\mathrm{ex}}\right]}{1-\exp \left(-h \nu / k T_{\mathrm{ex}}\right)} \int \tau_{v} d v,
$$

where $k$ denotes Boltzmann's constant, $h$ is Planck's constant, $\mu$ is the permanent dipole moment of the molecule, and $B$ is the rotational constant. We should keep in mind that to derive equation (B1), one has approximated the rotational partition function $Q\left(T_{\mathrm{ex}}\right)$ as

$$
Q\left(T_{\mathrm{ex}}\right) \simeq \frac{k}{h B}\left(T_{\mathrm{ex}}+\frac{h B}{3 k}\right) .
$$




\section{B1. $\mathrm{N}_{2} \mathrm{H}^{+}$}

To apply equation (B1) to the $\mathrm{N}_{2} \mathrm{H}^{+}$line data, we have to find $T_{\mathrm{ex}}$ and $\tau_{\nu}$. In the following, we describe how they are obtained from the $\mathrm{N}_{2} \mathrm{H}^{+}$spectra through the HFS analysis. In LTE, an observed brightness temperature $\left(T_{B}\right)$ is expressed by the following radiative transfer equation:

$$
T_{b}=f\left[J_{\nu}\left(T_{\mathrm{ex}}\right)-J_{\nu}\left(T_{\mathrm{bg}}\right)\right]\left(1-e^{-\tau_{\nu}}\right),
$$

where $T_{B}$ is replaced by the brightness temperatures averaged over a main beam $\left(T_{\mathrm{mb}}\right)$ for single-dish observations and over a synthesized beam $\left(T_{\mathrm{sb}}\right)$ for interferometric observations. Here $f$ denotes a beam filling factor and $J_{\nu}(T)$ is the equivalent RayleighJeans temperature given by $J_{\nu}(T)=(h \nu / k)[\exp (h \nu / k T)-1]^{-1}$. From equation (B3), we fit the function of $y(v)=C\{1-$ $\exp [-\tau(v)]\}$ with a constant $C$ to each $\mathrm{N}_{2} \mathrm{H}^{+} \mathrm{HF}$ component. The optical depth is given by

$$
\tau(v)=\tau_{\text {tot }} \sum_{i=1}^{7} s_{i} \exp \left\{-\frac{\left[v-\left(V_{\text {sys }}+\delta v_{i}\right)\right]^{2}}{2 \sigma_{i}^{2}}\right\},
$$

as a function of velocity. Here $\tau_{\text {tot }}$ denotes the total optical depth, i.e., a sum of the optical depths at the centers of the HF components; $s_{i}$ is the normalized relative intensity of the $i$ th HF component taken from Cazzoli et al. (1985); $\delta v_{i}$ is the velocity offset of the $i$ th component; and $\sigma_{i}^{2}=\left(\Delta v_{\mathrm{FWHM}, i}\right)^{2} / 8 \ln 2$ with the velocity width in FWHM of the $i$ th component $\left(\Delta v_{\mathrm{FWHM}, i}\right)$. We assumed that the line broadenings due to the thermal and nonthermal gas motions equally work for all of the HF components since they are emanated from the same molecular ion in the same region. We thus simplified equation (B4) by adopting $\sigma_{i}=\sigma$ and $\Delta v_{\mathrm{FWHM}, i}=\Delta v_{\mathrm{FWHM}}$. Therefore, the HFS analysis gives $\tau_{\text {tot }}$, the center velocity $\left(V_{\text {sys }}+\delta v_{i}\right)$, the velocity width $\left(\Delta v_{\mathrm{FWHM}}\right)$, and the constant $C$. Since $C=f\left[J_{\nu}\left(T_{\mathrm{ex}}\right)-J_{\nu}\left(T_{\mathrm{bg}}\right)\right]$, one can calculate $T_{\mathrm{ex}}$ by

$$
T_{\mathrm{ex}}=\frac{h \nu_{0}}{k}\left(\ln \left\{1+\frac{h \nu_{0}}{k\left[C+J_{\nu}\left(T_{\mathrm{bg}}\right)\right]}\right\}\right)^{-1} .
$$

Here we assumed $f=1.0$ because the region of our interest is larger than the beam sizes. We now have the estimates of $T_{\mathrm{ex}}, \tau_{\text {tot }}$, and $\Delta v_{\text {int }}$ at each observing point (each pixel for the combined data). Since the integral in equation (B1) becomes

$$
\int \tau_{v} d v=\frac{1}{2} \sqrt{\frac{\pi}{\ln 2}} \sum_{i=1}^{7} \tau_{i} \Delta v_{\text {int }}=\frac{1}{2} \sqrt{\frac{\pi}{\ln 2}} \tau_{\text {tot }} \Delta v_{\text {int }},
$$

we have the desired expression to calculate the column density of $\mathrm{N}_{2} \mathrm{H}^{+}\left(N_{\mathrm{N}_{2} \mathrm{H}^{+}}\right)$as follows:

$$
N_{\mathrm{N}_{2} \mathrm{H}^{+}}=3.30 \times 10^{11} \frac{\left(T_{\mathrm{ex}}+0.75\right)}{1-e^{-4.47 / T_{\mathrm{ex}}}}\left(\frac{\tau_{\text {tot }}}{1.0}\right)\left(\frac{\Delta v_{\text {int }}}{1.0 \mathrm{~km} \mathrm{~s}^{-1}}\right) \mathrm{cm}^{-2} .
$$

Here we used $\mu=3.4 \mathrm{D}$ (Havenith et al. 1990) and $B=46586.702 \mathrm{MHz}$ (Caselli et al. 1995).

\section{B2. $\mathrm{H}^{13} \mathrm{CO}^{+}$}

We estimated the column density of $\mathrm{H}^{13} \mathrm{CO}^{+}\left(N_{\mathrm{H}^{13} \mathrm{CO}^{+}}\right)$with equation (B1) giving the mean $T_{\mathrm{ex}}$ and $\tau_{\nu_{0}}$ over the full extent of the core (Table 5) where $\tau_{\nu_{0}}$ is a mean optical depth at the line center frequency of $\nu_{0}$. Since the radiative transfer equation of equation (B3) is written as $T_{b}=f\left[J_{\nu}\left(T_{\mathrm{ex}}\right)-J_{\nu}\left(T_{\mathrm{bg}}\right)\right] \tau_{\nu}\left[\left(1-e^{-\tau_{\nu}}\right) / \tau_{\nu}\right]$, the integral of $\tau_{\nu}$ can be expressed as

$$
\int \tau_{\nu}(v) d v \simeq \frac{\tau_{\nu_{0}}}{1-e^{-\tau_{\nu_{0}}}} \frac{\int T_{b}(v) d v}{J_{\nu}\left(T_{\mathrm{ex}}\right)-J_{\nu}\left(T_{\mathrm{bg}}\right)}
$$

for $\tau_{\nu} \simeq 0$.

Recall that $\mathrm{H}^{13} \mathrm{CO}^{+}(1-0)$ in GF 9-2 is optically thin (§ 4.1.2) and we assumed $f=1$ in $\S \mathrm{B} 1$. Combining equations (B1) and (B8), we obtain an expression to estimate the $\mathrm{H}^{13} \mathrm{CO}^{+}$column density from single-dish data as

$$
N_{\mathrm{H}^{13} \mathrm{CO}^{+}}=2.32 \times 10^{11} \frac{\left(T_{\mathrm{ex}}+0.69\right)}{1-e^{-4.16 / T_{\mathrm{ex}}}} \frac{1}{J_{\nu}\left(T_{\mathrm{ex}}\right)-J_{\nu}\left(T_{\mathrm{bg}}\right)}\left(\frac{\int T_{\mathrm{mb}} d v}{1.0 \mathrm{~K} \mathrm{~km} \mathrm{~s}^{-1}}\right) \mathrm{cm}^{-2} .
$$

Here we took $\mu=4.07 \mathrm{D}$ (Haese \& Woods 1979 ) and $B=43377.17 \mathrm{MHz}$. To have a complementary expression for interferometric data, we converted the flux density $\left(F_{\nu}\right)$ per synthesized beam into a brightness temperature per beam $\left(T_{\mathrm{sb}}\right)$ through $T_{\mathrm{sb}}=\left(\lambda^{2} / 2 k \Omega_{b}\right) F_{\nu}$, where $\Omega_{b}$ is a beam solid angle given by $(\pi / 4 \ln 2)\left(\theta_{\text {maj }} \times \theta_{\text {min }}\right)$. Therefore, we have

$$
N_{\mathrm{H}^{13} \mathrm{CO}^{+}}=3.77 \times 10^{13} \frac{\left(T_{\mathrm{ex}}+0.69\right)}{1-e^{-4.16 / T_{\mathrm{ex}}}} \frac{1}{J_{\nu}\left(T_{\mathrm{ex}}\right)-J_{\nu}\left(T_{\mathrm{bg}}\right)}\left(\frac{\Omega_{b}}{1^{\prime \prime} \times 1^{\prime \prime}}\right)^{-1}\left(\frac{\int F_{\nu} d v}{1.0 \mathrm{Jy} \mathrm{km} \mathrm{s}^{-1}}\right) \mathrm{cm}^{-2} .
$$




\section{B3. CCS}

Column density analysis of the CCS $4_{3}-3_{2}$ line is similar to that for the $\mathrm{H}^{13} \mathrm{CO}^{+}$line in the sense that we have only the representative values of $T_{\mathrm{ex}}$ and $\tau_{\nu_{0}}$ over the entire core (Table 5). The observed CCS brightness temperatures are converted into the column density through

$$
N_{\mathrm{CCS}}=8.16 \times 10^{11} \frac{\left(T_{\mathrm{ex}}+0.10\right) \exp \left(3.73 / T_{\mathrm{ex}}\right)}{1-e^{-2.18 / T_{\mathrm{ex}}}} \frac{\tau_{\nu_{0}}}{1-e^{-\tau_{\nu_{0}}}} \frac{1}{J_{\nu}\left(T_{\mathrm{ex}}\right)-J_{\nu}\left(T_{\mathrm{bg}}\right)}\left(\frac{\int T_{\mathrm{mb}} d v}{1.0 \mathrm{~K} \mathrm{~km} \mathrm{~s}^{-1}}\right) \mathrm{cm}^{-2} .
$$

Here we used $\mu=2.81 \mathrm{D}$ (Murakami 1990) and $B=6477.75036 \mathrm{MHz}$ (Yamamoto et al. 1990). Although the rotational energy $\left(E_{r}\right)$ of the CCS molecule is given by $E_{r}=h B J(J+1)-h D J^{2}(J+1)^{2}$ considering the centrifugal stretching with $D=1727.96 \mathrm{kHz}$ (Yamamoto et al. 1990), we ignored the $D$ term to use the rotational partition function of equation (B2).

\section{REFERENCES}

Aikawa, Y., Ohashi, N., Inutsuka, S., Herbst, E., \& Takakuwa, S. 2001, ApJ 552,639

André, P., Martin-Pintado, J., Despois, D., \& Montmerle, T. 1990, A\&A, 236, 180

André, P., Motte, F., \& Bacmann, A. 1999, ApJ, 513, L57

André, P., Ward-Thompson, D., \& Barsony, M. 1993, ApJ, 406, 122 2000, in Protostars and Planets IV, ed. V. Mannings, A. P. Boss, \& S. S. Russell (Tucson: Univ. Arizona Press), 59

André, P., Ward-Thompson, D., \& Motte, F. 1996, A\&A, 314, 625

Bachiller, R. 1996, ARA\&A, 34, 111

Beckwith, S. V. W., Henning, T., \& Nakagawa, Y. 2000, in Protostars and Planets IV, ed. V. Mannings, A. P. Boss, \& S. S. Russell (Tucson: Univ. Arizona Press), 533

Belloche, A., \& André, P. 2004, A\&A, 419, L35

Benson, P. J., Caselli, P., \& Myers, P. C. 1998, ApJ, 506, 743

Bergin, E. A., \& Langer, W. D. 1997, ApJ, 486, 316

Butner, H. M., Lada, E. A., \& Loren, R. B. 1995, ApJ, 448, 207

Cabrit, S., \& Bertout, C. 1992, A\&A, 261, 274

Caselli, P., Benson, P. J., Myers, P. C., \& Tafalla, M. 2002, ApJ, 572, 238

Caselli, P., Myers, P. C., \& Thaddeus, P. 1995, ApJ, 455, L77

Cazzoli, G., Corbelli, G., Degli Esposti, C., \& Favero, P. G. 1985, Chem. Phys. Lett., 118, 164

Chen, H., Myers, P. C., Ladd, E. F., \& Wood, D. O. S. 1995, ApJ, 445, 377

Chernin, L. M. 1995, ApJ, 440, L97

Claussen, M. J., Marvel, K. B., Wootten, A., \& Wilking, B. A. 1998, ApJ, 507, L79

Codella, C., Bachiller, R., \& Reipurth, B. 1999, A\&A, 343, 585

Cox, P., Walmsley, C. M., \& Güsten, R. 1989, A\&A, 209, 382

Dickman, R. L. 1978, ApJS, 37, 407

Dowell, C. D., et al. 2003, Proc. SPIE, 4855, 73

Foster, P. N., \& Chevalier, R. A. 1993, ApJ, 416, 303

Furuya, R. S., Kitamura, Y., Saito, M., Kawabe, R., \& Wootten, H. A. 1999, ApJ, 525, 821

Furuya, R. S., Kitamura, Y., Wootten, H. A., Claussen, M. J., \& Kawabe, R. 2001, ApJ, 559, L143 2003, ApJS, 144, 71

Furuya, R. S., Kitamura, Y., Wootten, H. A., Claussen, M. J., Saito, M., Marvel, K. B., \& Kawabe, R. 2000, ApJ, 542, L135

Goodman, A. A., Benson, P. J., Fuller, G. A., \& Myers, P. C. 1993, ApJ, 406, 528

Haese, N. N., \& Woods, R. C. 1979, Chem. Phys. Lett., 61, 396

Havenith, M., Zwart, E., Leo Meerts, W., \& Ter Meulen, J. J. 1990, J. Chem. Phys., 93, 8446

Ho, P. T. P., \& Townes, C. H. 1983, ARA\&A, 21, 239

Hunter, C. 1977, ApJ, 218, 834

Jijina, J., Myers, P. C., \& Adams, F. C. 1999, ApJS, 125, 161

Kovács, A. 2006, Ph.D. thesis, Caltech

Kuiper, T. B. H., Langer, W. D., \& Velusamy, T. 1996, ApJ, 468, 761

Lai, S.-P., \& Crutcher, R. M. 2000, ApJS, 128, 271

Larson, R. B. 1969, MNRAS, 145, 271

Leong, M. M. 2005, URSI Conf. Sec. J3-10, 426

Mizuno, A., Onishi, T., Hayashi, M., Ohashi, N., Sunada, K., Hasegawa, T., \& Fukui, Y. 1994, Nature, 368, 719

Moriarty-Schieven, G. H., Wannier, P. G., Keene, J., \& Tamura, M. 1994, ApJ, 436, 800
Moscadelli, L., Testi, L., Furuya, R. S., Goddi, C., Claussen, M., Kitamura, Y., \& Wootten, A. 2006, A\&A, 446, 985

Mundy, L. G., Looney, L. W., \& Welch, W. J. 2000, in Protostars and Planets IV, ed. V. Mannings, A. P. Boss, \& S. S. Russell (Tucson: Univ. Arizona Press), 355

Murakami, A. 1990, ApJ, 357, 288

Myers, P. C., \& Benson, P. J. 1983, ApJ, 266, 309

Myers, P. C., \& Ladd, E. F. 1993, ApJ, 413, L47

Ogino, S., Tomisaka, K., \& Nakamura, F. 1999, PASJ, 51, 637

Ossenkopf, V., \& Henning, T. 1994, A\&A, 291, 943

Penston, M. V. 1969, MNRAS, 144, 425

Phillips, T. G., Wannier, P. G., Scoville, N. Z., \& Huggins, P. J. 1979, ApJ, 231, 720

Preibisch, T., Ossenkopf, V., Yorke, H. W., \& Henning, T. 1993, A\&A, 279, 577

Richer, J. S., Padman, R., Ward-Thompson, D., Hills, R. E., \& Harris, A. I. 1993, MNRAS, 262, 839

Richer, J. S., Shepherd, D. S., Cabrit, S., Bachiller, R., \& Churchwell, E. 2000, in Protostars and Planets IV, ed. V. Mannings, A. P. Boss, \& S. S. Russell (Tucson: Univ. Arizona Press), 867

Schilke, P., Walmsley, C. M., Pineau des Forets, G., \& Flower, D. R. 1997, A\&A, 321, 293

Schneider, S., \& Elmegreen, B. G. 1979, ApJS, 41, 87

Scoville, N. Z., Sargent, A. I., Sanders, D. B., Claussen, M. J., Masson, C. R., Lo, K. Y., \& Phillips, T. G. 1986, ApJ, 303, 416

Shinnaga, H., Ohashi, N., Lee, S., \& Moriarty-Schieven, G. H. 2004, ApJ, 601, 962

Shu, F. H. 1977, ApJ, 214, 488

Shu, F. H., Adams, F. C., \& Lizano, S. 1987, ARA\&A, 25, 23

Stutzki, J., Ungerechts, H., \& Winnewisser, G. 1982, A\&A, 111, 201

Suzuki, H., Yamamoto, S., Ohishi, M., Kaifu, N., Ishikawa, S., Hirahara, Y., \& Takano, S. 1992, ApJ, 392, 551

Tafalla, M., Myers, P. C., Caselli, P., \& Walmsley, C. M. 2004, A\&A, 416, 191

Tafalla, M., Myers, P. C., Caselli, P., Walmsley, C. M., \& Comito, C. 2002, ApJ, 569, 815

Takakuwa, S., Kamazaki, T., Saito, M., \& Hirano, N. 2003, ApJ, 584, 818

Terebey, S., Vogel, S. N., \& Myers, P. C. 1992, ApJ, 390, 181

Ungerechts, H., Walmsley, C. M., \& Winnewisser, G. 1980, A\&A, 88, 259

Vogel, S. N., Wright, M. C. H., Plambeck, R. L., \& Welch, W. J. 1984, ApJ, 283,655

Walker, C. K., Lada, C. J., Young, E. T., Maloney, P. R., \& Wilking, B. A. 1986, ApJ, 309, L47

Ward-Thompson, D., Scott, P. F., Hills, R. E., \& Andre, P. 1994, MNRAS, 268, 276

Whitworth, A., \& Summers, D. 1985, MNRAS, 214, 1

Wiesemeyer, H. 1997, Ph.D. thesis, Univ. Bonn

Wiesemeyer, H., Cox, P., Güsten, R., \& Zylka, R. 1999, in The Universe as Seen by ISO, ed. P. Cox \& M. F. Kessler (ESA SP-427; Noordwijk: ESA), 533

Winnewisser, G., Churchwell, E., \& Walmsley, C. M. 1979, A\&A, 72, 215

Wolkovitch, D., Langer, W. D., Goldsmith, P. F., \& Heyer, M. 1997, ApJ, 477, 241

Womack, M., Ziurys, L. M., \& Wyckoff, S. 1992, ApJ, 387, 417

Yamamoto, S., et al. 1990, ApJ, 361, 318

Zhou, S. 1992, ApJ, 394, 204 